Portland State University

PDXScholar

8-4-2021

\title{
A Multiplicity of Journeys: STEM Education Ecosystems as Sources of Cultural Sustenance
}

Christopher Louis Beauprey Cardiel

Portland State University

Follow this and additional works at: https://pdxscholar.library.pdx.edu/open_access_etds

Part of the Curriculum and Instruction Commons, and the Science and Mathematics Education Commons

Let us know how access to this document benefits you.

\section{Recommended Citation}

Cardiel, Christopher Louis Beauprey, "A Multiplicity of Journeys: STEM Education Ecosystems as Sources of Cultural Sustenance" (2021). Dissertations and Theses. Paper 5759.

https://doi.org/10.15760/etd.7630

This Dissertation is brought to you for free and open access. It has been accepted for inclusion in Dissertations and Theses by an authorized administrator of PDXScholar. Please contact us if we can make this document more accessible: pdxscholar@pdx.edu. 
A Multiplicity of Journeys:

STEM Education Ecosystems as Sources of Cultural Sustenance

\author{
by \\ Christopher Louis Beauprey Cardiel
}

A dissertation submitted in partial fulfillment of the requirements for the degree of

Doctor of Education

in

Educational Leadership: Curriculum and Instruction

Dissertation Committee:

Anita Bright, Chair

Micki M. Caskey

Amanda Sugimoto

Alma Trinidad

Portland State University

2021 
(C) 2021 Christopher Louis Beauprey Cardiel 


\begin{abstract}
In this document, I outline the context and significance of a research problem faced by both formal and informal science, technology, engineering, and math (STEM) educators in the Portland metro area-specifically, the need for greater understanding of the individual and cultural motivations, needs, and agentic behavior of learners, as well as the ways in which these factors intersect with learners' experiences of cultural sustenance within their holistic STEM education ecosystems. I base the significance of this problem on the racial and gender inequities evident in the STEM fields and the social and cultural dynamics that discourage members of these groups from pursuing STEM endeavors even when interest, goals, and self-efficacy may exist. To explore the nuances of this fundamental issue, I outline a critical quantitative survey design research study grounded in a multifaceted complexity/critical theoretical framework. Through this lens, I examined my problem of practice with regard to its ramifications for teaching and learning, with findings suggesting relatively consistent levels of self-efficacy and cultural sustenance across the STEM ecosystem. Six strands of science learning impacts, however, varied significantly in interesting ways that call into question the conceptualization most common in the informal STEM learning field. Through this study, my goal was to inform a meaningful, authentic alignment with the perspectives, needs, motivations, and strengths of learners, supporting equitable, responsive, holistic access to STEM learning opportunities and a disruption of the persistent trends of underrepresentation in these fields.
\end{abstract}




\section{Dedication}

This manuscript and the research it reflects are dedicated to my children, Aria and Westley, with eternal gratitude for the joy and laughter you bring to my life. While it may yet be many years before you read this, I hope you always know and never doubt just how much I love you. 
Acknowledgements

I gratefully acknowledge the thoughtful and patient guidance and support of my dissertation committee, Drs. Anita Bright, Micki Caskey, Amanda Sugimoto, and Alma Trinidad. You are and will always be inspirations to me, and I look forward to continued collaborations in the years ahead.

To Anita Bright, who (wisely or not...) agreed to serve as my advisor and committee chair, I cannot express how thankful I am both for your kind encouragement throughout the craziness of the past years and your gentle but persistent pushing to ensure continued progress. You are amazing, and a guiding light to me and so many others!

To Alma Trinidad, I count myself as beyond fortunate to have had the incredible honor of working with and learning from you during not only my doctoral studies, but during my earlier master's research. Thank you for walking with me on my academic journey for nearly ten years - it has been a true pleasure and privilege.

To the instructors and students who supported this research through your participation, I am forever in your debt. This study was only made possible thanks to your willingness to contribute your time and wisdom to someone you had never met, and I am humbled by your graciousness in choosing to do so.

To my OMSI colleagues past and present, I will never stop believing on some level that there was some great cosmic mix-up that has granted me the opportunity to get paid to work with a team that helps me to learn new things literally every day, and to continue to grow and challenge my assumptions. I have no idea how I got so lucky. 
To Ana Lapite-Cardiel, thank you for your support throughout this wild journey, and for being such a wonderful mother to our sweet little girl. I am deeply fortunate to have you as part of my team.

To my family, including my mom, my dad, my aunt Wendy, my uncle Len, my cousin KJ, and the many others who have for the past four and a half years put up with dissertation-related disruptions with patience, grace, and love, thank you. Without you, I would never have been able to make it through; now, let's celebrate!

To my sibling Em, in particular, you know how much your presence in my life has meant and continues to mean to me, and I hope you recognize how much you inspire me. We share a lifetime of ridiculous memories, and I look forward to more down the road, particularly now that you live here in the Northwest again.

And to Brea Justine Cardiel (née Cohen), words could never express what your partnership, your support, your love, and your unshakeable faith in me have meant. You have gently pushed me and helped me to learn and grow in countless ways, and it fills my heart to overflowing to walk through life with you. I love you, always forever. 
Table of Contents

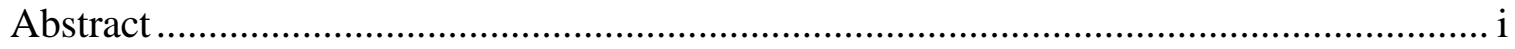

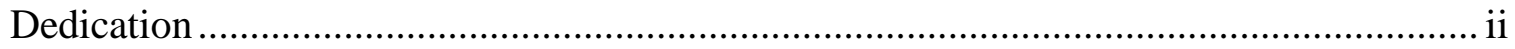

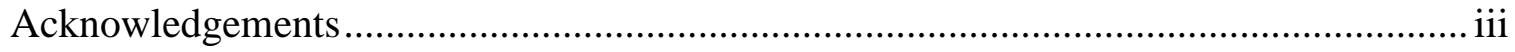



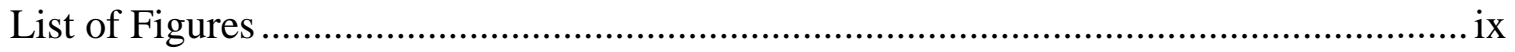

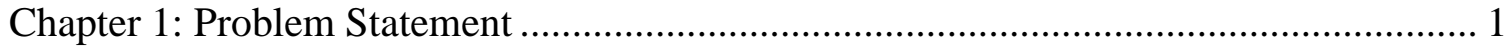

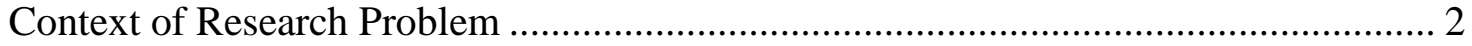

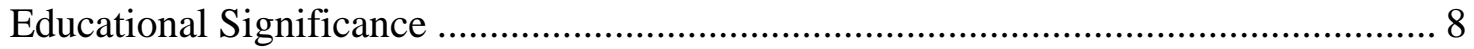

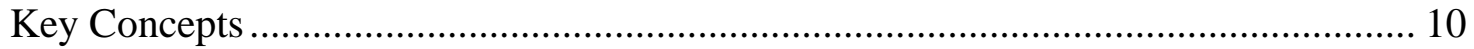

Communities underrepresented in STEM.......................................................... 11

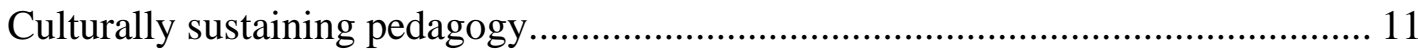

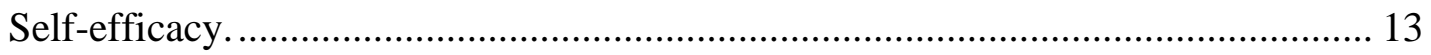

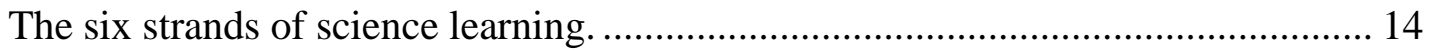



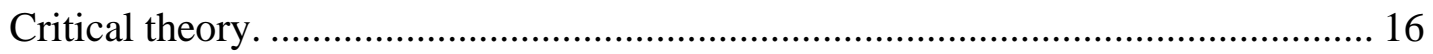

Research Questions and Overview of Methods .................................................. 17

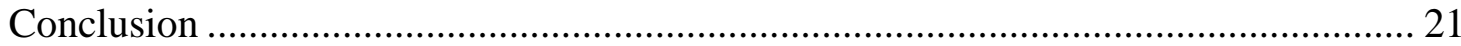



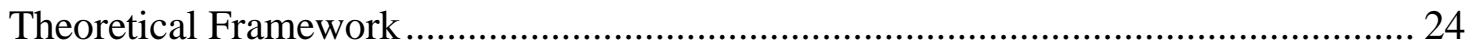

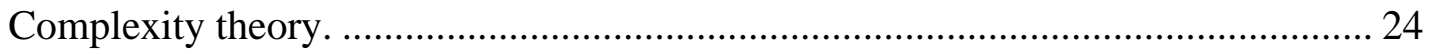

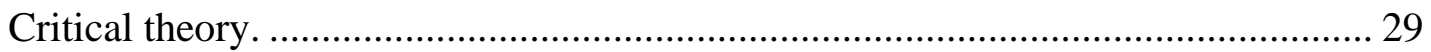

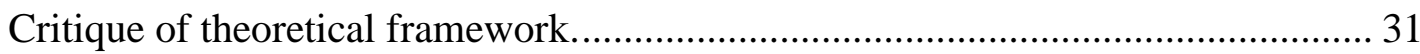

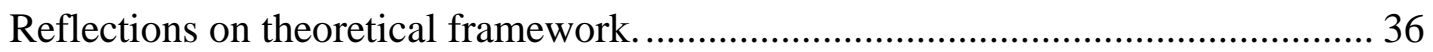

Review of Research Literature..................................................................... 41

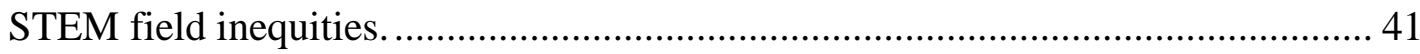

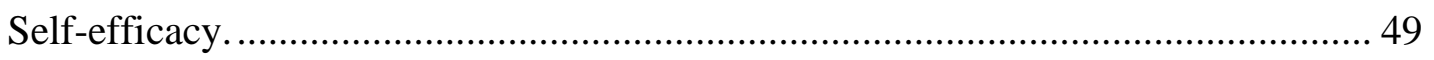



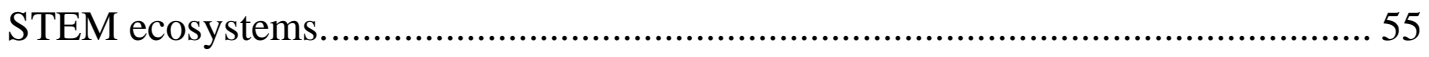



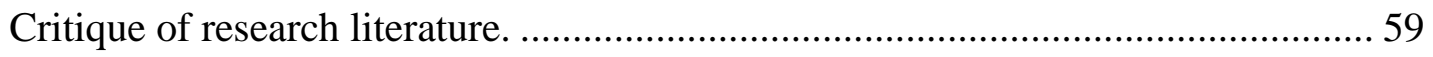

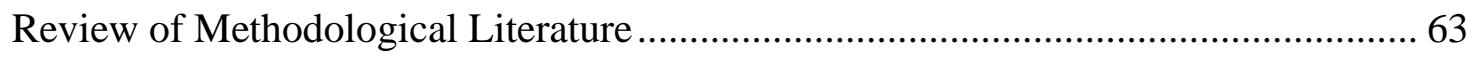




A MULTIPLICITY OF JOURNEYS vi

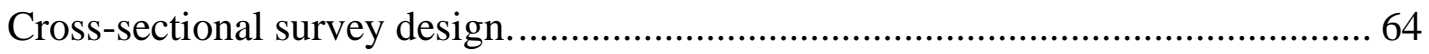



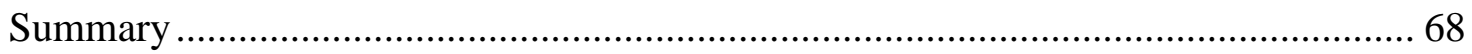

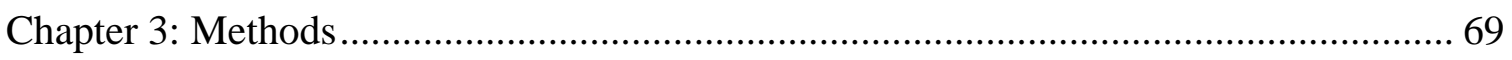

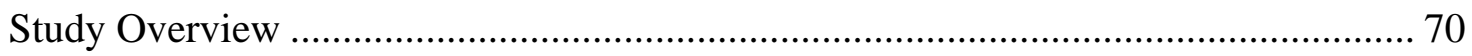

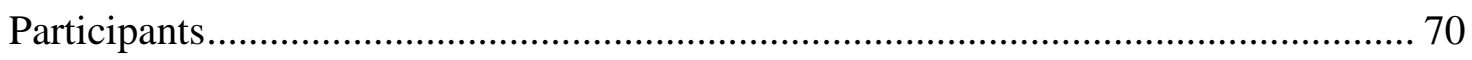

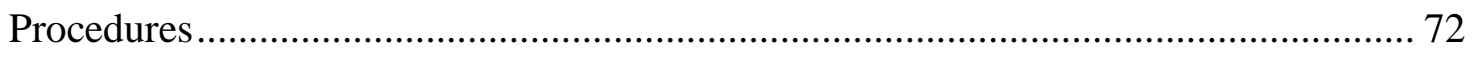

Instruments and Measures.............................................................................. 74

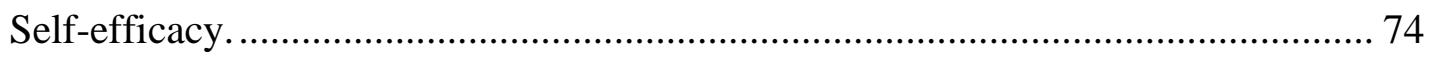



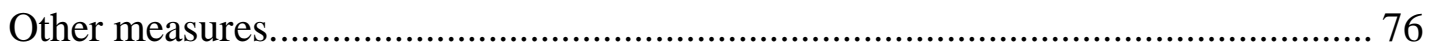

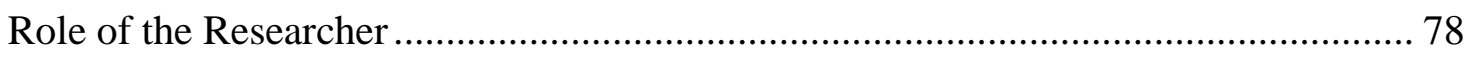

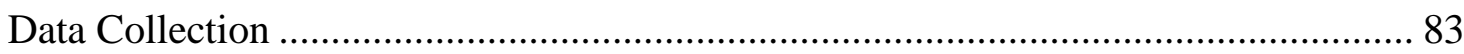

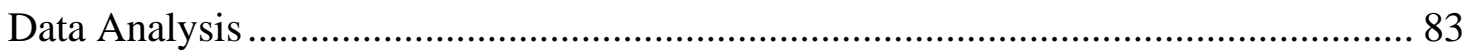

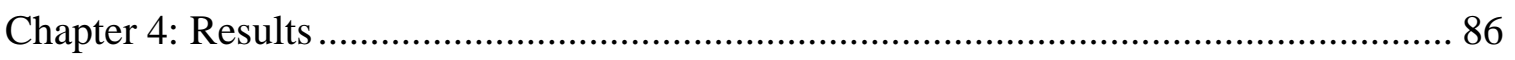



Presentation and Analysis of Survey Data............................................................. 88

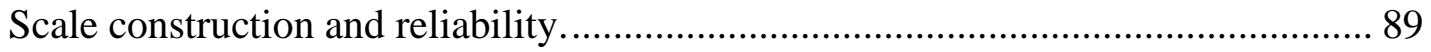

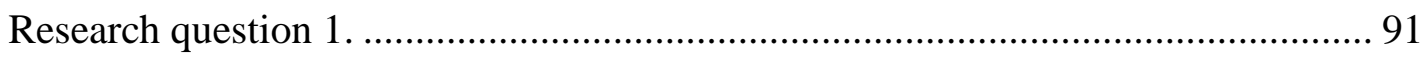

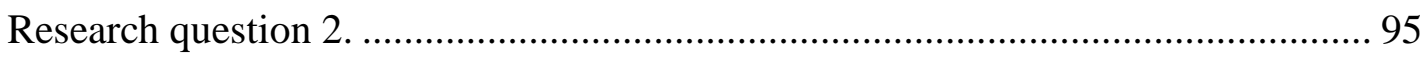



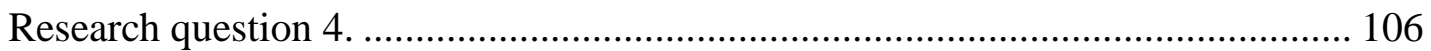

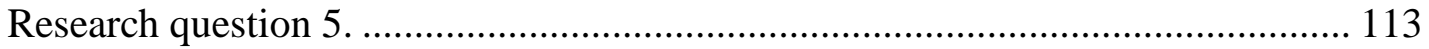

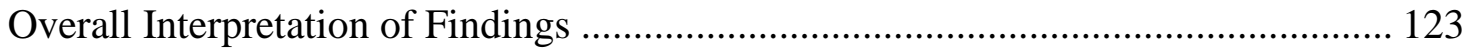

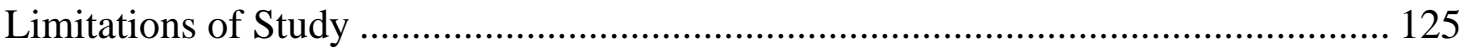

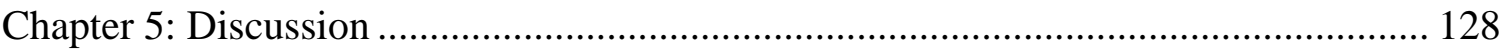

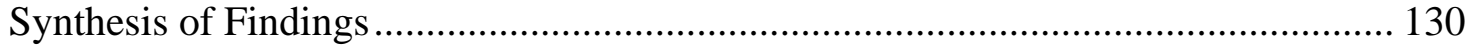

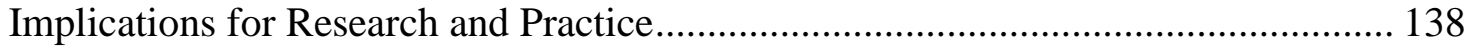

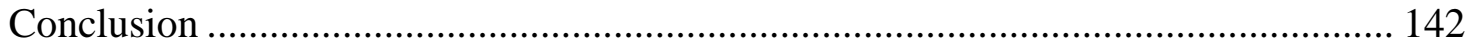

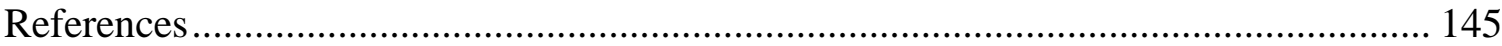

Appendix A: Parent/Guardian Informed Consent Letter .......................................... 176

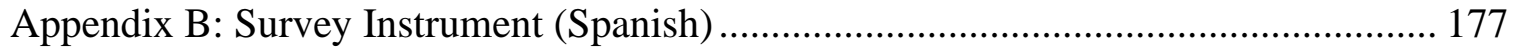

Appendix C: Survey Instrument (English) .......................................................... 183 
A MULTIPLICITY OF JOURNEYS vii

Appendix D: Established Self-Efficacy Measures .................................................... 189

Appendix E: Established Culturally Responsive Teaching Measures .......................... 192

Appendix F: Culturally Sustaining STEM Learning Experiences Scale ...................... 200 


\section{List of Tables}

Table 1: Six Strands of Science Learning Descriptions................................................. 14

Table 2: Statistical Analyses, By Research Question .............................................. 85

Table 3: Initial Scale Composition and Reliability Assessments, Cronbach's $\alpha \ldots \ldots \ldots \ldots . . .89$

Table 4: Final Scale Composition and Reliability Assessments, Cronbach's $\alpha$............. 91

Table 5: Self-Efficacy Descriptive Information by Ecosystem Resource Type .............. 93

Table 6: Self-Efficacy Descriptive Information by Gender and Ecosystem Resource Type

Table 7: Self-Efficacy Descriptive Information by Race and Ecosystem Resource Type 99

Table 8: Self-Efficacy Descriptive Information by Ethnicity and Ecosystem Resource

Type

Table 9: Self-Efficacy Descriptive Information by Race (Binary) and Ecosystem

Resource Type 101

Table 10: Culturally Sustaining Learning Experience Descriptive Information by

Ecosystem Resource Type 104

Table 11: Culturally Sustaining Learning Experience Descriptive Information by Gender and Ecosystem Resource Type

Table 12: Culturally Sustaining Learning Experience Descriptive Information by Race and Ecosystem Resource Type Error! Bookmark not defined.

Table 13: Culturally Sustaining Learning Experience Descriptive Information by Ethnicity and Ecosystem Resource Type....

Table 14: Culturally Sustaining Learning Experience Descriptive Information by Race (Binary) and Ecosystem Resource Type.

Table 15: Six Strands of Science Learning Scale Information by Ecosystem Resource Type

Table 16: Six Strands of Science Learning Survey Item Information by Ecosystem 


\section{List of Figures}

Figure 1: Visual representation of percentage of lifelong STEM learning occurring in formal classroom settings. Originally presented in Falk and Dierking, 2010

Figure 2: STEM Ecosystems learner-centered framework of educational resources and stakeholders. Originally presented in STEM Funders Network, 2016.

Figure 3: Conceptual model of proposed study, including location of research questions in relationship to key concepts and variables.

Figure 4: Overall distribution of self-efficacy scale scores across ecosystem resource type

Figure 5: Overall distribution of culturally sustaining learning experience scale scores across ecosystem resource type

Figure 6: Overall distribution of six strands of science learning scale scores across ecosystem resource type

Figure 7: Q-Q Plots of Symmetry of Six Strands Scale Data Distribution, Differences Between Ecosystem Resource Area. 


\section{Chapter 1: Problem Statement}

As I was growing up, my family’s visits to informal education institutions, first

the Pacific Science Center and Seattle Children's Museum and then the Oregon Museum of Science and Industry (OMSI), were integrally important in sparking my curiosity regarding science and the natural world and instilling what has become a lifelong passion for learning. Valuable though such experiences were to me, I recognized to some degree that my friends and acquaintances did not universally share them. However, it was not until much later, when I entered the field of informal STEM education (ISE) as a professional, that I became more deeply aware of the ways in which these experiences were influenced by my identity as a white male from a middle-class background. From male high school students expressing greater interest in engineering and technology than female students (Cook, Mason, Morse, \& Neuhauser, 2015) to persistent gaps in degree attainment between learners of difference races (National Science Foundation, National Center for Science and Engineering Statistics [NSF NCSES], 2017) to significant disparities between women and men in the workforce (National Science Board [NSB], 2016), the trends of inequitable representation in education and industry on the basis of gender, race, and (dis)ability within the fields of science, technology, engineering, and math (STEM) are substantial and widespread. While incremental improvements have been observed in some cases - for example, the share of science and engineering jobs held by women increased from $29 \%$ to $39 \%$ from 2013 to 2016 (NSB, 2016) - the overall picture remains far from equitable. Clearly, a fundamental problem exists: Learners are not engaging in STEM or being supported in fostering STEM interest at equitable rates, and we as STEM educators do not sufficiently understand the individual and cultural 
motivations, needs, and agentic behavior of learners, nor how learners engage with resources and providers within their holistic STEM ecosystems to avail themselves of culturally sustaining experiences and resources as they chart their own educational journeys.

With all this in mind, I am deeply cognizant of the fact that I have been offered opportunities that are not guaranteed to others who are equally deserving, and while I cannot shed the unearned layers of privilege I possess in United States society, I can attempt to use these privileges to illuminate, question, and perhaps destabilize the underlying systems of inequity. Building on this personal imperative, the purpose of my dissertation study was to explore the dynamics and cultural underpinnings of STEM learner attitudes, as well as how these attitudes and cultural backgrounds are sustained by various opportunities within the holistic Portland-area STEM education ecosystem and map to a range of STEM learning outcomes. In the following pages, I provide evidence of this problem and outline the implications thereof for both formal and informal educators, and also begin demarcating the theoretical and practical boundaries within which I positioned my exploration.

\section{Context of Research Problem}

I approached the study of this research problem with an understanding that learning takes a multitude of forms and occurs within and across an immensely complex and varied landscape of educational contexts. With this in mind, in addition to applying a theoretical framework that directs me to remain attuned both to the complexity of STEM learning and to the humanity, agency, and identities of the people who experience and pursue it, I felt it was vital that I take a systemic approach to conceptualizing learning 
space. In recent years, the STEM Funders Network (2016) developed a framework of STEM education ecosystems to guide community organization efforts around young people's STEM learning. Within this framework, STEM education ecosystems comprise a range of formal and informal learning resources, including industry, family, and out-ofschool providers. The STEM education ecosystem framework has been primarily implemented only within the context of the STEM Funders Network; however, its applicability to my problem of practice is clear. The application of an ecosystems framework is of fundamental importance in exploring the decisions made by learners as active agents in their personal educational journeys - yet, I intended to remain attentive to the potential insufficiency of the model proposed by the STEM Funders Network. The process by which categories of STEM education providers and resources were identified is unclear, and I fully acknowledged that these categories may either be incomplete or fail to resonate with the perspectives, experiences, and goals of learners themselves.

Informal STEM education exists at the nexus of the broader fields of STEM education, including formal instruction as well as informal education, i.e., learning outside school contexts, encompassing the approximately $85 \%$ of learning that takes place beyond the classroom and similar formal education settings (Cross, 2006). Indeed, Falk and Dierking (2010) posited that in STEM education in particular, as much as 95\% of lifelong learning occurs in out-of-school spaces (see Figure 1). 


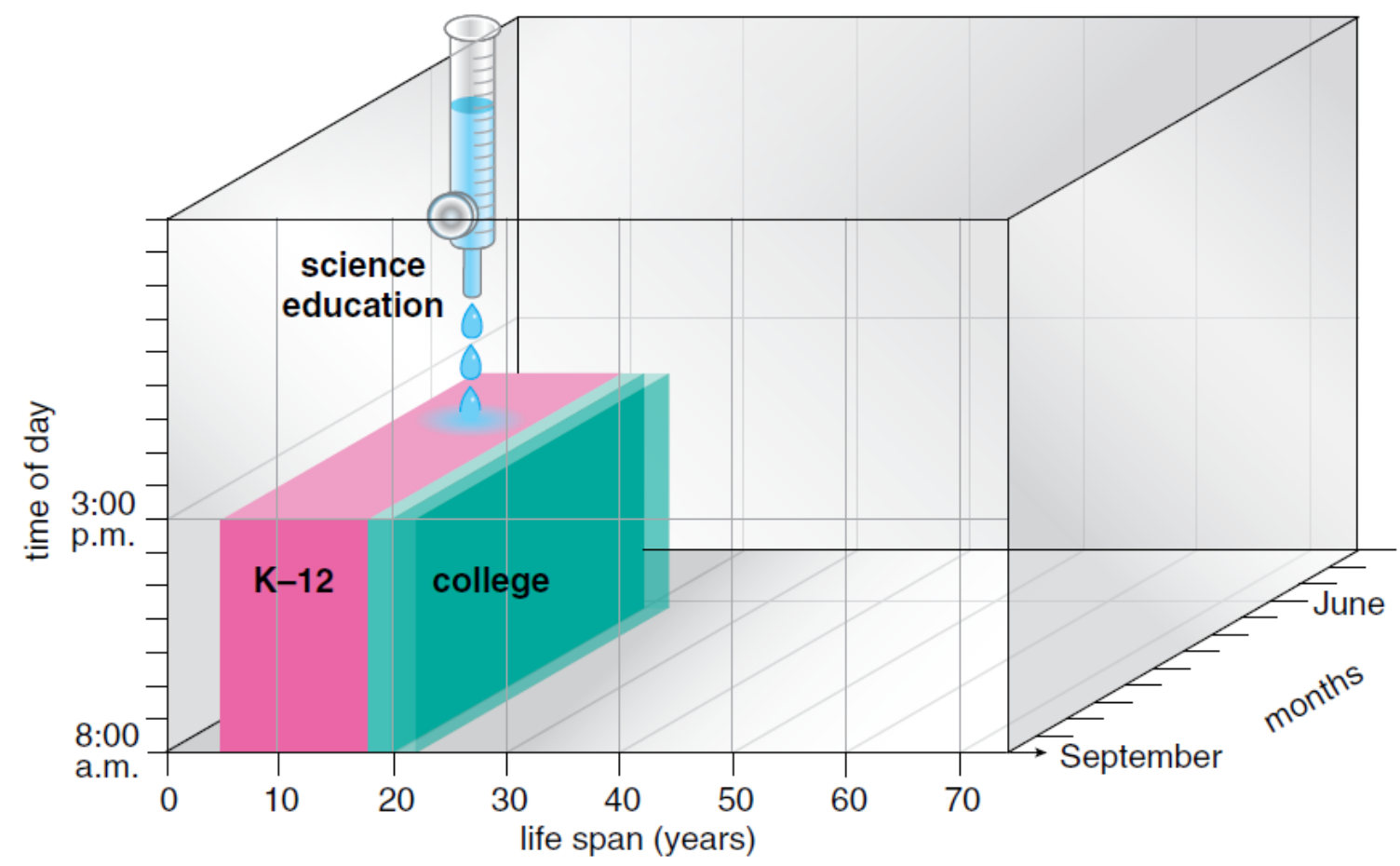

Figure 1. Visual representation of percentage of lifelong STEM learning occurring in formal classroom settings. Originally presented in Falk and Dierking, 2010

Informal STEM education, or ISE, can be used to refer to "science museums, community-based organizations with mandates for informal STEM learning, STEMoriented television, film, books and after-school programs and the Internet" (Falk, Randol, \& Dierking, 2012, p. 866), among other settings. In the context of my study, informal STEM education was of particular interest due to (a) the aforementioned weighting of lifelong STEM learning that occurs in non-classroom settings, (b) the relative lack of extant research regarding self-efficacy and culturally sustaining pedagogies in the informal STEM realm, and (c) my positionality as an informal STEM researcher and practitioner which drove me to ensure the findings of this study were relevant and actionable for educators occupying non-traditional roles in the Portland-area 
STEM ecosystem. While the world of informal STEM learning is enormously complex and varied, encompassing a myriad of forms of out-of-school engagement and education (both structured and unstructured), my personal connection is through the work of science centers and museums, particularly that of OMSI, my current professional home.

Science centers, also referred to as science museums, are defined by their staff's mission of connecting individuals with science, providing firsthand experiences related to scientific concepts and phenomena, and encouraging curiosity among those who visit (Association of Science-Technology Centers [ASTC], 2016). Identification as a science center is largely at the discretion of a given institution and its staff, but in most or all cases, the visions and activities of such institutions will align with these characteristics. In articulating its purpose and charge, the primary professional organization dedicated to science centers and similar ISE providers in the United States indicates that it serves "science centers, museums, and related institutions, whose innovative approaches to science learning inspire people of all ages about the wonders and the meaning of science in their lives" (ASTC, 2013, p. 1). This goal aligns with the OMSI mission statement, which is to "inspire curiosity through engaging science learning experiences, foster experimentation, and the exchange of ideas, and stimulate informed action" (OMSI, n.d.), and offered a conceptual foundation for situating and considering my problem of practice.

Although science centers provide education and exposure to STEM-related content and experiences to people who visit (Falk \& Dierking, 2010; National Research Council [NRC], 2009), only one in four adults in the United States reported visiting a science center within the past year (NSB, 2014). While data regarding youth are difficult 
to locate if they exist at all, the low rates of adult visitorship place clear constraints on attendance among children; field trips and outreach programming provide additional avenues for youth engagement, but while such systems are valuable, family group visits result in more positive learning outcomes than do individual and non-family group visits, as well as opportunities for adult caregivers to support children's interests and identity formation (Riedinger, 2012). Of even greater concern is that among certain audiencessuch as those with lower levels of formal educational attainment—as few as $8-16 \%$ of the members of these audiences indicated that they have visited a science center within the past year (NSB, 2014, 2016). As previously noted, what many in the ISE field have been grappling with is the fact that in the majority of cases, the learners who visit our institutions are unrepresentative of the larger communities in which these institutions are located. I find this dynamic particularly troubling, as I believe it may speak to a misalignment of science center offerings with the goals and motivations of those we hope to serve. Based on OMSI’s 2013 study of demographics during "\$2 Days" (the first Sunday of each month, during which general admission costs are lowered to \$2.00), at our museum specifically, our visitors are disproportionately wealthy (i.e., reporting a median household income above $\$ 60,000 ; 57.3 \%, n=75$ ) and are primarily white $(78.5 \%, n=106)$ and non-Hispanic (95\%, $n=133)$ (Walther, Cardiel, \& Reyes, 2014). Again, I find these trends worrisome, not only from the standpoint that true equity of access is a meaningful goal, but more specifically due to the findings of past research (Falk \& Dierking, 2010; Falk et al., 2016; NRC, 2009) indicating that science centers offer opportunities for individuals of all ages to learn about STEM and develop identities as STEM learners to the degree that they desire to do so. This is a meaningful 
distinction, as STEM learning takes place in a variety of contexts and is not constrained to formal school settings.

Given that the STEM fields, while historically and in many cases contemporarily problematic, also hold potential for empowerment, liberation, and cultural sustenance, this inequitable representation of audience groups among science center visitors is itself a phenomenon deserving of exploration; however, of equal concern are the ways in which professionals within the field of informal STEM education (ISE) have approached its redress. Many individuals from a multitude of ISE organizations have dedicated substantial effort to initiatives focused on increasing visitorship and participation by traditionally underrepresented audiences, and research has been and continues to be conducted in support of such initiatives. However, what appears to be largely lacking from the current research is a motivations-focused (Ford, Brickhouse, Lottero-Perdue, \& Kittleson, 2006; Maltese \& Tai, 2010; Vedder-Weiss \& Fortus, 2011) exploration of how learners understand, navigate, and avail themselves of opportunities and resources within their holistic STEM education ecosystems described below (STEM Funders Network, 2016; see Figure 2). I intended to focus my efforts on the STEM ecosystem inhabited and navigated by Portland-area learners. This STEM ecosystem includes informal organizations such as OMSI and the Portland Children's Museum, as well as schools, out-of-school program providers, households, and STEM businesses. 


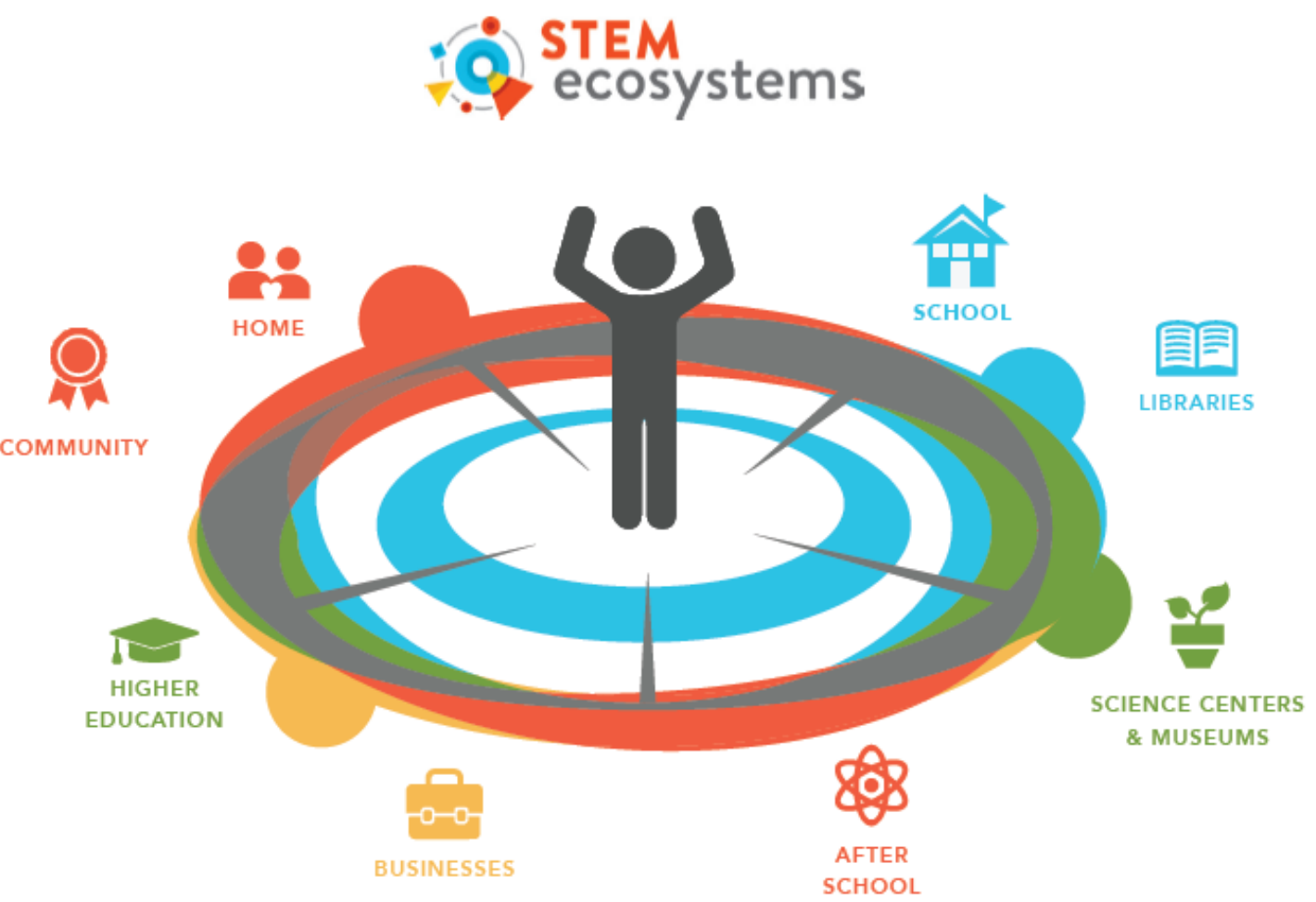

Figure 2. STEM Ecosystems learner-centered framework of educational resources and stakeholders. Originally presented in STEM Funders Network, 2016.

\section{Educational Significance}

Highlighting the persistent inequities in STEM education and career tracks is not to imply that every individual should focus only on STEM topics to the exclusion of other pursuits; learners should feel empowered to explore and refine their interests in ways that are true to their unique identities. Creating culturally sustaining STEM ecosystems requires educators to recognize and support the agency of learners and the legitimacy of their pedagogical preferences, as well as the value of other resources within their ecosystem. It is for this reason that the persistent disparities in STEM representation are fundamentally problematic — rather than arising from essential 
differences in interests between groups, there is evidence that they result from the complex and problematic interplay of multiple systems of privilege and power that limit the support, encouragement, and empowerment certain learners receive in their educational journeys. Fouad and Santana (2017) noted:

Women and racial-ethnic minorities are not choosing to enter-or stay in-STEM careers at the same rate as men and racial majority persons. Their lower rates of entrance into STEM fields may not be related to a lack of interest or intention...Hanson [2004] suggests that both racism and sexism may act as deterrents from science involvement for African American women. (p. 27) In other words, it is vital that we understand that interest and self-efficacy, while related, are separate and distinct variables, and that the lived experiences of female learners and learners of color may impact each variable in different and meaningful ways. If, as Gillborn (2016) posited, the United States formal education system functions implicitly (and occasionally explicitly) to perpetuate hegemonic power dynamics, it follows that STEM learners within these systems may have very different experiences-including degrees of support for their motivations and self-efficacy regarding STEM topics-on the basis of race and gender.

LeGrand (2013) noted that although interest in STEM topics among K-12 students was sometimes inconsistent between genders, these inconsistencies were not always in favor of male students, with female students expressing greater interest in several topics and at several grade levels. By contrast, boys expressed higher levels of both self-efficacy and performance expectancy across nearly all grade levels and topics. Mediating the relationship between gender and STEM interest and involvement is 
perceived alignment with one's values and motivations; researchers have argued that women and girls are more likely to orient toward occupations, including those in the STEM fields, that focus on helping others than to those that emphasize agentic achievement (Bandura, Barbaranelli, Caprara, \& Pastorelli, 2001; Barth, Guadagno, Rice, Eno, \& Minney, 2015; Ceci \& Williams, 2011; Jones, Howe, \& Rua, 2000; Thom, 2001). Conversely, Hardin and Longhurst (2016) emphasized the reality and significance of stereotype threat (Steele \& Aronson, 1995), a phenomenon wherein "the anxiety arising from the fear of confirming a negative stereotype about one's group interferes with performance" (Hardin \& Longhurst, 2016, p. 235). Taken together, studies such as these illustrate what I held to be a key distinction: Interest and a desire to learn about and engage with STEM topics may be present to roughly equal degrees across demographic groups (acknowledging the existence and legitimacy of variations in individual preference). However, the experiences of female learners and learners of color in the United States have the potential to differ substantially from male and White learners, and these differences in lived experience may affect the degree and types of support they receive for their STEM learning motivation and self-efficacy. Furthermore, the resultant differences in individual experiences, attitudes, and goals may contribute to differing educational outcomes as articulated in the six strands of science learning (NRC, 2009) across the Portland-area STEM education ecosystem.

\section{Key Concepts}

To best explore the underlying causes and themes and to demarcate my specific theoretical and conceptual framework, I situated my study within the extant body of relevant literature. In this section, I provide an overview of key concepts germane to the 
problem and its study, including those related to my variables of interest. In Chapter 2, I further explicate the key concepts individually and in relation to one another and the research questions posed below.

\section{Communities underrepresented in STEM. Communities underrepresented in}

STEM, for the purposes of this study, refers to those groups of learners and professionals who, based on current and recent surveys of STEM education (including K-12 and all levels of higher education) and career tracks, are present at disproportionately low levels. As defined by the National Science Foundation (NSF, 2015), "women, persons with disabilities, and three racial and ethnic groups_-blacks, Hispanics, and American Indians or Alaska Natives - are underrepresented in [science and engineering]" (p. 2). While arguments could be made for the inclusion of other groups in this definition (notably, learners from rural communities and those from households below the poverty line), this proposal adheres to the delimitations outlined by the NSF. Bearing in mind the cautions voiced by contemporary critical researchers (e.g., Basile \& Lopez, 2015; Mansfield, Welton, \& Grogan, 2014) regarding the framing of underrepresented communities in policy documents, my intention in using the NSF's definitions was only to delineate the boundaries of inquiry for this study rather than to suggest that learners from these communities should by definition be directed to the STEM fields regardless of personal interest, self-efficacy, or educational goals.

Culturally sustaining pedagogy. Proposed in 2012 by Django Paris as a framework for educational engagement with learners from minoritized cultures, culturally sustaining pedagogy builds upon the concepts of culturally responsive pedagogy (Ladson-Billings, 1995), funds of knowledge (Moll, Amanti, Neff, \& 
Gonzalez, 1992), and other "resource pedagogies" (Paris \& Ball, 2009) through the integration of cultural pluralism. This framework acknowledges the importance and value of culturally responsive pedagogical approaches but holding the position that such approaches fail to sufficiently support and sustain learners' cultural backgrounds and perspectives while concurrently equipping them with the knowledge and tools to navigate the dominant culture successfully. To achieve this goal, culturally sustaining pedagogy "seeks to perpetuate and foster - to sustain—linguistic, literate, and cultural pluralism as part of schooling for positive social transformation” (Alim \& Paris, 2017, p. 1). Practitioners of culturally sustaining pedagogy recognize and foreground the agency of learners from diverse cultural backgrounds and the legitimacy of the knowledge and worldviews they bring to the classrooms, museums, and other learning spaces through which their journeys take them. Interestingly, ecosystemic perspectives on learning and culturally sustaining pedagogical approaches hold a shared potential to destabilize hegemonic systems of power within educational systems, a fact that has not gone unnoticed by scholars and practitioners. As noted by Lee (2017), "culturally sustaining pedagogy [is] rooted in an ecological frame, in the sense that [it is] asking teachers to take into account aspects of youths' lives outside the classroom not only as resources, but as targets of learning to be sustained" (p. 262). I was delighted that contemporary researchers have identified this point of congruence, as it mirrored my own belief that an ecosystemic approach to education is almost by definition a framework for cultural sustenance of learners, and was eager to contribute to the advancement of theoretical and practical knowledge related thereto through my dissertation study. 
Self-efficacy. Closely related to the concept of achievement goals and educational motivation (AL-Baddareen, Ghaith, \& Akour, 2015; Bjørnebekk, Diseth, \& Ulriksen, 2013; Bong, 2001; Jiang, Song, Lee, \& Bong, 2014) and emerging from Bandura's (1971, 1977, 1986a) theory of social cognition, self-efficacy refers to an individual's confidence in their ability to be successful in a given undertaking. Selfefficacy has seen wide use in research conducted across a range of social science disciplines; in the field of education, it has been assessed in relation to career aspirations (Bandura et al., 2001; Tang, Pan, \& Newmeyer, 2008), STEM interest and outcomes of pursuing a STEM degree (Fouad \& Santana, 2017; Hardin \& Longhurst, 2016), and academic achievement more broadly (Affuso, Bacchini, \& Miranda, 2017; Bjørnebekk et al., 2013; Caprara, Vecchione, Alessandri, Gerbino, \& Barbaranelli, 2011; Merolla, 2017; Reid, 2013), among other concepts. In this study, I positioned self-efficacy as a potentially significant driver in motivating learners to avail themselves of certain STEM education resources and opportunities while selectively declining to participate in others. It would be inaccurate to claim that self-efficacy alone provides a comprehensive framework for understanding agentic navigation of STEM ecosystems - for example, as noted by Bandura, Barbaranelli, Caprara, and Pastorelli (1996), the relationship between self-efficacy and academic functioning is complex and is mediated by, at a minimum, parental and child academic aspirations, learner social efficacy and degree of prosocial behavior, peer preference, and socioeconomic status. Furthermore, I would suggest we could safely add characteristics such as race, gender, and ethnicity to this list of variables. Nonetheless, while the inclusion of self-efficacy was not sufficient, it was necessary for a complete picture of STEM learning to be painted. 
The six strands of science learning. Providing another framework that is useful for examining my problem of practice, the six strands of science learning (NRC, 2009) outlined in Table 1 articulate a succinct list of science-specific outcomes that may be supported by ISE professionals.

Table 1

Six Strands of Science Learning Descriptions

\begin{tabular}{clc}
\hline Strand \# & \multicolumn{1}{c}{ Description } & ISE Focus \\
\hline Strand 1 & $\begin{array}{l}\text { Experience excitement, interest, and motivation to learn } \\
\text { about phenomena in the natural and physical world }\end{array}$ & X \\
Strand 2 & $\begin{array}{l}\text { Come to generate, understand, remember, and use } \\
\text { concepts, explanations, arguments, models, and facts } \\
\text { related to science }\end{array}$ \\
Strand 3 & $\begin{array}{l}\text { Manipulate, test, explore, predict, question, observe, and } \\
\text { make sense of the natural and physical world }\end{array}$ & \\
Strand 4 & $\begin{array}{l}\text { Reflect on science as a way of knowing; on processes, } \\
\text { concepts, and institutions of science; and on their own } \\
\text { process of learning about phenomena }\end{array}$ & \\
Strand 5 & $\begin{array}{l}\text { Participate in scientific activities and learning practices } \\
\text { with others, using scientific language and tools }\end{array}$ & \\
Strand 6 & $\begin{array}{l}\text { Think about themselves as science learners and develop } \\
\text { an identity as someone who knows about, uses, and } \\
\text { sometimes contributes to science }\end{array}$ & X \\
&
\end{tabular}

Rather than serving as a high-level theoretical framework per se, the six strands "represent the ideal that all institutions that create and provide informal environments for people to learn science can strive for in their programs and facilities" (NRC, 2009, p. 43) 
and, resultantly, offered a conceptual bridge to facilitate the interpretation of findings to an educational context (informal STEM learning) which is not well-represented to date in the self-efficacy literature.

Complexity theory. Understanding the interconnectivity and interdependence of myriad systems and subsystems and the diverse learners and educators within the Portland-area STEM ecosystem, the framework of complexity theory provided valuable insights into the dynamics underlying agentic movement throughout this ecosystem. In 2003, De Laat and Lally argued that no single theoretical framework had yet emerged which was capable of providing what they considered a robust articulation of description, rhetoric, inference, or application as pertaining to education, and specifically to networked learning environments. De Laat and Lally were conceiving of "networked learning" as referring specifically to educational contexts, particularly those in higher education, that involved an online delivery and engagement mechanism-i.e., "networked" in the sense of computer networks. However, I would posit that the lay definition of "networked" as "connected with multiple other individuals, groups, and systems" is equally valid and appropriate in this context. In considering the possible reasons for what they argued to be a fundamental shortcoming, De Laat and Lally referred repeatedly to what they perceived as the complexity of praxis and suggested that "perhaps we have not yet, as a research community, fully and openly acknowledged the complexity of researching the central educational processes of learning and teaching" ( $\mathrm{p}$. 9). It is to this end-acknowledging and exploring the enormous complexity of processes and connections inherent in educational and learning systems - that the use of complexity theory is most effectively dedicated. As described by Brack, Lassiter, Hill, and Moore 
(2011), "complexity theory focuses on the patterns that emerge from nonlinear dynamic systems that are always changing over time. In a system, factors interrelate in an intricate and seemingly unpredictable manner" (p. 5). Similarly, Horn (2008) explained, "Complexity focuses on emergent behaviours that result from interactions within and among self-organizing and adaptive systems" (p. 132). At its core, complexity theory (as applied to educational systems) emphasizes that behaviors, interactions, and phenomena cannot be fully understood when considered only within their immediate context; rather, it is necessary to acknowledge and assess the influence of other proximal and distal factors that may not be immediately evident. To this end, during the process of data analysis and subsequent discussion of findings, I intended to apply a complexity lens to carefully consider and, to the greatest degree possible, highlight and address systemic influences as potentially affecting the relationship between the primary variables of interest.

Critical theory. Last, by applying the lens of critical theory to my problem of practice, I intended to confront the realities of power, privilege, and access that are woven throughout every learner's lived experience and contribute to motivations, goals, self-efficacy, and the choices each makes regarding their STEM education journey. As articulated by Robinson (1994), "critical theory promises practitioners and researchers in educational administration a morally based vision of socially just administrative arrangements and the means for achieving them" (p. 56). Critical theorists reject the positivist claim that empirical examinations of reality as an objective experience are ultimately desirable (or even possible) as the goal of research. Rather, critical theorists recognize the subjectivity of lived experience, acknowledging and making visible the 
unavoidable biases brought by researchers and dedicating their efforts to illuminating and suggesting methods of redressing systemic and structural inequities. Critical researchers and theorists have previously turned their attention to science education and STEM (Basile \& Lopez, 2015; Dimick, 2016; Harding, 1998; Mansfield et al., 2014; Peralta, Caspary, \& Boothe, 2013; Sayman, 2013), as well as to educational leadership more broadly (Carlile, 2012; Greenwood, 2010; Henze \& Arriaza, 2006; Ryan, 1998). In addition to being centrally concerned with emancipation of those individuals and groups who endure systemic oppression (Duffy \& Scott, 1998), critical theory places a premium upon the reflective engagement, conscious acknowledgement of positionality on the part of researchers and practitioners, concomitant with a rejection of essentialist interpretations of reality. To this point, Goodkind (2013) noted:

Critical theories in their various forms advocate an epistemological shift from a positivist approach that professes to describe an objective reality and claims to be value neutral to an interpretivist approach that focuses on history, context, experience, positionality, and making overt one's prescriptive beliefs about how the world should be. (p. 396)

Applied to my problem of practice, critical theory emphasized the socially constructed and value-laden nature of STEM education expectations and the power dynamics that are likely to contribute to inequitable representation in the STEM fields.

\section{Research Questions and Overview of Methods}

In my critical quantitative study, I addressed five research questions, all of which were assessed through the use of a survey questionnaire employing a combination of established measures and newly created (but theoretically grounded) scales comprised of 
multiple conceptually congruent items. The specific research questions I explored are as follows:

$\mathrm{RQ}_{1}$ : In what ways do the feelings of self-efficacy expressed by Portland-area learners correspond with the various resource types in their local STEM education ecosystem?

$\mathrm{RQ}_{2}$ : In what ways do race, gender, and ethnicity impact the relationship between expressed self-efficacy and STEM ecosystem resource types among Portland-area learners?

$\mathrm{RQ}_{3}$ : To what extent do Portland-area learners report feelings of cultural sustenance when engaging with the various resource types in their local STEM education ecosystem?

$\mathrm{RQ}_{4}$ : In what ways do race, gender, and ethnicity impact the relationship between feelings of cultural sustenance and STEM ecosystem resource types among Portland-area learners?

RQ5: In what ways do Portland-area learners indicate that their engagement in their various STEM ecosystem resources correspond to the learning outcomes proposed by the Six strands of science learning?

In order to provide a "map" of sorts outlining the connections between the ideas I have introduced up to this point, I developed a conceptual model as a visual representation of the research questions and their relationships to the aforementioned key concepts and variables (see Figure 3). 


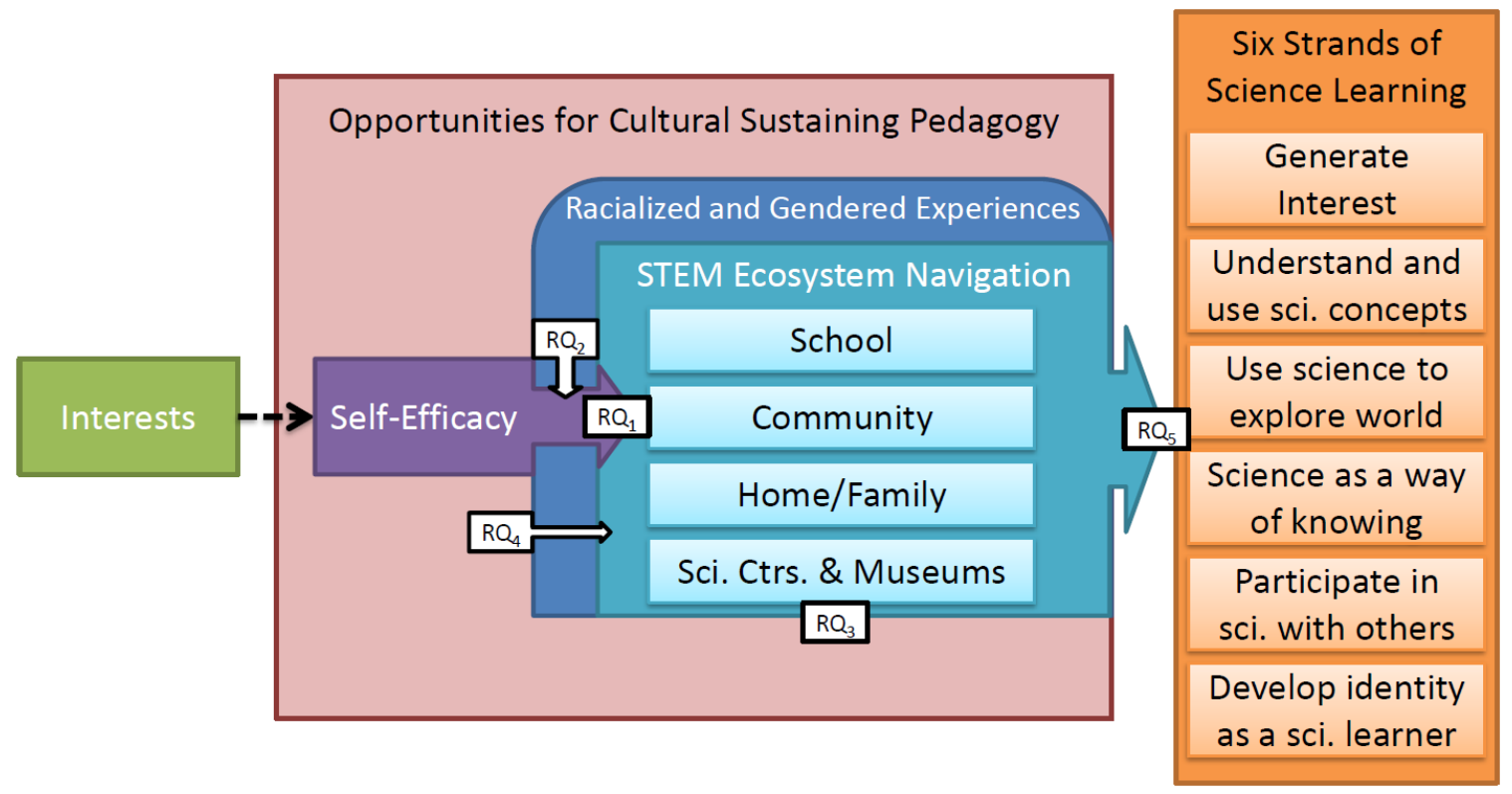

Figure 3. Conceptual model of proposed study, including location of research questions in relationship to key concepts and variables.

Recognizing the complexity of the problem of practice outlined above, the proposed research study incorporated a quantitative methodological approach using a cross-sectional survey design method (Babbie, 1990, 2016; Cornelius \& Harrington, 2014; Creswell, 2014; Fowler, 2014; Nardi, 2018; Visser, Krosnick, \& Lavrakas, 2000) for data collection and descriptive and inferential data analysis. In keeping with the need for a large-scale understanding of perspectives and trends among STEM learners in the Portland area (both those underrepresented and those "proportionately represented" in the STEM fields), the data collection method was a survey questionnaire (Gould, 2011; Savahl et al., 2015) relying upon researcher-administered questionnaires as a method of collecting data from Portland residents. For this survey, I specifically focused upon youth aged 14 to 18 years old recruited in a school district in Oregon's Willamette 
Valley. This age group was selected on the basis of alignment with OMSI visitorship, and is further supported by the body of scholarship that suggests that interest in pursuing a STEM career is strongly influenced by experiences prior to the age of 14 (Jiang, Simpkins, \& Eccles, 2020; Kitchen, Sonnert, \& Sadler, 2018; Sahin, Ekmecki, \& Waxman, 2017; Wang, 2013). Prior research provided robust evidence that children as young as second and third grade are fully capable of possessing and demonstrating agency in their journeys of learning, both in STEM fields and in general (Adair, 2014; Varelas, Kane, \& Wylie, 2012; Varelas, Tucker-Raymond, \& Richards, 2015), offering further support for the selection of this age group as appropriate to the design and intent of the proposed study.

Survey instruments included measures assessing perceptions of cultural sustenance and self-efficacy across multiple domains of the Portland-area STEM learning ecosystem, as well as items corresponding to the outcomes proposed by the six strands of science learning, and a number of basic demographic questions. When possible, measures were drawn from previously-developed and validated instruments (e.g., Bandura, 2006b; Pintrich, Smith, Garcia, \& McKeachie, 1993). However, given the paucity of empirical research employing the frameworks of STEM ecosystems or the six strands of science learning, as well as the absence of quantitative measures designed to assess the relatively recent construct of culturally sustaining pedagogy—particularly as experienced by learners rather than as implemented by educators-I also developed and assessed the reliability of new measures as appropriate. 


\section{Conclusion}

The preceding pages have spoken to the boundaries of a key research problem faced by the informal education field, and indeed by the field of STEM education writ large. Through the complementary theoretical frameworks of complexity theory and critical theory, to serve our communities in an inclusive and culturally sustaining manner, ISE professionals must position our efforts to support and engage with learners within a framework of efficacy and self-directed goals, understanding individuals as active agents in navigating their STEM learning ecosystems, complex and fraught with power dynamics though these ecosystems may be. Furthermore, we can no longer neglect the promise of a culturally sustaining pedagogical approach to STEM education, not only for empowering and (re)centering learners as co-constructors of each educational exchange, but in fact for calling into question the very systems that privilege certain ways of knowing as "STEM" and others as "not-STEM." This reconceptualization of education necessitates not only the mapping of motivations and goals within a varied landscape of opportunities for engagement with STEM content and experiences, but also the positioning of learners as active agents and the ultimate authorities in their own selection of some resources over others.

Building upon this foundation and in recognition of a critical need to respect and respond to the agentic dynamics and decisions of STEM learners, it was vital to focus on developing a nuanced understanding of the goals and attitudes brought by learners that inform their decision to engage (or not) with STEM in various ways within their holistic ecosystems, as well as the form STEM learning takes on the basis of such attitudes, goals, and choices. Given my background, I envisioned the redress of this research problem as 
being of primary value to educators in informal settings, but it may also hold significant ramifications for those in formal settings as well, and most importantly, it may legitimize the motivations and agency of learners as expressed through their STEM education choices. I situated myself fully within the identification and exploration of this problem of practice, recognizing the ways in which my unique background and experiences (as well as my sociocultural markers) have granted me unearned privileges and shaped the beliefs and perspectives I hold. I intended to remain attuned to these dynamics to avoid the reification of the hegemonic power dynamics from which I believed this problem of practice originated. Ultimately, the exploration and discussion of this problem was intended to inform a stronger, more authentic alignment with the perspectives, needs, motivations, and strengths of learners, to ensure equitable, responsive, holistic access to STEM learning opportunities - including STEM experiences imagined, created, and facilitated both by and for diverse communities — and a disruption of the trends of underrepresentation in these fields that have persisted for far too long. 


\section{Chapter 2: Review of Literature}

In preparing to undertake my study, it was necessary to first conduct a thoughtful and thorough review of the extant literature that both bounds and undergirds the problem I wished to address. To reiterate, this problem centered on my belief that learners are not engaging in STEM or being supported in fostering STEM interest at equitable rates, and we as STEM educators do not sufficiently understand the motivations, needs, and agentic behavior of learners to provide culturally sustaining experiences and resources as they chart their own educational journeys. Particularly in light of my ecosystemic approach to exploring the nature and characteristics of learner behaviors and attitudes across educational settings throughout the Portland area, my study required careful attention to the assessment of specific attitudinal and behavioral concepts, recognizing that other concepts lay beyond the scope of this study. Similarly, I focused my study through a specific theoretical framework, which required critical with an eye both to the illumination it affords of certain elements of my research landscape and to its remaining constraints and limitations.

To this point, I begin the following sections of this proposal with a review of the literature regarding complexity theory and critical theory, particularly emphasizing application of these theories to the field of educational research while not disregarding key considerations and foundational learning gleaned from scholarship in proximal disciplines. Next, I explore the literature regarding a well-established construct germane to my study (self-efficacy), as well as pedagogical and interpretive frameworks that are highly relevant but only lightly represented in academic publications to date (STEM ecosystems, culturally sustaining pedagogy, and the six strands of science learning). I 
also drew from the methodological literature to articulate the tenets and expectations of cross-sectional survey design research; my methodology is discussed in detail in the following chapter, but I use this section to offer a research-based justification of its applicability while also identifying its limitations in the present context. In sum, in this chapter I will ground my research problem in the extant body of relevant literature, demonstrate the need for further exploration — to wit, my doctoral study — and lay the foundation for my intended methodological approach.

\section{Theoretical Framework}

Considering the nature of STEM learning in United States society, it is clear that the generation of understanding regarding the nuanced interconnections and broad systemic factors requires an equally nuanced theoretical lens. At the same time, it is vital that educational researchers and practitioners alike acknowledge and explore the sociocultural dynamics of inequity and power inherent to both the process of learning and the fields of STEM. To meet these distinct but equally important requirements, I employed complexity theory in conjunction with critical theory as a combined theoretical framework for planning and conducting my proposed study. These two theories complement one another with remarkable efficacy, and provide a powerful lens with which to examine the motivations and efficacy of learners as active agents within a complex (eco)system of STEM education while concurrently identifying and interrogating the ways in which relevant factors reify or destabilize hegemonic systems of power, privilege, and access.

Complexity theory. STEM learning does not occur in a vacuum; institutions and systems are nested within, and both influence and are influenced by, other institutions 
and systems. Numerous scholars (e.g., Jörg, Davis, \& Nickmans, 2007; Mason, 2008, 2009; Wood \& Butt, 2014) have noted the suitability of complexity theory to studies of education in real-life settings. As Weis et al. (2015) concluded in their study of factors contributing to the ultimate failure of STEM-focused school programs designed to be inclusive of underrepresented student groups, one of the key contributors to the failure of such programs is a lack of recognition of, and unresponsiveness to, external system-level dynamics that influence the efficacy of schools and teachers to enact initiatives as intended. By contrast, complexity can be represented as a radically holistic analysis that does not separate person from context, but shows how all things (individuals, tools, technologies, ideas and environments) are continually brought forth in dynamic systems or 'assemblages' of 'vital materiality' (Bennett, 2009). These systems emerge in unpredictable ways through non-linear dynamics of mutual interaction and influence, producing a whole that is greater than the sum of its parts. (Fenwick, 2012, p. 142)

Furthermore, others have explored the ways in which complexity theory, in combination with ecosystems theory (Bronfenbrenner, 1979), applies to the education of counselors and other conflict resolution professionals (Brack et al., 2011). While these pairings of complexity theory and ecosystems theory have largely occurred outside the field of educational research, they nonetheless offer evidence of what intuitively appears to be a degree of alignment between these theoretical frameworks.

Fenwick (2012) also specified several key principles of complex systems to which educational researchers should attend, including (a) emergence of phenomena, events, 
and actors, which enables self-organization; (b) the presence of nested systems; (c) uncertainty of causes and effects related to non-linear dynamics; (d) internal diversity of agents and subsystems; (e) the presence of positive feedback loops that amplify perturbations; and (f) the ongoing tension between ordering and disordering patterns. Attentive as it is to the range and interrelatedness of systemic factors, the application of complexity theory is also a natural fit for an examination of the myriad players that interact with one another in the realm of politics and educational policy. As alluded to by Mason (2016), understanding the outcomes of a specific educational policy is all but impossible without at least acknowledging and, to whatever extent feasible, disentangling the multitude of systems factors influencing such outcomes, so it is perhaps unsurprising that other researchers and theorists have recognized the potential applicability of this framework for interpreting the many interrelated actors and agencies that operate within the political sphere.

In their multi-site longitudinal research study assessing the dynamics of STEMfocused formal education institutions (in this case, urban high schools in Buffalo, NY, and Chicago, IL), Weis et al. (2015) focused specifically upon programmatic opportunities designed to increase the participation of racial and ethnic minorities in STEM. The researchers predicated their study on the position that many such programs, although doubtless well-intentioned, often fail to effect lasting change. To explore the range of opportunities available, as well as the ways in which these opportunities (a) are perceived by high school professionals and (b) serve to position interested students to further pursue STEM education and careers, Weis et al. conducted artifact analysis of school documents in conjunction with student, teacher, and counselor interviews and 
classroom observations. Analysis of this varied longitudinal dataset yielded a number of findings, including apparent confusion among students resulting from discrepancies between their perceptions of program offerings and the realities of these programs, as well as gradual (or in some cases rapid) degradation of program structure that had substantial negative ramifications for learners. Based on these and several other findings of their analysis, the authors concluded that "enthusiasm and intention to reform STEM education by establishing STEM-focused schools" (p. 1052) did not, in fact, yield the intended outcome in either Buffalo or Chicago. While the findings from this study are not largely positive, Weis et al. noted that schools can best support equitable access to STEM learning opportunities by adopting a systematic perspective-i.e., by planning for, recognizing, and responding to systems-level factors beyond those directly related to programmatic choices (such as student and teacher morale, staffing and resource dynamics, and competing school and district priorities).

The findings of Weis and colleagues' (2015) study are intriguing in their own right, but are particularly troubling when considered in conjunction with the "We've Done Enough" theory of school desegregation (Tushnet, 2016). Essentially, Tushnet (2016) posited that each incremental advance toward racial equality (particularly those of a legislative nature) has brought with it significant and widespread legislative and judicial pushback, often centering on the perception that such advances serve not to rectify longstanding systemic inequities but rather to offer unearned and unfair privileges to people of color. Using this lens, Weis et al. (2015) may have illuminated similar dynamics operating at the district, school, and/or educator level. While further research would be necessary to determine the extent to which and the ways in which this may be the case, it 
seems to me that the all-too-common failure of STEM equity-focused educational programs to gain traction is due at least in part to the implicit assumption that "we have already done enough." It is interesting to consider how this may play out at the network (i.e., district), organization, and individual level— the "we've done enough" mindset may not manifest in the form of direct resistance (by superintendents, administrators, educators, or others) to programs dedicated to addressing race-, ethnicity-, and genderbased STEM education gaps, but might instead cause education professionals to feel that simply offering these programs is sufficient. This possible explanation is particularly compelling in light of the importance of recognizing and responding to systemic dynamics that have the potential to affect the efficacy of STEM reform programs (Weis et al., 2015). If educators and administrators (consciously or unconsciously) accept the “we've done enough" mindset as valid, the need to make further modifications to practice and provide additional scaffolding to ensure the success rather than merely the existence of these programs is almost certain to be met with significant resistance.

In a similar vein, Morçöl (2010) stated in his examination of educational policy that "public policies are self-organizing systems that are constituted by the actions of self-conscious policy actors and they coevolve with other systems (natural systems and other policy systems)" (p. 53). This description aligns with the concept of nested systems noted by other complexity researchers (Byrne, 2005; Fenwick, 2012; McQuillan, 2008) and reflects the intersystem entanglements emphasized by Eoyang and Holladay (2013). The educational system cannot be considered as separate from adjoining elements of the landscape of United States politics and public policy, with the implication being that 
educational leaders who hope to generate meaningful policy change must address their efforts to a wide and varied range of potential stakeholders. In short, change and sustainable development in education, at whatever level, are not so much a consequence of effecting change in one particular factor or variable, no matter how powerful the influence of that factor. It is more a case of generating momentum in a new direction by attention...to as many factors as possible. (Mason, 2014, p. 6)

With this in mind, the tenets of complexity theory suggest planning for political action or policy change requires a period of thoughtful consideration regarding factors and agents impinging upon the issue one wishes to address.

Complexity theorists have argued — and I agree — that it is if not impossible, then certainly shortsighted, to understand any element of the educational process without recognizing and assessing the broader context and systemic factors at play; however, on its own, a systems-level exploration of learners as active agents is only part of the story. Researchers who employ complexity theory have not always been successful in identifying the ways in which a given system operates to privilege some individuals and groups at the expense of others (Byrne, 2005; Fenwick, 2012; Osberg \& Biesta, 2007). Fortunately, other theoretical frameworks exist that are explicitly intended to address such dynamics - by combining complexity theory with one of these, critical theory, a more comprehensive picture can begin to emerge.

Critical theory. While necessary, it is also insufficient to identify and map the ways in which complexity principles are evident in the context of my problem of practice; I chose to focus on this specific problem of practice due to deeply problematic 
disparities in STEM, and while complexity theory can help to elucidate the what, in this case it falls short in articulating the so what? For this, I turned to critical theory as a framework for interpreting what I earlier described through a complexity lens. Few if any previous studies have combined complexity and critical theory in this way; however, based on my understanding of these theories, I do not interpret this as an indication of poor fit but as a rich opportunity to build on the work of those who have come before.

In recent years, critical theorists (e.g., Basile \& Lopez, 2015; Mansfield et al., 2014) have problematized the very notion of the "STEM crisis" (i.e., the apparent underrepresentation of women and racial/ethnic minorities in STEM fields, to the detriment of diverse and innovative thinking). Basile and Lopez (2015), for instance, noted that federal reports advocating for inclusive STEM practices relied on arguments that "were made predominantly from a one-sided economic perspective, favoring the owners and operators of the STEM enterprise while humanitarian statements to create equitable access to and how Students of Color could themselves benefit from STEM access were virtually nonexistent" (p. 540). Such critical perspectives underscore the importance of questioning my own assumptions regarding the ways in which "adequate" vs. "underrepresentation" are structured and perceived, and by whom. Similarly, I am inspired by the work of Peralta et al. (2013), who attended sensitively and carefully to the role played by family and "community cultural wealth" in contributing to the development of individually meaningful and culturally relevant STEM identity. As I framed my problem of practice, I considered it fundamentally important that I never lose track of the humanity—and the agency — of learners, nor of the individuality and nuance of their identities and the reasons for their motivations and educational choices. 
As with complexity theory, critical scholars have also turned their attention to the examination of political processes, including the development, implementation, and evaluation of public policy. Indeed, there is a subset of applied critical theory-critical policy analysis - that has been defined as "a scholarly framework that understands policy as situated in specific contexts and their associated power dynamics" (Taylor, 1997). As with critical theory more broadly, critical policy analysis emerged as a counterpoint to the traditionally functionalist and "scientific" tradition of policy studies (Diem, Young, Welton, Mansfield, \& Lee, 2014), with scholars who engaged in critical policy analysis acknowledging their agency and positionality as researchers and using this as an entrée into the systems of power extant in policy discussions. Critical policy analysis has been employed as a framework in assessing a wide range of educational policies (Chase, Dowd, Pazich, \& Bensimon, 2014; Johnson \& Howley, 2015; Nordin, 2014), as well as the framing surrounding the current "STEM crisis" (Mansfield et al., 2014). Throughout these and the numerous other articles of scholarship produced by critical policy researchers, a common thread has emerged: The inclusion of a critical lens in educational policy studies permits the illumination of "the ways in which power operates through policy by drawing attention to hidden assumptions or policy silences and unintended consequences of policy practices" (Allan, Iverson, \& Roper-Huilman, 2010, p. 24).

Critique of theoretical framework. While the framework of complexity theory provides a powerful tool for exploring agentic learner dynamics within an entangled educational ecosystem, this theory has shortcomings. As a relatively new social science framework, with origins in a quantitative tradition, complexity theory is not designed to 
attend to the elements of interpersonal and intrapersonal lived experience educators must ultimately be concerned. Fenwick (2012) stated:

Important questions, perhaps particularly in considering professional education and practice, may seem invisible in complexity analyses. How does power flow within a system to enact particular entities, positions and rewards? What knowledge and activities, among the various relations and processes occurring within a complex system, are afforded the greatest visibility and influence over the movements and directions of the system? Whose interests are most advantaged or disadvantaged by the patterns that emerge? (p. 143)

In addition to suggesting critically important questions I was eager to explore through my study, these potential shortcomings of complexity theory necessitate deep consideration on the part of educational researchers considering the application of this framework to the study of learning and learners. It is, regrettably, not difficult to locate examples of studies in which dynamics of power and privilege are insufficiently addressed (Zellermayer \& Margolin, 2005) and even some of the most well-respected complexity theorists have posited that complexity theory "is more prone to regard the injustices of the world as inevitable consequences of complex dynamics" (Davis \& Sumara, 2008, p. 169). Fortunately, critical theory is perfectly suited to the illumination and assessment of precisely such dynamics, while simultaneously demonstrating certain gaps that are complemented by complexity theory.

With regard to these gaps, critical theory is, by definition, dedicated less to proving the generalizability of empirically generated findings than to "clarifying conditions of oppression, opening avenues of resistance, and refashioning liberating 
ideals" (Bronner, 2011, p. 5). It is troubling, then, that critical theorists often find themselves in an uphill struggle to initiate transformative modes of practice within the context of systems that privilege hegemonic epistemologies and applications of knowledge, or even to establish the theoretical groundwork necessary for such transformative practice to be enacted (Anderson, 1989; Aronowitz \& Giroux, 1985). At its core, critical theory is emancipatory in nature (Carlile, 2012; Duffy \& Scott, 1998); if a critical study fails to generate transformative action, destabilize hegemonic epistemologies, or at least provide direction for practitioners to engage in transformative work, it calls into question the meaningfulness of such a study. It is intriguing, then, to consider the fact that complexity theory offers a reconnection to a quantitative scientific tradition that has been to some degree deemphasized as a consequence of the questioning of positivist assumptions by qualitative researchers and theorists (Horn, 2008).

Taken individually, both critical theory and complexity theory provide a limited picture of the context and content of my problem of practice. Many of the critiques outlined earlier are equally valid when considering the application of these frameworks to educational policy and politics; however, I also call attention to additional limitations specific to this context. Young and Diem (2014) noted that "much of the critical policy literature is concerned with the policy being analyzed rather than the hows (i.e. different approaches for analyzing the issue) and whys (i.e. the various reasons for engaging in policy analysis) of policy analysis" (p. 1066). While the special issue they were introducing (focused on critical policy analysis) represented a valuable step toward addressing these shortcomings, I took their caution to heart, particularly given that it is not one that is naturally complemented through the pairing with complexity theory. By 
contrast, the limitation noted by Morçöl (2010) — that "complexity theorists will need to incorporate the accumulated insights of social theorists...into theirs to make meaningful contributions" (p. 59) to the scholarly examination of public policy-is well-aligned with the inclusion of critical theory. Nonetheless, Morçöl's caution is a reminder to seek an equitable (although not necessarily equal) balance between critical and complexity theory rather than allow one to overwhelm the other; it is through the weaving together of these distinct but equally valuable perspectives that the most meaningful insights are likely to emerge.

As I considered this weaving together, however, I also needed to acknowledge one final limitation of my theoretical framework, a limitation that was the inverse of what I believe to be a significant strength. Complexity theory and critical theory have been only rarely combined in the fashion I incorporate here, and while I believe this combination holds great potential for each to complement the weaknesses of the other, I recognized that I was also entering somewhat uncharted theoretical territory. This is not to say that scholars have entirely ignored the possibility of this pairing; from international relations (Cudworth \& Hobden, 2012) to Marcusian sociological studies (Garlick, 2011) to business and government (Alvaro et al., 2011; Bevan \& Gitsham, 2009), researchers have explored the relationship between complexity theory and critical theory in their particular fields. In educational research, however, such applications remain scarce. Firth and Morgan (2010), introduced and framed by Butt (2010), discussed the shared "openness" of critical theory and complexity theory, noting that "current discussion about quality in educational research and the movement towards 'evidence-based policy and practice' oversimplifies complex problems...Quality criteria do not sit outside of 
theoretical, methodological and philosophical perspectives" (p. 111). More recently, Cochran-Smith et al. (2014) employed complexity theory in conjunction with a critical realist frame to propose a path forward for teacher education research, positing that synthesizing complexity theory with critical realism deals with some of the central problems of sociological theory: a way to relate macro and micro issues without being reductionist and a way to describe the agency-structure relationship that accounts for human agency by acknowledging that human beings may have the capacity to initiate certain causal sequences. (p. 111)

Lastly, and once again beyond the borders of educational research (but highly relevant to my study), Marra (2015) paired complexity theory with critical feminist theory to consider approaches to evaluating gender equity from a public policy perspective. Such an approach, if taken on by policymakers themselves in addition to researchers, would represent a fundamental shift in schema for assessing the value and efficacy of policies and political acts, necessitate a sea change in the legislative and decision-making process, and potentially offer new opportunities for critical and transformative pedagogies.

Beyond the general scarcity of studies including a combined complexity and critical lens, an additional limitation of this framework relates to the disparate ontological and epistemological roots from which each theory originates. As noted earlier, complexity theory draws from a tradition steeped in positivism (or at least postpositivism), while critical theory, if not a paradigm unto itself as many suggest (Guba \& Lincoln, 2005; Mittwede, 2012; Ryan, 2018), is closer to constructivism or interpretivism than to positivism. This fundamental differing of paradigmatic perspectives is, I believe, the source of much of the complementariness between these 
theories, but also necessitates careful and honest attention on the part of the researcher to ensure that the resultant interpretations are valid, resonant, and representative of both theories. I found it interesting that this point was not, to my mind, raised in any meaningful way in the studies I have cited that combine critical and complexity theory, but did not consider this justification for me to disregard its importance in considering my own approach to planning and interpretation.

Reflections on theoretical framework. Through my work, I grew increasingly aware of the ongoing tensions, particularly at the federal level, regarding budget appropriations for key ISE funding channels (e.g., NASA, the Institute for Museum and Library Services [IMLS], the NSF, the National Institute of Health, and the National Endowments for the Arts and Humanities). While such programs - in contrast to, for example, military expenditures — have historically been called upon to justify their existence with disheartening regularity, it is even more distressing to note that the presidential budget proposal for the 2017 fiscal year recommended the wholesale elimination of several of those funding channels named above (Price, 2017). Compounding this state of affairs are the federal restrictions regarding the ways in which and the extent to which the United States government permits nonprofit educational organizations to engage in political advocacy and lobbying. Organizations like OMSI run the risk of sacrificing their tax-exempt status if their actions stray into the realm of "directly or indirectly participating in, or intervening in, any political campaign on behalf of (or in opposition to) any candidate for elective public office" (Internal Revenue Service, 2016, paragraph 1). Nonprofit educational organizations are legally permitted to take a public position on policy issues and may participate in lobbying activities 
(Afterschool Alliance, n.d.), but must tread carefully to avoid inadvertent transgressions with potentially devastating consequences.

When viewed through the dual theoretical frameworks outlined above, tensions such as these illustrate both the principles of complexity and the unavoidable influence of unequal distribution of power and privilege across groups and individuals within the systems of educational policy. Politics and policy intersect in complex ways with the use and misuse of power at multiple systems levels; for example, some funders explicitly limit disbursement of funds to projects focused on specific topics at the exclusion of others (an illustration of the explicit exercise of power, per Fowler, 2014). Furthermore, based on my personal observations and reflections, I posit that the consistent experience of navigating these various funding channels has the potential to implicitly inform customs, norms, and procedures within educational institutions. I approached my problem of practice from the personal perspective of an informal education researcher, and when the framing is constrained to informal education the most salient system of policy and politics is at the federal level (due to current funding structures). However, it was impossible to understand my problem of practice using such a tightly focused lens (particularly bearing in mind the tenets of complexity theory), and when I broadened my view to include even the formal education system at both the $\mathrm{K}-12$ and higher education level, I found that both state and local levels of government become far more relevant for consideration. At the federal level, as noted previously, OMSI and other ISE institutions are often beholden to funding sources and structures established and managed by various federal agencies, including but not limited to NSF, NIH, NASA, and IMLS. As these are federally-managed agencies, their budgetary appropriations and grant funding criteria are 
prone to fluctuations concurrent with changing administrations and priorities; these fluctuations in turn affect the types and degree of projects pursued and audiences served. State government, meanwhile, plays a key (although not exclusive) role in determining the ongoing viability of public colleges and universities, as does local government with regard to K-12 school districts; all of these colleges, universities, and school districts represent important elements of the STEM education ecosystem, with the result being that the influence of government at every level can be felt by those within the ecosystem.

This is not to say that any one level of government is necessarily more or less important for consideration—on the contrary, an examination of learner motivations and dynamics within an ecosystemic framework necessitates a holistic understanding of governmental influence — but the specific relationships is complex rather than straightforward. Having said this, one way some consistency may exist is in the form of resource scarcity and the enactment of power within and between the agents who constitute and co-navigate these systems. Whether in the form of competition between museums, universities, and other organizations for federal grant funding; colleges and universities negotiating funding at the state level; or the perpetual and widespread underfunding of the public school system, these limitations have significant ramifications for the leadership, direction, and experience of education in Portland and elsewhere across the country.

Lastly, in reflecting on the ways in which the issue of STEM inequities has been approached, particular by federal agencies, I was reminded of Basile and Lopez' (2015) analysis of federal education policy briefs wherein they problematized the framing typically employed in considering and discussing the underrepresentation of racial and 
ethnic minorities in STEM fields. Basile and Lopez argued that the very real issue of inequitable representation in STEM tends to be approached by federal agencies (including those, such as the NSF and NRC, responsible for publishing widely-distributed reports) using essentializing and reductive terminology, and is generally presented from an economic perspective primarily benefitting those who already possess power in STEM enterprise. Mansfield et al. (2014) supported this position in their feminist critical policy analysis of the discourse surrounding the "STEM crisis." Mansfield et al. found that from the mid- $20^{\text {th }}$ century on, discussion of underrepresentation in the STEM fields at the governmental policy-setting level has been largely influenced by (and designed to serve the interests of) private and industry stakeholders. The authors supported this assertion through a thorough examination of federal and state documents, historical and contemporary, as well as statistical informal and policy documents.

I am not of the opinion that such framing is by and large the result of intentionally exploitative thinking on the part of policy actors; while the occasional exception likely exists, my suspicion is that these reports and related policies are more often generated by well-meaning individuals and groups who truly wish to address STEM inequities. If nothing else, however, the framing and definition of this issue serve as an example of interest convergence (Taylor, 2016), the phenomenon wherein the interests of oppressed or underserved groups are advanced only insofar as dominant groups stand to benefit by such advancement. Equally importantly, these examples underscore not merely the appropriateness but the urgency of taking a critical complexity approach to exploring my problem of practice. I posit that it is crucial to recognize that "a critical policy analysis approach highlights how policies can fail to provide adequate provision for students with 
diverse learning needs, and how the broader conditions within which such policies are developed play through and influence how they are discursively constructed" (Hardy \& Woodcock, 2015). Taken together, these perspectives permit us to speak to the complexity and nuance of STEM learner experiences.

Reflecting on the theoretical analysis outlined above while simultaneously recognizing the critiques of both complexity and critical theory, three key implications emerge for my study. First, while I approached my problem as an informal education professional, I needed to take the tenets of complexity theory to heart and recognize the importance of attending to the broader system in which my organization is situated and through which individuals chart their STEM learning journeys. As I considered inequitable rates of visitation to informal learning, I needed to dig deeply, searching beyond surface-level causes to explore the hidden entanglements between agents and systemic factors. Second, the application of critical theory provided a vital reminder that neither the problem I identified nor the attention I dedicated to its examination were value-free. As with the previous point, rather than, for example, assuming that differences in motivation and self-efficacy are simply the result of individual variation, it was necessary to push past such straightforward answers to ensure hidden barriers and supports were brought to light. Lastly, the pairing of complexity theory with a critical lens sensitized me to the ways in which these frameworks intersect. The characteristics of complex systems cannot and should not be considered simply "the way things are (or will be)" but instead contribute to the strengthening or weakening of the power dynamics underlying inequitable representation in STEM fields; by the same token, a critical theory approach to my study would be incomplete in light of the myriad nested and "massively 
entangled" (Eoyang \& Holladay, 2013, p. 17) subsystems and agents present in the Portland STEM learning ecosystem.

\section{Review of Research Literature}

Moving on, then, from a demarcation of the theoretical framework that serves as a lens for my study, the following pages outline the concepts forming the core of my exploration of learner motivations and attitudes, recognizing that learners' choices in navigating their STEM ecosystems are strongly influenced by their motivations, attitudes, and experiences regarding STEM. In addition to the variables introduced in my research questions - including self-efficacy, STEM ecosystems, culturally sustaining pedagogy, and the six strands of science learning - it was also necessary to review the literature trends of inequitable representation of women and many communities of color in the STEM fields, both to establish the existence and persistence of gaps and to critically examine the narratives that surround these trends in the popular and academic press. I posit that these concepts relate in ways that are complex and, in many cases, not yet fully understood; indeed, the six strands of science learning and the STEM Funders Network's (2016) conceptualization of STEM ecosystems are minimally represented in the literature in any way, let alone in conjunction with self-efficacy or culturally sustaining pedagogy. While the landscape is not entirely barren, the general dearth of scholarship in these areas suggests an additional, if ancillary, benefit that may accrue through my study.

STEM field inequities. Given the dynamics of power, privilege, and oppression present in the United Stated educational system, the motivations, attitudes, and experiences of STEM learners are likely to be fundamentally affected and shaped by their race and gender. In Malcom's (2010) study, which provides a compelling illustration of 
the importance of a critical frame in assessing STEM learning and engagement, she provided a description of the educational problem of practice she intends to address through her study, as well as a clear and compelling articulation of the legitimacy and urgency of this problem. Specifically, Malcom noted three key facts germane to her position: That two million new professionals will be needed in the STEM fields to replace the baby boomers expected to retire in the coming years, that Latina/os comprise only $4.3 \%$ of the STEM workforce, and that one-half of all school-aged youth in the United States are Latina/o. Based on this justification, Malcom conducted a study exploring the institutional pathways, financial strategies, and effects of student debt burden experienced by Latina/o STEM baccalaureates, employing a quantitative research methodology relying upon secondary analysis of an existing survey design-generated dataset (the 2003 National Survey of Recent College Graduates available from the National Science Foundation).

Among the key findings of Malcom's (2010) study were that a sizeable number (61\%) of Latina/o STEM baccalaureates had attended community college at some point, with roughly one-third ( $18 \%$ of the overall sample) having earned an associate's degree prior to their bachelor's. Interestingly, nontraditionally aged students- those 25 years or older —were disproportionately overrepresented among associate's degree holders, and students whose parents had not earned a bachelor's degree were disproportionately likely to complete an associate's prior to earning their bachelor's. Malcom further noted the problematic implications of earning an associate's degree, including perceptions of community colleges as lower-status than four-year universities and the resultant challenges in gaining entry into such "prestigious" institutions subsequent to the 
completion of an associate's degree. Malcom's findings provided some evidence for this claim, as Latina/o STEM baccalaureates who first earned an associate's degree were less likely than those who did not earn an associate's degree to go on to complete their bachelor's studies at institutions classified as highly competitive (i.e., institutions to which a relatively small percentage of applicants are accepted, and which are resultantly perceived as particularly selective and desirable), private universities, or research universities. Bearing these findings in mind, the two primary directions for educational researchers and practitioners Malcom identified at the conclusion of her analysis were (a) the modeling of community college pathways to STEM and (b) further identification of barriers to transfer access and transfer student success.

I found it interesting to consider the study conducted by Malcom (2010) through an analytical lens provided by the work of Fouad and Santana (2017). Fouad and Santana's analysis of research findings focused on past work that employed a framework of social cognitive career theory in assessing the STEM career pathways and barriers experienced by women and racial and ethnic minorities, and they would likely hasten to point out the importance of self-efficacy as a potential mediating or moderating variable at play in Malcom's study. Fouad and Santana noted that although Mexican American middle school girls expressed greater perceptions of social supports for STEM involvement than did boys from the same age group and cultural background, boys in their study nonetheless expressed a higher level of self-efficacy related to math and science skills did girls. Findings such as this complicate the relationship posited by Malcom between family educational history and STEM education pathways, as Malcom's study emphasizes the role of the baccalaureate status of a student's parent(s) 
while disregarding student sex or gender as a factor for consideration. I admit I have no direct experience with the NSRCG instrument or dataset and it is possible that these variables are not included, but I found this highly unlikely; it may be that Malcom had to make difficult decisions regarding which variables to include and exclude from analysis. The example cited above regarding self-efficacy and perceived social supports is only one of many that could be drawn from Fouad and Santana's findings to illustrate the variability of STEM education pathways and the necessity of acknowledging and valuing the intersectionality of learner identities. Both Malcom's (2010) and Fouad and Santana's (2017) studies speak to the legitimate realities of individuals' lives, and through both what is included and what is excluded, serve to emphasize the importance of bringing multiple lenses to bear on the exploration of so complex a story.

Related this point, in their recent - and deeply problematic - analysis of the persistent gender-based gap in representation in the STEM fields, Wang and Degol (2017) identified and summarized six overarching explanations that have been posited regarding the underrepresentation of women and girls. Across their meta-analysis of existing studies and meta-analyses, the six explanations articulated by Wang and Degol as being "empirically supported" include (a) differing levels of cognitive ability between women and men; (b) relative rather than absolute cognitive strengths among men and women; (c) general career preferences held by women and men; (d) the varying degrees to which lifestyle values and concern for a family-work balance were expressed by men and women; (e) beliefs regarding field-specific abilities, including possession of fixed versus growth mindsets of intelligence; and (f) overt and covert gender-based biases and stereotypes. Troublingly, rather than critically interrogating these explanations, Wang 
and Degol largely accepted them on the basis of their establishment within the body of extant literature and posited that the underrepresentation of women in STEM career fields is the result of interplay between these six factors and cannot be fully understood through a lens of singular causality. Taking these six factors together, Wang and Degol provided a number of recommendations for educational policy and practice with the goal of improving outcomes for female students and workers in STEM fields. Wang and Degol's recommendations do not necessarily appear to be constrained to formal education settings - for example, their suggestion that interest enhancement should be considered a focus alongside the enhancement of ability is well-aligned with informal STEM education contexts. Similarly, the authors encouraged practitioners and policymakers to dedicate energy to the early cultivation of STEM interest and to focus on breaking down gendered stereotypes regarding STEM, cultivating growth mindsets of intelligence, incorporating storytelling into STEM education, emphasizing the real-world relevance of STEM degrees, supporting the visibility of female role models in STEM fields, and building in structures to ensure women are accommodated in the workplace.

While Wang and Degol's (2017) recommendations are doubtless wellintentioned, they are difficult to read without being reminded of Basile and Lopez' (2015) analysis of federal education policy briefs wherein they problematized the framing typically employed in considering and discussing the underrepresentation of racial and ethnic minorities in STEM fields. At the crux of Basile and Lopez' argument is the notion — supported by their findings - that the very real issue of inequitable representation in STEM tends to be approached by federal agencies (including those responsible for publishing widely-distributed reports) using essentializing and reductive 
terminology, and is generally presented from an economic perspective primarily benefitting those who already possess power in STEM enterprise. While Wang and Degol focused on gender rather than race and ethnicity, I would suggest that these critiques could be considered equally valid in the context of their analysis; I reviewed their article multiple times and found it interesting that they employed "traditional" binary definitions of gender (conflated with sex) without questioning the appropriateness of such definitions. Likewise, Wang and Degol appeared to take for granted that equitable STEM participation is desirable while providing no justification for this stance-I will note that I wholeheartedly agree with this position insofar as all learners should have equitable access to STEM opportunities, but also feel it is both necessary and relatively straightforward to provide an articulation of why the current state of affairs is problematic. Without specifying the reason(s) for conducting an examination of systemic dynamics underlying the race-, ethnicity-, and gender-based underrepresentations that continue to occur in STEM fields, it is all too easy to slip into the default framework that favors STEM owners and operators (a perfect example of interest convergence in action!).

In their 2015 study, Barth, Guadagno, Rice, Eno, Minney, and the Alabama STEM Education Research Team approached the question of gender-differentiated career interest using a three-part theoretical framework to assess the interplay of masculine or feminine stereotyping of occupations with occupational affordance of gender-based goals. Barth et al. predicated their study on the position that increasing the presence of women in STEM fields will contribute to addressing the shortfall of qualified workers in these fields. In alignment with the precepts of Social Role Theory, Role Congruity 
Theory, and Precluded Interest Theory, Barth and colleagues hypothesized that (a) the career interests of both men and women will be affected by occupational gender stereotypes, (b) occupational preferences will differ between women and men based on each occupation's accommodation of communal versus agentic roles respectively, and (c) occupational preferences will be affected by a combination of gender, occupational stereotypes, and goal affordances. On this basis, Barth et al. conducted a survey of undergraduate STEM students at two time points ( $N=186$ at first time point, $N=200$ at second time point, $n=148$ retained) using the Life Goals and Gender Stereotypes (LGGS) instrument developed specifically for this study. The results of their analysis indicated partial support for the first of their hypotheses at the first time point (with male participants demonstrating a strong preference for masculine-coded jobs but female participants indicating no particular preference), with the hypothesis fully supported at the second time point. Their second hypothesis - that occupational preferences would differ between women and men based on each occupation's accommodation of communal versus agentic roles — was unsupported at either time point. Indeed, female respondents indicated a stronger preference for occupations affording salary goals than those affording helping or family goals, in exact opposition to the hypothesized relationship, while the third hypothesis (that occupational preferences would be affected by a combination of gender, occupational stereotypes, and goal affordances) was partially supported. In discussing the implications of their study, Barth et al. note that given the nature of the sample, their findings indicate that women enrolled in STEM courses may not be discouraged from STEM careers solely on the basis of male stereotypes but that male students may have an aversion to female-stereotyped careers. Lastly, Barth et al. 
note that their findings would appear to suggest that at least during their early college years, women may not be as concerned with the types of goals typically associated with femininity (e.g., prosocial orientation, family-friendliness) as past research has suggested (Ceci, Williams, \& Barnett, 2009; Diekman, Brown, Johnston, \& Clark, 2010; Diekman, Clark, Johnston, Brown, \& Steinberg, 2011; Eagly, Wood, \& Diekman, 2000; Evans \& Diekman, 2009), although such goal orientation may change over the course of an individual's life.

In considering Barth et al.'s (2015) findings and interpretation, I was reminded of the arguments made by Mansfield et al. (2014) in their feminist critical policy analysis of the discourse surrounding the "STEM crisis." At the crux of the position taken by Mansfield et al. is that from the mid- $20^{\text {th }}$ century onward, discussion of underrepresentation in the STEM fields at the governmental policy-setting level has been largely influenced by (and designed to serve the interests of) private and industry stakeholders, including higher education and IT companies. Mansfield and colleagues supported this assertion through a thorough examination of federal and state documents, historical and contemporary, as well as statistical informal and policy documents from such agencies as the United States Department of Labor, and regrettably, the framing employed by Barth et al. does nothing to contradict such claims. This is particularly troubling given that the current dominant frame of discourse is by no means immutable; Mansfield et al. noted three key recommendations for questioning and destabilizing the hegemonic narrative that has thus far tended to prevail. First, Mansfield et al. called for a disruption of the privileging of a traditional Western conceptualization of what constitutes "knowledge" and "science," an approach that could potentially have been 
taken by Barth et al. had they questioned the standard categories of STEM occupations. Second, any conversation of STEM education inequities must include a thoughtful and thorough consideration of the complex interrelationships between multiple categories of power, privilege, and identity inhabited and navigated by every learner, a point that was not explored by Barth et al., who focused instead on gender as the sole variable of importance. Lastly, Mansfield et al. posited that schools, universities, and many fields of study can accurately be characterized as bureaucratic and hierarchical, with these systems and structures operating to preserve a status quo that restricts the growth and development of many while supporting very few, a dynamic that remained unchallenged by Barth et al. These points, while not comprehensive, are vital to consider in any discussion of STEM inequities; I am grateful for the critical lens offered by Mansfield et al. and intend to continue striving for a nuanced and counterhegemonic framing in my conceptualization and examination of my problem of practice, including but not limited to in the study presented in this document.

Self-efficacy. Rather than considering them passive consumers of information, educational practitioners and researchers must recognize STEM learners as thoughtful, critical, active agents in charting their educational journeys. Emerging from social cognitive theory (Bandura, 1971, 1992, 2006, 2011; Bandura \& Locke, 2003), the concept of self-efficacy (Bandura, 1977; Coutinho \& Neuman, 2008; Huang, 2016; Jiang et al., 2014) is at heart "concerned with judgments of how well one can execute courses of action required to deal with prospective situations" (Bandura, 1982, p. 122). Building upon Bandura's (1986b) work regarding self-efficacy and Marsh's (2007) concept of self-identity, Parker, Marsh, Ciarrochi, Marshall, and Abduljabbar (2014) dedicated their 
study to the disentanglement of these concepts and the disambiguation of the relationship of each to achievement in mathematics. Parker et al. began by briefly outlining the conceptualizations and extant literature surrounding self-efficacy and self-concept, followed by a comparison and contrasting of these variables. Parker and colleagues noted that based on the results of several meta-analyses, the vast majority of past research studies involving self-efficacy and self-concept appear to include only one rather than both, making an examination of the relationship between the two difficult. To address what they perceive as a key shortcoming in the current body of knowledge, Parker et al. conducted secondary analysis of a large-scale existing dataset (the 2003 Longitudinal Study of Australian Youth, or LSAY; $N=10,370$ ). This dataset included relatively equal proportions of female and male participants; while Parker et al. provide additional demographic information, I am not sufficiently familiar with the broader demographics of Australia to confidently speak to the representativeness of the sample on the basis thereof. Analysis of the measures included in the study provided a wide range of intriguing findings, including that self-concept was more domain-specific than selfefficacy, and that mathematics self-efficacy significantly predicted university entry (not restricted to STEM) while self-concept was a significant predictor of STEM course selection. Parker et al. emphasized that these findings provided evidence of the relationship between self-concept and self-efficacy while simultaneously highlighting the importance of considering them as distinct from one another, and recommended further research to continue the exploration of these nuances.

Considering the ramifications of Parker et al.'s (2014) approach and findings for my study, I was drawn to consider Delgado Bernal and Villalpando's (2016) illustration 
of the importance of alternate narratives to explain observed outcomes. In the case of the study conducted by Parker et al. (2014), while the researchers did not focus on experiences of faculty or learners of color, a subversive reading of the findings was nonetheless possible. According to Parker and colleagues, "When an academic outcome is heavily based on progression... descriptions of competence like those found in selfefficacy may be more important...For outcomes which depend primarily on choice between academic domain options, however, self-concept may be more important" ( $\mathrm{p}$. 44). An alternate narrative to reframe these statements to address the perspectives of students of color might read something like the following: "Learners of color-whose experiences were not analyzed separately from White learners in this study—have been consistently shown to receive a systematically lower level of guidance and support from teachers and peers, particularly with regard to STEM topics (including mathematic). Given this fact, an exploration of self-assessment would be incomplete without acknowledging the importance of the moderating variable of race; indeed, it should be emphasized that defining matriculation into a STEM field as a 'choice' potentially downplays the significance of race as a defining characteristic of learners' lived experiences, for choice and agency can look and feel very different on this basis." Having said this, I feel it necessary to note again that Parker et al. conducted this study using data collected from Australian students, and the dynamics of race are likely to differ in some regards, perhaps large and perhaps small.

Culturally sustaining pedagogy. The idea of culturally sustaining pedagogy emerging less than 10 years ago as a conceptual "next step" to the approach of culturally relevant pedagogy described by Gloria Ladson-Billings (1995), who has since lauded it 
as a "remix" offering deep potential for advancing the field of education (LadsonBillings, 2014), Django Paris (2012) proposed the idea of culturally sustaining pedagogy in recognition of the need for an educational framework that both foregrounds the legitimacy of learners from diverse cultural and linguistic backgrounds and equips learners with the tools necessary to navigate dominant cultural systems. As described by Paris in his foundational article:

The term culturally sustaining requires that our pedagogies be more than responsive of or relevant to the cultural experiences and practices of young people - it requires that they support young people in sustaining the cultural and linguistic competence of their communities while simultaneously offering access to dominant cultural competence. Culturally sustaining pedagogy, then, has as its explicit goal supporting multilingualism and multiculturalism in practice and perspective for students and teachers. (p. 95)

Importantly, culturally sustaining pedagogy also offers a platform for a caring and growth-oriented critique of other asset pedagogies, something that is perhaps not altogether absent from but certainly scarce within the current body of educational research. Paris and Alim (2014) noted that, for example, the framing of pedagogy as culturally relevant does not necessarily ensure that the knowledge and practices in question are legitimized or maintained. Likewise, these authors urge for a temporal reorientation of focus, from a past-oriented perspective focusing primarily upon cultural traditions and heritage (heritage practices) to a more balanced perspective that incorporates a recognition and valuing of contemporary knowledge and ways of being (which they define as community practices). Lastly, Paris and Alim urged practitioners 
and scholars who take up the work of culturally sustaining pedagogy to employ this framework "to support the practices of youth and communities of color while maintaining a critical lens vis-à-vis these practices" (p. 92). Rooted in this foundation, culturally sustaining pedagogy offers a powerful avenue forward for educators, researchers, and learners to engage in co-construction of knowledge and understanding that celebrates and sustains cultural plurality while neither granting a place of primacy to the dominant cultural worldview nor considering as beyond critique one's own practices and biases.

In the years since its introduction as an educational framework, culturally sustaining pedagogy has been employed in the examination of the role of tribal sovereignty in the schooling of Native American students (McCarty \& Lee, 2016), the self-directed exploration of Latinx students' names and cultural and familial naming practices (Nash, Panther, \& Arce-Boardman, 2018), the selection and utilization of informational texts in classrooms (Kganetso, 2016), and the use of hip-hop in youth cultural organizing to facilitate arts-based civic engagement (Kuttner, 2016), among other contexts. As with most of the components of my conceptual framework, the majority of the (admittedly limited) usages of culturally sustaining pedagogy in published research have taken place within formal learning settings; however, at least one exception to this rule does exist. Weiland (2015) conducted a phenomenological study of Hispanic mothers' experiences in an informal science center, particularly focusing on the degree to which the informal STEM learning setting facilitated multilingual and multicultural engagement and provided sustaining and legitimizing connections to participants' cultural backgrounds and lived experiences. The results of Weiland's study indicated that across the eight participants, all of whom had recently immigrated to the United 
States from Latin America, four key themes were evident. The families (or at least the mothers) were largely unfamiliar with, and in some cases uncomfortable in, the environment of museums; additionally, their experiences in the science center afforded few opportunities for equitable cultural and linguistic access and engagement when compared with United States-born, native English-speaking visitors. Several of the mothers interviewed in the course of the study mentioned an appreciation for the lessstructured, free-choice learning tools and experiences available to their children in the science center, with one participant noting that "In school they don't do these kinds of things, they do American things. I don't know what they are exactly, but they are American things" (Weiland, 2015, p. 98). However, for several participants, this enthusiasm was tempered by their acknowledgement that they felt poorly equipped to support and engage with their children in this learning setting due to their (self-perceived) lack of science knowledge, the absence of environmental scaffolding for family engagement, or both. While this study did provide some evidence of the potential held by science centers and museums to engage with visitors in a culturally sustaining and counterhegemonic fashion, it also highlighted many of the ways in which institutions like OMSI have so far fallen short of achieving this potential. While OMSI has engaged in work that aligns with such tenets of culturally sustaining pedagogy as support of cultural pluralism through legitimization of multiple ways of knowing and co-development of learning experiences with diverse communities (OMSI, 2017; Roots of Wisdom Project Team, 2016), the museum has also repeatedly stumbled. The OMSI staff recognize they have far to go in weaving these principles into the very fabric of our organization. 
As noted by Paris (2016), while the widespread cultural and demographic shifts taking place across the United States have brought unprecedented opportunities for enrichment of learning and representation of diverse voices, they have also elicited immense and troubling backlash against minoritized learners and communities. The events occurring in the United States at the time of this writing are both powerfully uplifting and deeply disturbing, and in both counts represent a clear imperative for STEM educators of all stripes to, as Doucet (2017) proposed, (a) increase their knowledge about diversity, (b) built communities of trust in their learning spaces, (c) involve families and communities of learners, (d) combat prejudice and discrimination, (e) address the full complexity of diversity, and (f) promote global perspectives of and through education. Whether through such approaches as youth participatory action research (Walsh, 2018), narrative analysis (Puzio et al., 2017), or the critical quantitative methodology I employed in my study, it is incumbent upon educational researchers to engage in culturally sustaining work that both supports and builds upon the efforts and experiences of practitioners.

STEM ecosystems. As I have argued earlier, in spite of educators' generally heartfelt commitment to supporting interest, engagement, and the development of content knowledge, the process of holistic STEM ecosystem navigation by learners remains poorly understood. In a study intended at least in part to bridge this gap, Falk and colleagues (2016) sought to address what they identified as an absence of large-scale comprehensive datasets regarding the role of science centers in providing accessible and engaging STEM (science, technology, engineering, and math) learning experiences. The team of 20 researchers employed a quantitative study methodology and a survey design 
method to collect data from an international sample of 6,089 participants (including both "users" of science centers and individuals who were not categorized as such) representing 13 countries. Falk et al. (2016) focused their examination on a total of three independent variables, including how recently participants had visited a science center, as well as the nature of their experiences and the "dosage" (essentially, the duration) of their visit; these variables were paired with seven dependent measures assessing STEM knowledge, interest, participation (or behavior), and identity. Inferential analyses of these data included parametric and nonparametric univariate and bivariate statistical tests, and the researchers conducted both reliability assessments (specifically of Cronbach's $\alpha$ ) and exploratory factor analysis of scales constructed from multiple questionnaire items. The findings of this study indicated that a positive correlation existed between science center visitation and a number of the dependent variables identified above - a few of particular note being STEM interest and curiosity, participation in free-choice STEM leisure activities, and a sense of STEM identity—and that greater dosage tended to correlate with stronger statistical relationships.

The connections between this study and my own area of inquiry were myriad, ranging from the researchers' overall objectives to the findings generated by their analyses to the design of the study itself. In terms of objective, I welcomed a deeper understanding of the role played by science centers in STEM knowledge, interest, and identity development, and while I do not believe that Falk and colleagues suggest that their study was inclusive of all possible avenues of examination, it represented one valuable foray into a landscape I hope to explore. Regarding findings, Falk et al. may suggest that science center visitation does indeed correlate with a multitude of relevant 
attitudinal and behavioral outcomes, but what of causality? Further, and equally if not more important from my perspective, how do science centers fit into and complement (or not) the other educational resources available in their local STEM learning ecosystems?

Past research has clearly established that even within the same STEM education environment, learners may seek and experience different learning outcomes due to individual motivations, attitudes, and racialized/gendered identities (Ceci \& Williams, 2011; Hanson, 2004; Hardin \& Longhurst, 2016; Jones et al., 2000; Peralta et al., 2013). However, I also posit that through their decentralization of the typical locus of pedagogical control from settings established as for learning to encompass locations such as the home, afterschool and out-of-school contexts, and other potential sources of personally resonant experiences, STEM education ecosystems almost by definition offer a culturally sustaining approach to learner engagement, a position similarly held by Lee (2017).

The six strands of science learning. While frameworks for categorizing and understanding learner impacts across formal and informal STEM education settings are rare, the six strands of science learning offered a point of connection between both of these settings and the motivations, attitudes, and experiences of learners. Developed through a large-scale examination of science learning in informal education settings (NRC, 2009), the six strands of science learning include four capabilities (strands 2-5) that were originally posited in an earlier report addressing $\mathrm{K}-8$ formal science learning (NRC, 2007), supplemented by two additional capabilities (strands 1 and 6) that are of particular importance in ISE contexts. The six strands framework has been applied to studies of teacher preparation (e.g., Avraamidou, 2015; Crowl, Devitt, Jansen, Zee, 
\&Winograd, 2013), and has been embedded in reports, symposia, and other dissemination outputs within the ISE field (e.g., Cody, 2010; Krishnamurthi \& Rennie, n.d.). As Allen (2004) noted, many science center attendees conceptualized their visits as leisure time rather than solely dedicated to learning. Interestingly, in their landmark Synergies study, Falk et al. (2016) explicitly operationalized science center visitation as distinct from "free-choice science and technology-related leisure experiences (e.g., reading science and technology-related books and articles or watching science and technology-related media)" (p. 851). While Falk and colleagues provided no explanation for this distinction, the reliability of the "free-choice STEM-related leisure activity" scale was acceptable (Cronbach's $\alpha=.78$ ), indicating some degree of conceptual integrity. In contrast, Tunnicliffe (2008) conceptualized school group visits as being learning-focused and family visits as constituting free-choice leisure time.

This apparent lack of consensus—coupled with, again, what appears to be a reliance on educators' perceptions rather than direct engagement with learners to determine how they, in fact, conceive of their decisions to visit or not visit science centers — could also begin to be explored through my study. For a number of structural and pedagogical reasons, different parts of the STEM ecosystem are more or less readily able to provide opportunities for different categories of learning outcome. While it may not be sufficiently robust to provide a stand-alone approach to guide this study, the six strands of learning framework has the advantage of being one of the few to be developed explicitly to contribute to an understanding of ISE settings and learners. The six strands framework also offers a bridge between the other theories I intend to bring to bear on my research problem. With this in mind, the six strands may offer a useful deductive schema 
for interpreting the ways in which learner goals, self-efficacy, and agentic navigation of STEM ecosystems map (or fail to map) to key STEM education outcomes.

Critique of research literature. A limitation of this research literature relates to (a) the paucity of evidence regarding the validity and meaningfulness of the STEM ecosystems framework from the perspective of learners, (b) the rarity of usage of the six strands of science learning as an explicitly-articulated framework in academic research, and (c) the relatively inchoate nature of culturally sustaining pedagogy as an approach to understanding and engaging in education in general and STEM education in particular. With the exception of Crowl et al. (2013), who employed the six strands as an interpretive framework in much the same way as I do in my current study, in peerrefereed journal articles this framework is generally mentioned in passing if at all (e.g., Sample McMeeking, Weinberg, Boyd, \& Balgopal, 2016). Given the use of the six strands framework over the years at OMSI and elsewhere in the ISE field (Avraamidou, 2015; Hudson, Duncan, \& Reeve, 2015; Krishnamurthi \& Rennie, n. d.), a more integral inclusion in a robust research context is past due, but it must be acknowledged that the absence of scholarly literature from which to draw provides me with few signposts to guide my own implementation. Additionally, while the National Research Council developed the six strands framework with ISE settings specifically in mind, one significant weakness of this framework is the fact that seemingly no assessments of cultural specificity or generalizability have yet been undertaken. Understanding the importance of educational contexts that value and sustain the culturally-specific approaches and expectations of learners (Alim \& Paris, 2017; Paris, 2012) and the ways the U.S. education system acts as a mechanism for the reification of White supremacy 
while devaluing other cultures' learning practices (Gillborn, 2016), it is vital that the theoretical and practical advances made by informal educators be critically examined for exclusionary implications. Acknowledging this caveat, these and other prior studies do offer valuable contributions to educators' understanding of learning in formal and informal settings; however, none apply the six strands framework to explore the implications of the motivations underlying individuals' (conscious or unconscious) decisions to engage with some resources while dismissing others within their available STEM ecosystems. In conducting such an exploration, it is vital to recognize the role of various categories of privilege in affording or constraining access, real or perceived, to STEM learning opportunities.

Unlike the six strands of science learning, to the extent that the framework of culturally sustaining pedagogy has taken root to date, it has done so primarily within the context of formal educational practice and research. With the exception of Weiland's 2015 study of Latina mothers' experiences in a science museum as culturally sustaining (or not sustaining), no literature yet exists that describes the application of culturally sustaining pedagogy to ISE. This is not necessarily to say that culturally sustaining work has not been undertaken, only that it remains either unpublished or not named as such; in any case, however, my study offered an opportunity to extend and deepen the out understanding of culturally sustaining dynamics in informal learning settings and other areas of learners' educational ecosystems. I also noted that, somewhat in keeping with the inherently critical nature of culturally sustaining pedagogy, very few studies have paired this framework with a quantitative methodological approach. Indeed, with the exception of a single dissertation (Blalock, 2013) wherein the author conducted 
secondary analysis of quantitative data from the National Indian Education Study using structural equation modeling, I was unable to locate any examples of quantitative explorations of culturally sustaining pedagogies. This, again, placed me in a position of having minimal prior literature upon which I could rely in charting my methodological path through this conceptual terrain, and while I recognized the challenges this may present, I was excited to have the opportunity to forge new paths and perhaps offer new learning and tools to contemporary and future researchers. Lastly, I feel compelled to note, as highlighted by Puzio et al. (2017), that true engagement in culturally sustaining pedagogical practice (and, I would posit, research) is a process that brings with it great vulnerability and potential for mistakes and missteps. I entered into this work with humility and a recognition of the vast degree of learning that I had yet to do from my colleagues, partners, and participants, and to balance a forgiveness of my inevitable shortcomings with an awareness of the critical importance of cultural sustenance in learners' everyday lives and experiences.

Similarly, while the idea of STEM learning ecosystems has been discussed with some regularity since the establishment of the STEM Funders Network (Fleet Science Center, 2018; Journal Staff, 2017; Mincarelli, 2015; Southern California Grantmakers, 2016), only a very small number of studies have been published that employ this framework (Bevc, Young, \& Peterman, 2016; Corin, Jones, Andre, Childers, \& Stevens, 2017). Clearly the field stands to benefit from further empirical validation of this framework from the perspective of learners, those who actually experience and navigate STEM ecosystems in the course of their educational journeys; however, I was also necessarily limited in the claims I could comfortably make at the outset of my study 
regarding the veracity of the ecosystemic framework I intended to use. The limitations I have identified here were not sufficient to cause me to question the inclusion of these concepts in my study, only to sharpen my attention to detail and emphasize the importance of critical thinking throughout the course of the project.

Regarding the more well-established component of my conceptual framework, in his study of educational motivation and self-efficacy, Wolters (2004) appeared to conceive of environmental (i.e., in this case, classroom) goal structures as being somewhat immutable, serving as a backdrop to which educators and researchers should attend but that cannot be readily modified. The trouble with this conceptualization, applying Gillborn's (2016) lens of white supremacy in education, is that it renders structural factors exempt from questioning or change; students' goals and motivations are interpreted within an educational system that has served, and continues to serve, to privilege a specific hegemonic approach to teaching and learning. To his credit, Wolters' study sample included several students (approximately 31\%) who identified themselves as an ethnicity other than White, but no mention was made of the role this did or did not play in contributing to the dependent variables of the study. I found this particularly troubling given what I felt to be the significance of motivation, goals, and self-efficacy in understanding the interaction between educational contexts and learning styles. By assuming that an educational environment simply is without questioning the degree to which it privileges a specific (White, western) learning style—or, alternately, is able to recognize, value, and sustain a multitude of cultural learning styles brought by studentsresearchers and educators reify fundamental system inequities while simultaneously rendering them invisible. Wolters' study, while intriguing, also serves as a caution to be 
thoughtful and mindful at all times throughout the planning and implementation of my study in order to avoid pitfalls such as these.

\section{Review of Methodological Literature}

Considering the variables and concepts I included in my exploration, the relationships I wished to assess, and the broad range of perspectives and voices I hoped to include, I employed a quantitative methodology (planned and implemented using a critical lens), with cross-sectional survey design serving as the particular study method. The foundation of literature and practice upon which I positioned this approach is in some ways rich and in others less robustly developed; the following paragraphs briefly locate my methodology within this body of literature, including both opportunities and limitations that I expected to encounter in the course of my study. Additional details regarding the specific activities constituting this study_-including sample, instrumentation, data collection and analysis procedures, and a reflection upon my positionality as a researcher-appear in Chapter 3 below.

Critical quantitative methodology. As I noted in the preceding pages, for my study of STEM education ecosystems as sources of cultural sustenance, I employed what I conceptualized as a critical quantitative methodological approach. This represented a bit of a break from tradition with regard to quantitative research in general and survey design studies in particular; across many of the most widely-used and frequently-cited texts regarding survey design research (e.g., Babbie, 1990, 2016; Fowler, 2014; Nardi, 2018), mentions of critical considerations and applications are scarce essentially to the point of nonexistence. However, while the pairing of quantitative methodologies and survey design methods with a critical paradigmatic orientation was far from the majority 
on either side of this Venn diagram, neither were they entirely nonexistent. In A Social Justice Approach to Survey Design and Analysis, Cornelius and Harrington (2014) argued, "One can use many approaches to design a survey using a social justice approach, and each is grounded by what the researcher thinks is the overall purpose of social justice research" (p. 22). This flexibility of methodological application is echoed by Guba and Lincoln (2005), who take the position that commensurability in conducting research may not exist between certain high-level paradigmatic worldviews, but methodologies in and of themselves are not by definition incompatible with different ontological, epistemological, and paradigmatic perspectives, critical and transformative frameworks included. Within the past decade, several researchers (e.g., Bowen \& Tillman, 2015; Doran, 2017; Gair, 2018; Han, 2010; Ngo, 2012; Ramos, 2012) have conducted quantitative survey research while applying a critical lens to their design and analysis. These studies and their particular relevance to my study are discussed in greater depth shortly; taken together, however, these and other researchers have offered thoughtful and valuable contributions to their respective areas of scholarship and have also set a strong precedent for the critical quantitative methodology I employed for my dissertation.

Cross-sectional survey design. Given the nature of the variables and relationships I wished to explore and the need to include the perspectives and contributions regarding agentic STEM education navigation from as large a sample as possible from across the population of Portland-area STEM learners between the ages of 14 and 18 years old, a survey design approach was well-suited to the goals and structure of my study. Of the two generally accepted forms of survey design researchlongitudinal and cross-sectional-I opted to employ a cross-sectional approach for my 
study, with data collected at a single point in time across a large sample of participants. The selection of a cross-sectional survey design introduced both opportunities and limitations, key among the latter being the increased difficulty in establishing causal relationships between variables. Whereas longitudinal studies are structured such that data collection occurs at multiple time points with a focus on change (descriptive and/or explanatory) over time, cross-sectional surveys focus on collecting data from a sample of participants a single time point with the purpose of generalizing descriptive findings and relationships between variables to a larger population. The inclusion of multiple time points in longitudinal survey research provides an inherent causal function to such studies; however, while limitations exist and must be acknowledged, techniques exist in both the design of cross-sectional studies (Babbie, 1990) and the analysis of crosssectional survey data (Visser et al., 2000) to facilitate a degree of causal inference. As Visser and colleagues (2000) noted:

cross-sectional surveys do offer the opportunity to assess relations between variables and differences between subgroups in a population. But although many scholars believe their value ends there, this is not the case. Cross-sectional data can be used to test causal hypotheses in a number of ways. (p. 225)

These and other scholars would doubtless agree that caution must be taken in making claims of causality on the basis of cross-sectional data, and the design of the study must be carefully constructed specifically to support such inferences. To the extent that I was able, I structured my study design, instrumentation, and data analysis approach in such a manner that some degree of causal examination could be included; however, because 
establishment of causal relationships was not finally the point of my study, any such findings should be considered "icing on the cake," as it were.

Established measures for conceptual framework. Of the four concepts that comprise the variables and outcomes I explored in this study (self-efficacy, STEM ecosystems, culturally sustaining pedagogy, and the six strands of science learning), it is worth noting that only one—-self-efficacy—wase currently well-represented in the literature with regard to established quantitative measures. From widely-utilized measures of general self-efficacy (Chen, Gully, \& Eden, 2001; Schwarzer \& Jerusalem, 1995; Sherer et al., 1982) to scales assessing self-efficacy in an academic setting (Zimmerman, Bandura, \& Martinez-Pons, 1992), the instrumentation and measurement of this construct has been well-document and robustly validated. Additionally, researchers have often employed certain "adjacent" conceptual constructs in studies of self-efficacy as stand-ins of sorts, foremost among these being the Motivational Strategies for Learning Questionnaire (Pintrich et al., 1993) and the Patterns of Adaptive Learning Scales (Midgley, Maehr, \& Urdan, 1993), each of which has been cited thousands of times across a multitude of research contexts. These established measures offered a wealth of options from which to choose in selecting questionnaire items for inclusion in my survey instrument; furthermore, had the particulars of my proposed study necessitated the preparation of bespoke self-efficacy measures, Bandura's (2006a) guide for the construction of self-efficacy scales offered an invaluable resource to facilitate such instrumentation.

In contrast to the wide range of established and validated measures available for the assessment of self-efficacy, as noted in the preceding pages, little if any literature 
existed to support the quantitative operationalization of (a) STEM ecosystems, (b)

culturally sustaining pedagogy, or (c) the six strands of science learning. With regard to the first, the framework developed by the STEM Funders Network (2016) offered a potential starting point for the development of instruments assessing learning experiences across areas of the Portland-area STEM education ecosystem, although I suspect further validation and refinement may be of value to strengthen the cultural, conceptual, and experiential resonance of this framework with learners. In terms of culturally sustaining pedagogy, there did not yet appear to be any quantitative research studies that existed among the body of published scholarship, with the exception of Blalock's (2013) dissertation study, which, as noted earlier, relied upon secondary analysis of existing survey data. However, the existence of scales assessing culturally responsive education, albeit from the perspective of teachers (e.g., Boon \& Lewthwaite, 2015; Hsiao, 2015; Rhodes, 2017; Siwatu, 2007; Whitaker \& Valtierra, 2018), offered a source of questionnaire items to be adapted for instruments intended for administration to learners rather than educators. Lastly, while the six strands had not yet been operationalized for the purposes of survey design research, their usage in my study as an organizing framework for the assessment of educational outcomes suggested that the language included in the $2009 \mathrm{NRC}$ report from which the six strands originated could serve as an appropriate source for what few questionnaire items were required for the purposes of instrumentation. Across all three of these concepts, this study provided an opportunity for further (and, in some cases, initial) development and validation of instruments that I and other researchers can continue to refine. 


\section{Summary}

In summation, it is clear that there has been no shortage of academic,

professional, and public attention dedicated to the persistent trends of inequitable STEM

field representation and to the possible reasons and ramifications attendant thereto. Past and contemporary scholars have explored and continue to explore the importance of individual motivations and attitudes in generating interest and engagement in these fields; likewise, I am far from the first to emphasize the importance of a critical perspective in assessing this subject. Recognizing the immensely complex and entangled nature of learning within an ecosystemic framework, I am grateful for the additional—and highly relevant-lens offered by complexity theory, and was eager to both draw upon and extend the sparse (but intriguing) literature regarding STEM learning ecosystems and the six strands of science learning. Taken together, the preceding pages have provided a theoretical and conceptual map of the landscape within which I conducted my study; in the following chapter, I build upon this map to outline my methodological approach, including instrumentation and data collection and analysis protocols, to address the research questions stated in Chapter 1. 
Chapter 3: Methods

In the preceding chapters, I have articulated a research problem I consider to be of great importance for the field of STEM education and situated this problem and my study within the body of extant literature generated by past and contemporary researchers and practitioners. Specifically, the problem I addressed through my research is that learners are not engaging in STEM or being supported in fostering STEM interest at equitable rates, and we as STEM educators do not sufficiently understand the individual and cultural motivations, needs, and agentic behavior of learners to provide culturally sustaining experiences and resources in support of individualized, learner-directed educational journeys. Building upon this foundation, the following chapter outlines in greater detail the methodological approach I employed for my study, including the methods used, the research method employed, the participants whose experiences and voices I hoped to foreground through my work, the procedures by which the study was undertaken, and my data collection and analysis activities. Additionally, while there appears to be a persistent perception within (and beyond) the field of educational research that the utilization of a quantitative methodology exempts the researcher from a careful examination of their own positionality (even at this university, quantitative dissertations are not required to explicitly address the role of the researcher [Portland State University, 2014]), I recognize the importance and significance of my unique presence inhabiting and guiding the proposed study, and this chapter therefore attempts to make visible and address these dynamics. 


\section{Study Overview}

As noted in the preceding sections, I employed a quantitative survey design study to explore the dynamics and cultural underpinnings of STEM learner attitudes, as well as how these attitudes and cultural backgrounds are sustained by various opportunities within the holistic Portland-area STEM education ecosystem and map to a range of STEM learning outcomes. The specific research questions I intended to address through this study led clearly to the selection of a quantitative methodology, as they are focused on the examination of relationships between variables; this focus paired with my intention to assess trends and dynamics across a broad population of learners further suggested survey design as an appropriate study method. Survey design, when properly constructed with appropriate attention to detail in the demarcation of sampling frames and the planning and implementation of sampling approaches, is well-suited to gathering data from a representative sample of a larger population (Fowler, 2014; Visser et al., 2000), one of the key goals of my study. Additionally, however, it was of paramount importance to me - and, I believe, to the integrity of my research and of my identity as a scholar - that I conduct this study and consider my findings through a critical lens. While survey design has only infrequently been utilized in this way, the work of other researchers who have conducted critically-oriented work through a quantitative survey design method (including but not limited to Doran, 2017; Ngo, 2012; and Ramos, 2012) provided guidance and assurance that a precedent existed for such scholarship.

\section{Participants}

For this survey, I specifically focused upon ninth- to twelfth-grade students recruited in a school district in Oregon's Willamette Valley. This age group was selected 
on the basis of alignment with OMSI visitorship and the body of scholarship that suggests that interest in pursuing a STEM career is strongly influenced by experiences prior to the age of 18 (Jiang et al., 2020; Kitchen et al., 2018; Sahin et al., 2017; Wang, 2013). Prior research has provided robust evidence that children as young as second grade and third grade (roughly 7-9 years old) are fully capable of possessing and demonstrating agency in their journeys of learning, both in STEM fields and in general (Adair, 2014; Varelas et al., 2012, 2015), offering further support for the selection of this age group as appropriate to the design and intent of the study.

Given the nature of my guiding research questions, the sampling frame I set for this study was restricted to ninth- to twelfth-grade students (the majority of whom fell between the ages of 14 and 18). While I hope that many if not all of the findings my participants and I have generated through this study will be at least conditionally generalizable to the broader audience of all students of similar age across the United States, such generalizability requires ongoing critical assessment throughout the course of data collection and analysis. I initially intended to employ a cluster sampling approach wherein I will would draw from a list of all public schools in the Portland metropolitan area that serve sixth, seventh, and eighth grade students $(N=74)$ based on the Oregon State School Directory, treating these schools as distinct clusters from which I would then randomly select a sample of schools $(n=12)$ for recruitment. However, due to the disruption caused by the COVID-19 pandemic, I was wholly unsuccessful in recruiting even a single school district for participation using this method, and opted instead to employ a purposive sampling technique whereby I sought permission to conduct my data collection activities at a single school district in Oregon's Willamette Valley. 
The selection of this particular district was made on the basis of its student demographics, which, being reflective of the demographics of the local community at large, includes a majority population of students who identify as racial and/or ethnic minorities. I recognized that the inclusion of a single school district (and a single high school within this district) had ramifications for the generalizability of my findings. However, in light of the conditions that precluded participation on the part of all districts I contacted during my initial recruitment attempts and the opportunity to learn from students of color in this particular district, in collaboration with my doctoral advisor, I determined that this course of action presented the greatest potential to provide meaningful and valuable learnings while foregrounding voices often minoritized in STEM fields. My decision to limit the sampling frame for this study to public schools rather than both public and private institutions was made in recognition of the fact that the demographics of private schools often demonstrate disproportionately high numbers of White students relative to public schools (Southern Education Foundation, 2016), limiting the opportunity to hear from learners of color in these spaces. This is by no means intended to diminish the importance of an exploration of the perspectives of students of color in private school contexts; indeed, such an exploration would likely be immensely valuable in illuminating the experiences, positive and negative, of these students, and I strongly recommend this as an avenue for future research by myself or other scholars.

\section{Procedures}

Upon the approval of this proposal, I was granted IRB approval for the inclusion of human participants in this study. Once I acquired IRB approval and identified the 
school district to be included in my study, I initiated conversations with the district superintendent to secure district-level buy-in and access before requesting permission from principals and teachers at the participating school to administer my survey directly to all currently enrolled students in participating teachers' STEM-focused virtual classrooms. Prior to delivery of survey links, I provided informed consent materials (Appendix A) to teachers and school administrators for review and inclusion in their communications. Once approval from school leadership was granted and informed consent procedures were conducted, I worked with teachers to ensure students had access to an electronic copy of my bilingual (Spanish/English) survey questionnaire. Participation by any given student was entirely voluntary, with no penalties for students who choose not to complete the questionnaire. All participating teachers were offered the opportunity to receive the raw, anonymized data for their participating classrooms, as well as up to eight tickets valid for general admission to OMSI, while all participating students received four tickets valid for OMSI general admission. I selected these incentives with the intention of striking a balance between a level of value indicative of my gratitude for the time and knowledge being shared and an avoidance of coercion due to an offer of items of excessive value. The nature of the incentive was also intended to address the disruption in classroom routine caused by my research activities — by offering complimentary access to informal STEM learning opportunities, albeit of a different nature and likely differing somewhat in specific content area focus, I hoped to offset the unavoidable decrease in classroom instructional time resulting from survey administration and completion. 


\section{Instruments and Measures}

The instrument I employed in the course of this study was a bilingual (Spanish and English) survey questionnaire combining items related to (a) self-efficacy, (b) culturally sustaining pedagogy, and (c) the six strands of science learning, assessed across (d) various areas of the STEM education ecosystem and including (e) relevant demographic information. Given the diverse sources and original purposes of established items, I modified wording as required to ensure that the language used was appropriate for the age group participating in this study, with the same lens applied to newly developed items. All scales and sub-scales were assessed for sufficient reliability using Cronbach's $\alpha$ based on a minimum reliability coefficient of .70, in line with generally accepted expectations of acceptability for internal consistency (Tavakol \& Dennick, 2011). Once I completed the initial construction of the survey instrument, the instrument was translated from English to Spanish by a bilingual/bicultural colleague. The Spanish version of my survey instruments is provided in Appendix B, while the English version of the survey instrument is available in Appendix C.

Self-efficacy. Self-efficacy questionnaire items were selected through a close review of established measures for this variable, including both general and academic self-efficacy as well as closely related motivational constructs often included in studies of self-efficacy (i.e., Chen et al., 2001; Midgley et al., 1993; Pintrich et al., 1993; Schwarzer \& Jerusalem, 1995; Sherer et al., 1982; Zimmerman et al., 1992). A full list of existing scales from which potential self-efficacy items were drawn is provided in Appendix D; these include both general self-efficacy scales and measures focused specifically upon learning and academic success. The eight specific items I selected for use in my survey 
instrument are adapted from Chen et al.'s (2001) general self-efficacy scale, with slight changes to wording in order to maximize accessibility and comprehensibility for youth participants.

Culturally sustaining pedagogy. Unlike self-efficacy, which is well-represented in the body of extant literature, culturally sustaining pedagogy has not previously been operationalized for the purposes of quantitative analysis. Fortunately, numerous researchers have explored the concept of culturally responsive pedagogy, which itself acted as the foundation upon which culturally sustaining pedagogy was proposed. Given the heritage of culturally sustaining pedagogy and its emergence from the framework of culturally responsive pedagogy, I compiled a comprehensive list of existing instruments (i.e., Boon \& Lewthwaite, 2015; Hsiao, 2015; Rhodes, 2017; Siwatu, 2007; Whitaker \& Valtierra, 2018) as a starting point in crafting my own questionnaire items, with the expectation that modifications and additions would be required to reflect both the conceptual nuances of culturally sustaining pedagogy and the different participant population-learners rather than educators - included in my study. (Appendix E provides a full list of existing scales addressing culturally responsive pedagogy that were be consulted as part of these efforts.) However, while I was grateful for this pool of established measures from which I could draw inspiration and that could serve as a basis for my own survey, I also recognized that significant and meaningful differences exist between culturally responsive and culturally sustaining pedagogy. Likewise, the survey instruments that had thus far been developed to assess culturally responsive pedagogy were largely directed at teachers and other educational practitioners as research participants, with few if any studies employing such instruments as a platform through 
which learners may share their experiences and perspectives. With this in mind, the 10 items I included in my survey instrument assessing culturally sustaining STEM experiences are adapted in part from existing measures of culturally responsive teacher readiness (particularly Hsiao, 2015; Rhodes, 2017; and Siwatu, 2007), supplemented with original items specifically related to the core concepts of culturally sustaining pedagogy. Across the 10 items comprising my newly developed culturally sustaining STEM learning scale (Appendix F), participants may share perceptions and experiences regarding foregrounding and recognition of legitimacy of cultural heritage and ways of knowing, the development of cultural pluralism, and accommodation of linguistic preferences. While this effort marks what I believe to be the first quantitative measure of culturally sustaining pedagogy, I hope to use this study as an opportunity for initial testing and validation of my proposed scale to assess the degree to which and ways in which the various items "hang" together conceptually overall (and by sub-construct if appropriate).

Other measures. Regarding the remaining components of the survey questionnaire, while the six strands of science learning had likewise not yet been operationalized for quantitative assessment, I used the core descriptions of the six strands (outlined in Table 1) as the primary source of inspiration in developing related questionnaire items, drawing additionally from the original 2009 NRC report as appropriate. Being cognizant of limited survey real estate, I included a single questionnaire item per strand, for a total of six questionnaire items. Variation of experience across the STEM ecosystem regions were assessed through replication of relevant questionnaire items, such that participants had the opportunity to report on their 
experiences and attitudes regarding self-efficacy, cultural sustenance, and alignment with the six strands for each of four ecosystem regions (school, science centers, other out-ofschool settings in their community, and home). Lastly, the demographic information I collected was limited to (a) gender, (b) race, (c) ethnicity, and (d) classroom, with the last of these being logged in the course of recruitment and survey collection rather than being asked directly of participants. For gender, the question was phrased in an open-ended manner to allow participants to state their gender identity in whatever way is most authentic to their lived experiences and sense of self. Race and ethnicity were constructed in a closed-ended fashion but allowed participants to select as many options as they wish, with an open-ended option available for those who preferred to articulate their identity in ways not captured by the pre-supplied choices. I preferred this construction for race and ethnicity out of a recognition that the usage of a purely openended approach would necessitate that I subsequently code and categorize responses to facilitate quantitative analysis, and I felt it is more respectful and appropriate to offer participants a structure at the outset (with the option for individualized expression) rather than rely entirely on post hoc categorization that would have introduced my own biases to a greater extent. All demographic questions were placed at the end of the survey instrument; while there is some evidence that placement of demographic questions at the beginning of a questionnaire increases the response rate for these questions without significantly affecting mean scores on other items (Teclaw, Price, \& Osatuke, 2012), the nature of the instrument and the potential relationship of other items to racial, ethnic, and gender identities made the minimization of risk of stereotype threat a vital consideration. 


\section{Role of the Researcher}

As I approached the planning and implementation of my study, I recognized the necessity of consciously positioning myself within my research and considering the ramifications of my experiences, my perspectives, my beliefs, and my biases in the framing of questions and interpretation of knowledge that would be shared with me by participants. My personal lived experience, as noted at the outset of this document, includes a homeschooled childhood during which I spent a good deal of time in science centers and museums; these experiences fundamentally shaped my perspective with regard to the importance and impactfulness of ISE institutions in sparking and fostering interest in STEM. In terms of ontological perspective, my worldview centers in large part upon the belief that each one of us has the ability, the obligation, and the privilege to contribute to the betterment of our world (and the lives of those within it) through our work and through our existence. I have learned that this outlook fits well with the transformative paradigm (Creswell, 2014; Mertens, 2010a, 2010b; Ravn, 2016) in terms of both ontology and epistemology, for as Mertens (2010b) suggested, "[transformative] researchers' understanding of the nature of reality (ontology) is influenced by their belief in the importance of respectfully addressing cultural diversity" (Mertens, 2010a, p. 12). Of equal and related importance, however, is my firm belief (which has both epistemological and methodological ramifications) that any attempt to explore and understand the co-created lived realities and experiences of individuals, groups, and cultures must begin not only with a focus on social justice but with an eye to the systemic structures and forces within which we are all perpetually and inextricably enmeshed. The immensely complex and dynamic interconnectivity between context and person are 
infused in every facet of the human experience, and can be seen mirrored in analogues throughout the natural world. This part of my ontological perspective leads me always to bear in mind the importance of remembering that "cell membranes taken from cells do not behave in the same way as they do in a cell. Likewise, a child taken from a classroom environment for assessment does not behave the same way as s/he does in a classroom" (Ricca, 2012, p. 36).

Epistemologically, this leads me to my belief that knowledge is interactional and jointly (and continually) co-constructed; I agree with Tolliver (2015) that "one knows oneself in relationship with others" (p. 62) and, more importantly, that "we change along with the world around us, and who we become is determined by how we react to change" (Bruce, 2002, p. 591). Interestingly, this perspective is in strong alignment with the epistemological outlook evident in the practices of some of the more cutting-edge and progressive museums. As Jeffers (2003) pointed out, "the alternative museum and its epistemology are concerned with the construction of knowledge within a group contextthat is, with how people construct a personal world from 'a labyrinth of potential connections' and seek to understand the relationship between their constructions and those of others" (p. 116). With this ontological and epistemological outlook present in my mind, I tend to shy away from the positivist notion that there exists any monolithic Truth that applies uniformly to all of humanity, and feel that even the search for little-t truths shared by individuals and groups is fraught with complexity and the dynamics of power and privilege. Given my preference, I would focus my energy primarily on quantitative inquiry; however, I am also fundamentally committed to the principles of the transformational paradigm and the centrality of participant voice and researcher 
positionality, as well as to the potential this paradigm holds for framing knowledge and inquiry so as to make visible the (often obscured) power structures that undergird and exacerbate social inequities (Mertens, 2010b). Importantly, in keeping with the tenets of a transformative epistemology (Mertens, 2010a), I feel that any methodological approach I employ must, to the greatest extent possible, include authentic and meaningful collaboration with participants and stakeholders in my research rather than treating participants simply as sources of data. With this in mind, whatever methodological approach may be called for in a given scenario, I believe that my role as a researcherand indeed, as a person who exists in the world —is "to consciously situate [my] work as a response to the inequities in society with a goal of enhancing social justice" (Mertens, 2010, p. 470).

Importantly, although I carry with me a multitude of ontological, epistemological, and methodological perspectives that at first glance may appear mutually incongruent, I do not experience these beliefs and preferences as conflicting with one another. Rather, I feel is it necessary to challenge the perception (which, based on the COE dissertation proposal rubric, is present even in the structures of our department) that quantitative research is somehow exempt from such considerations. To put this distinction another way and to clarify my positionality between, rather than within, the comfortable boundaries of paradigms, I will build on Phillips' (1990) description of the realist (i.e., objectivist) and relativist (i.e., subjectivist) approaches to reconciling different groups' conflicting understandings of their world and the phenomena therein. Whereas a realist, according to Phillips, believes that conflicting subjective realities may be legitimately experienced but that a single "true" reality exists (although it is not always possible to 
ascertain what it may be), a relativist can comfortably acknowledge the existence of multiple, and equally valid, apparently conflicting realities. So, then, does this cover the full range of paradigmatic interpretations of reality and our place in creating and experiencing it? I would argue that the answer is clearly "no." I believe there are stimuli, actions, objects, and so on that exist and can both act upon and be influenced by individual, groups, and societies - to suppose that each subjective reality will be entirely distinct and that the continuous co-construction of realities occurs without a common (or at least overlapping) set of referents runs contrary to my perspective on the navigation of life. However, and very importantly, the identification, mapping, or description of such "objective" (and I use the term loosely) stimuli is not finally the point of research on the human experience; it serves only as a red herring of sorts, distracting us from what is, for me, a crucial distinction between either of these worldviews and my personal paradigmatic perspective.

My position is this: Whether or not a "real" reality of shared stimuli exists, as I believe it does, it is in the exploration of our immensely complex and endlessly nuanced constructed and co-constructed experiences of and with these stimuli-and one anotherthat the charge of the social researcher (and, indeed, the human being) lies. I find myself resonating with Mertens (2010b) in her suggestion that "the transformative ontological assumption that there is one reality leads us to delve deeply into understanding factors that lead us to accept one version of reality over another" (p. 470). (Again, I will reiterate my belief that there is a foundational "real world" from and within which our subjectively-experienced realities emerge, but I would consider myself something of a 
practical agnostic with regard to the utility—or even the possibility—of attempting to assess this underlying world for the purposes of educational research.)

Where, then, does this leave me in terms of an ontological paradigm? Am I perhaps a pragmatist, believing as I do that the question of whether a "real" reality exists (and is able to be apprehended by any one of us who are simultaneously inhabiting our own somewhat idiosyncratic version thereof) is ancillary to my role as a researcher and a human being? At first glance this appears to be the case, for as Klingner and Boardman (2011) pointed out, "Researchers who adopt pragmatism are not necessarily interested in attempting to sort out epistemological/ontological issues. Rather, their interest lies in doing research that yields useful results (or results that work). They embrace various methodologies and perspectives" (p. 211). However, while this may be one piece of the complex picture I have found myself painting in attempting to visualize and articulate the ontological and epistemological paradigms from which I operate, it by no means captures all or even the most important parts of my worldview. To come at least somewhat closer to doing so, I will return once more to the foundational motivations and assumptions implicit in the transformative research paradigm. One of the most eloquent phrasings I have found of the fundamental distinction between a transformative and nontransformative is that "while the [hypothetico-deductive] method seeks to explain a present state of the world that the researcher has no desire to change, [transformative research] proceeds from an image of a desirable future" (Ackoff, 1974, as described in Ravn, 2016, p. 328). This perhaps more than anything else encapsulates my personal paradigm, particularly as pertaining to my identity as a researcher and as a leader in the field of education. Neutrality is not an option, and is in fact nothing but a fiction that has 
wrought more harm than good in social science research; it is incumbent upon each of us to strive through both scholarship and pedagogy to be agents of positive change and to contribute to a more equitable, healthy, and just society. These are the beliefs and perspectives that informed my positionality in my doctoral study — I have placed the highest degree of priority upon remaining mindful of my presence within my research and, with the support and thoughtful guidance of my peers and mentors, critically assessing potentially harmful influences of my background, perspectives, and beliefs in order to adjust my course when and if needed.

\section{Data Collection}

During the data collection process, I communicated with instructors and school administration to introduce myself, share information regarding the study, and provide survey links that could be distributed surveys to students who provide consent to participate. While my original intention was to distribute surveys in person using hard copies of the questionnaire, the COVID-19 pandemic necessitated a shift to entirely virtual education and research; this being the case, all surveys were distributed electronically as an optional Google Classroom assignment offered by participating teachers. Immediately following data collection, I contacted the students, teachers, and administrators involved to thank them once again for their collaboration and provide further information regarding the complementary OMSI general admission tickets offered as thanks for their participation.

\section{Data Analysis}

I conducted statistical analyses through the utilization of IBM SPSS Version 24, with a focus on exploring relationships between the independent and dependent (or 
outcome) variables outlined in the Instruments and Measures section above. Prior to analysis, all data were first downloaded in raw form and exported into a spreadsheet created using Microsoft Excel 2016 for cleaning and organization. Subsequent to completion of data cleaning, I began the analytic process by conducting descriptive statistical analyses of demographic variables and selected scales and subscales (e.g., culturally sustaining experiences, six strands of science learning outcomes). With regard to inferential statistical analyses, I planned to employ linear regression analyses, chisquare tests, and/or independent samples $t$ tests as appropriate to variable type, to assess the relationships between demographic characteristics and outcome variables; I also planned to employ chi-square tests to assess the distribution of categorical demographic variables relative to one another to identify any potentially problematic disproportionality within the participating sample. Additionally, I intended to conduct Pearson product moment correlations between outcome variable scales and, when these analyses indicated a statistically significant correlation between variables, planned to subsequently conduct linear regression analyses to assess potential causal relationships. However, I recognized that the smaller-than-anticipated sample size may result in data distributions that preclude parametric analyses; in the event that I deemed nonparametric tests necessary in light of such factors, I planned to implement Friedman tests as alternatives to ANOVAs, KruskalWallis $\mathrm{H}$ tests as alternatives to linear regressions, Mann-Whitney $\mathrm{U}$ tests as alternatives to independent samples $t$ tests, and Wilcoxon signed-rank tests as alternatives to the typical parametric post hoc tests associated with ANOVAs. (Please refer to Table 2 for a detailed outline of statistical tests proposed to assess each of the five research questions included in this study.) 
Table 2

Statistical Analyses, By Research Question

\begin{tabular}{ll}
\hline $\mathrm{RQ} \#$ & $\quad$ Statistical Analysis/es \\
\hline $\mathrm{RQ}_{1}$ & Friedman test \\
$\mathrm{RQ}_{2}$ & Kruskal-Wallis H test and Mann-Whitney U test \\
$\mathrm{RQ}_{3}$ & Friedman test \\
$\mathrm{RQ}_{4}$ & Kruskal-Wallis H test and/or Mann-Whitney U test \\
$\mathrm{RQ}_{5}$ & Chi-square analysis, Friedman test, and Wilcoxon signed-rank test
\end{tabular}

Through these analyses, I was excited to explore the research questions posed above and thereby to serve as a conduit for the knowledge, perspectives, and experiences generously shared by my participants. 


\section{Chapter 4: Results}

The preceding chapters have outlined the research problem on which this study focuses, grounded this problem in extant literature, and described the methodological approaches and procedures employed in the study. In the following pages, I present the results of the critical quantitative analyses used to explore and interpret the responses provided by the high school students who generously agreed to serve as participants and share their thoughts and experiences pertaining to STEM learning and engagement across the ecosystem. As noted above, data analysis was focused on addressing the following five overarching research questions:

$\mathrm{RQ}_{1}$ : In what ways do the feelings of self-efficacy expressed by Portland-area learners correspond with the various resource types in their local STEM education ecosystem?

$\mathrm{RQ}_{2}$ : In what ways do race, gender, and ethnicity impact the relationship between expressed self-efficacy and STEM ecosystem resource types among Portland-area learners?

$\mathrm{RQ}_{3}$ : To what extent do Portland-area learners report feelings of cultural sustenance when engaging with the various resource types in their local STEM education ecosystem?

$\mathrm{RQ}_{4}$ : In what ways do race, gender, and ethnicity impact the relationship between feelings of cultural sustenance and STEM ecosystem resource types among Portland-area learners? 
RQ5: In what ways do Portland-area learners indicate that their engagement in their various STEM ecosystem resources correspond to the learning outcomes proposed by the six strands of science learning?

Prior to the description of findings related to these questions, in the following pages, I begin with an overview of participant demographics, as well as the results of analyses pertaining to scale reliability and other variable-specific descriptive assessments. I selected statistical analyses for each of the five research questions based on the results of these preliminary assessments, as scale reliability, regularity or irregularity of data, and variable type (e.g., categorical or continuous) must all be borne in mind in order to ensure validity and meaningfulness of results. The five research questions driving this study are explored sequentially following the results of preliminary analyses; some interpretation is provided in this chapter, while further interpretation and a discussion of the ramifications of this study's findings overall are offered in Chapter 5.

\section{Participant Demographics}

All participants in this study were recruited from high school STEM classrooms in the Infinity School District; four instructors in this district consented to distribute the survey link to students as an optional assignment in their classes, resulting in a total of 19 virtual classrooms being invited to participate across two semesters. Classroom sizes ranged from approximately 25 to 32 students and participation by classroom ranged from zero to 20 (including both partial and complete responses), with a mean of 6.68 and a median of seven participants per classroom, for a total of 127 responses. Initial inspection of survey responses led to the removal of 34 responses that did not include at 
least one full screen of responses to items, leaving a total of 93 partial and complete survey responses included in the final dataset.

Among the 93 participants included in the following analyses, $47.3 \%(n=44)$ chose not to indicate their gender identity; of the 49 participants who did indicate their gender identity, 49\% $(n=24)$ identified as female, $46.9 \%(n=23)$ identified as male, and 4.1\% $(n=2)$ identified as non-binary. Slightly more participants $(51.6 \%, n=48)$ declined to provide their racial identity; of the 45 participants who responded to this question, $44.4 \%(n=20)$ identified as white, $2.2 \%(n=1)$ identified as American Indian/Alaskan Native, 2.2\% $(n=1)$ identified as Black or African-American, $11.1 \%(n=$ 5) identified as more than one race, and $40 \%(n=18)$ responded as "other," with one of these participant identifying as Eastern European and the remaining 17 identifying as Hispanic and/or Latin@. With regard to ethnicity, 39 participants indicated that they identified with one or more specific ethnic groups; of these participants, $84.6 \%(n=33)$ identified as Mexican, 2.6\% $(n=1)$ identified as Guatemalan, 5.1\% $(n=2)$ identified as American, 2.6\% $(n=1)$ identified as Vietnamese, 2.6\% $(n=1)$ identified as Russian, and $2.6 \%(n=1)$ identified as Hispanic with no further detail provided.

\section{Presentation and Analysis of Survey Data}

As described earlier, this research study employed a critical quantitative methodology relying upon a survey design method for collection of data. In the following pages, I present the results of survey data analysis, with analyses structured around the five research questions framing my exploration of learning across STEM ecosystems. I offer preliminary interpretation of findings for each of the five research 
questions in the respective subsection below; additional holistic interpretation of these findings is provided in the subsequent section.

Scale construction and reliability. Prior to conducting statistical analyses pertaining to the research questions stated above, I first combined items for self-efficacy (eight items per scale), culturally sustaining pedagogy (10 items per scale), and the six strands of science learning (six items per scale) and assessed the degree of internal reliability for each scale across the four ecosystem areas included in the survey. As illustrated in Table 3 below, with one exception, the results of these analyses indicated a degree of internal reliability that met or exceeded the 0.70 threshold of acceptability generally applied to Cronbach's $\alpha$ (Gliem \& Gliem, 2017), with the majority meeting the thresholds for "good" $(\geq .80)$ or "excellent" $(\geq .90)$ reliability levels.

Table 3

Initial Scale Composition and Reliability Assessments, Cronbach's a

Ecosystem Resource Area

\begin{tabular}{lccccc}
\cline { 2 - 6 } \multicolumn{1}{c}{ Scale } & \# of Items & Home & School & $\begin{array}{c}\text { Sci Ctrs/ } \\
\text { Museums }\end{array}$ & Other \\
\hline Self-Efficacy & 8 & .876 & .905 & .889 & .914 \\
CSP & 10 & .758 & .128 & .827 & .835 \\
Six Strands & 6 & .845 & .840 & .882 & .835
\end{tabular}

In reviewing the detailed results of these analyses, however, it became clear to me that the 10-item scale I had created to assess the experiences of learners' cultural sustenance was potentially flawed. Specifically, a review of the alpha levels with 
specific items deleted led me to the conclusion that the fifth item on the scale, "I need to turn my back on my cultural heritage if I want to be successful in learning about science," was contributing negatively to the alpha levels of all four instances of the CSP scale. Interestingly, this item was the only reverse-coded item in any of the scales (i.e., whereas a response of "Strongly Agree" on all other items translated to a higher level of the variable in question, a response of "Strongly Agree" on this item translated to a lower level of experienced cultural sustenance). The reverse-coding was accommodated during scale construction by recoding this variable into its inverse prior to inclusion in reliability assessments, but it is nonetheless possible that the grammatical construction of the item was confusing and/or non-intuitive for participants, particularly set alongside the other survey items that all employed a more standard direct-coding structure. In order to accommodate this issue, I removed this item from all four CSP scales and re-ran reliability assessments using the new nine-item construction. As shown in Table 4 below, the results of this analysis indicated a level of reliability for all scales that met or exceeded the 0.80 threshold of "good" $(\geq .80)$ reliability generally applied to Cronbach's $\alpha$ (Gliem \& Gliem, 2017), with some meeting the threshold for "excellent" ( $\geq .90)$ reliability levels. 
Table 4

Final Scale Composition and Reliability Assessments, Cronbach's a

Ecosystem Resource Area

\begin{tabular}{lccccc}
\cline { 2 - 6 } \multicolumn{1}{c}{ Scale } & \# of Items & Home & School & $\begin{array}{c}\text { Sci Ctrs/ } \\
\text { Museums }\end{array}$ & Other \\
\hline Self-Efficacy & 8 & .876 & .905 & .889 & .914 \\
CSP & 9 & .809 & .878 & .880 & .903 \\
Six Strands & 6 & .845 & .840 & .882 & .835
\end{tabular}

These scales, as outlined in Table 4, serve as the foundation for the statistical analyses I performed to explore the five research questions introduced earlier in this paper. Having said this, I urge continued examination and assessment of my newly created Culturally Sustaining STEM Experiences scale, with the goal of determining whether the reliability issues noted were an artifact of this particular implementation or if the revised nine-item scale is holistically more reliable across a range of research and learning settings.

Research question 1. In order to address the first research question regarding the relationship between expressed self-efficacy and various ecosystem resource types, I intended to assess the degree to which the central tendencies of self-efficacy scales were similar or different across ecosystem areas. In these analyses, ecosystem area served as the independent variable, while participant-reported levels of self-efficacy (a continuous variable comprised of multiple ordinal-level Likert-style items as outlined above) served as the dependent variable. Because more than two ecosystem area classifications existed, 
an independent samples $t$ test would not serve my purpose in this case; rather, the number, type, and configuration of these variables indicated a one-way analysis of variance (ANOVA) as an appropriate approach to exploring my questions regarding differences in mean self-efficacy levels across more than two ecosystem areas. This statistical test is designed to assess the difference in means on a continuous dependent variable between more than two (but no more than 26) groups included in a single independent categorical variable, provided three key assumptions are met (Field, 2018) specifically, that (a) all sources of variability have been accounted for, (b) the outcome is distributed normally (i.e., as a bell curve) for all groups, and (c) the degrees of variance exhibited by groups are homogeneous (i.e., not statistically different). In keeping with generally accepted protocol in the social sciences, I set the $\alpha$ level for all statistical tests described in the following pages at .05 .

To begin, I assessed the descriptive characteristics of all participants in the full sample included in my analysis; the resulting descriptive statistical information is provided in Table 5 below, corresponding histograms illustrating the distribution of selfefficacy scores across each ecosystem area are provided in Figure 4. (Note that lower scores equate to a higher proportion of responses of "Strongly Agree," indicating higher levels of self-efficacy.) Reviewing the descriptive information provided in Table 5, it is clear that some slight degree of difference exists in mean self-efficacy scores across groups (home $M=1.63$, school $M=1.51$, science center/museum $M=1.53$, other site $M$ $=1.65$ ), as well as in standard deviation (home $S D=.49$, school $S D=.51$, science center/museum $S D=.47$, other site $S D=.62$ ) and standard error (home $S E=.05$, school $S E=.07$, science center/museum $S E=.06$, other site $S E=.09)$. These means and 
standard deviations, when paired with a confidence interval of $95 \%$, indicate that the raw self-efficacy score for 95\% of participants in home settings would fall between .67 (1.63 $-[.49 \times 1.96])$ and $2.59(1.63+[.49 \times 1.96])$, while $95 \%$ of self-efficacy scores in school settings would fall between $.51(1.51-[.51 \times 1.96])$ and $2.51(1.51+[.51 \times 1.96]), 95 \%$ of self-efficacy scores in science center/museum settings would fall between .61 (1.53$[.47 \times 1.96])$ and $2.45(1.53+[.47 \times 1.96])$, and $95 \%$ of self-efficacy scores in other settings would fall between $.43(1.65-[.62 \times 1.96])$ and $2.87(1.65+[.62 \times 1.96])$. Likewise, the means and standard errors outlined in Table 1 indicate that the mean selfefficacy scores of $95 \%$ of random subsamples pulled from the same population would fall between $1.53(1.63-[.05 \times 1.96])$ and $1.73(1.63+[.05 \times 1.96])$ for home settings, between $1.37(1.51-[.07 \times 1.96])$ and $1.65(1.51+[.07 \times 1.96])$ for school settings, between $1.41(1.53-[.06 \times 1.96])$ and $1.65(1.53+[.06 \times 1.96])$ for science center/museum settings, and between $1.47(1.65-[.09 \times 1.96])$ and $1.83(1.65+[.09 \times$ 1.96]) for other settings.

Table 5

Self-Efficacy Descriptive Information by Ecosystem Resource Type

\begin{tabular}{lcccc}
\hline & $n$ & $M$ & $S D$ & $S E$ \\
\hline Home & 93 & 1.63 & .49 & .05 \\
School & 58 & 1.51 & .51 & .07 \\
Science Center/Museum & 53 & 1.53 & .47 & .06 \\
Other Sites & 51 & 1.65 & .62 & .09 \\
\hline
\end{tabular}




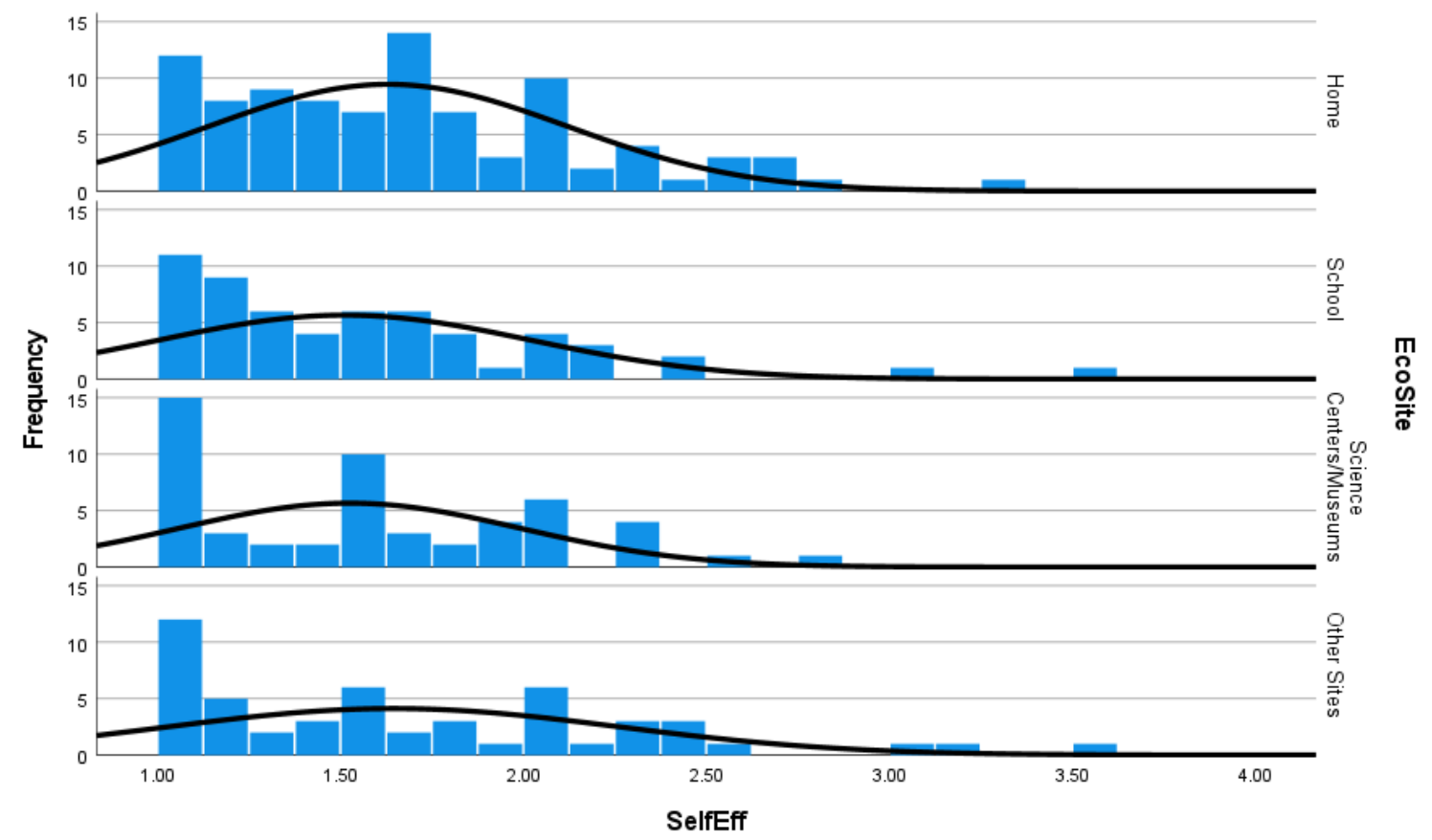

Figure 4. Overall distribution of self-efficacy scale scores across ecosystem resource type

Building from this descriptive foundation, I recognized that while the design of this study satisfies one assumption for the usage of ANOVA - that of accounting for extraneous sources of variability between groups - the lack of alignment between projected bell curves and actual distribution of data evident across all four groups in Figure 4 fails to satisfy a second assumption - that of normality. This being the case, I modified my statistical approach to instead include the Friedman test; in addition to serving as an accepted alternative to two-way ANOVAs (Bewick, Cheek, \& Ball, 2004), this test is generally considered an appropriate non-parametric alternative to one-way ANOVAs with repeated measures (Grice, Craig, \& Abramson, 2015). The four assumptions that must be met in order to employ the Friedman test (Field, 2018) are (a) that a single group of participants must be measured across three or more instances (in 
this case, each ecosystem assessment constituted an "instance"); (b) that the group is a random sample from the larger population; (c) that the dependent variable must be measured as ordinal or continuous (each self-efficacy scale in this case is constructed as a continuous variable); and (d) that samples do not need to be normally distributed. Unlike the assumptions underlying the usage of ANOVA, the distribution of my data meet these four assumptions for the Friedman test. The results of this test indicate that there was no statistically significant difference in expressed levels of self-efficacy across ecosystem resource type, $\chi^{2}(3)=1.925, p=.588$, suggesting that self-efficacy with regard to STEM learning and engagement is roughly consistent across ecosystem resource area among participants in this study.

Research question 2. Digging further into the details of participants' lived experiences with regard to self-efficacy across their STEM ecosystems requires that critical attention be paid to the influence and impact of gender and race on these experiences. Therefore, in order to address the second research question regarding any mediating or moderating effect of race and gender on expressed self-efficacy across various ecosystem resource types, I conducted additional analyses layering these identitybased variables into the statistical examination started above. In these analyses, race and gender served as the independent variables, while participant-reported levels of selfefficacy (a continuous variable comprised of multiple ordinal-level Likert-style items as outlined above) served as the dependent variable, with analyses conducted across each of the four ecosystem areas.

While my initial intention was to employ linear regression analyses or one-way ANOVAs as the analytic approach to assess the relationship between the variables stated 
in my second research question, the results of my analysis for the first research question made clear that the distribution of self-efficacy data are sufficiently irregular to preclude the usage of these tests. This being the case, and recognizing that both race and gender are categorical variables with three or more groups, but that in this situation it cannot be claimed that the same participants respond across multiple instances (as each ecosystem area will be analyzed separately), I instead employed the Kruskal-Wallis $\mathrm{H}$ test as a nonparametric alternative to the one-way ANOVA. As with the Friedman test outlined above, the Kruskal-Wallace test includes four assumptions (Field, 2018) that must be met in order to be employed, specifically (a) that the dependent variable must be measured as ordinal or continuous (each self-efficacy scale in this case is constructed as a continuous variable), (b) that the independent variable is categorical in nature and consists of two or more independent groups, (c) that there is no relationship between observations within or between groups, and (d) that samples do not need to be normally distributed (although non-normative data limit analysis to the comparison of mean ranks rather than comparison of medians. As outlined in the histograms in Figure 4, self-efficacy scale data are not normally distributed; hence, in the following analyses, I will only report on comparison of mean ranks among groups.

Before conducting Kruskal-Wallis $\mathrm{H}$ tests to assess the relationships between these variables, as with the preceding research question, I calculated descriptive information of self-efficacy scores by self-identified gender (Table 6), race (Table 7), and ethnicity (Table 8). (Again, note that lower scores equate to a higher proportion of responses of "Strongly Agree," indicating higher levels of self-efficacy.) The results of these descriptive analyses indicate small differences between participant groups, with 
generally smaller differences within participant groups across ecosystem sites. Standard deviations are modest and vary across groups; these deviations are most noticeable among groups with small numbers of participants, particularly those who reported their gender as non-binary, indicating notable variation across responses in these cases. On this note, these descriptive results illuminated the fact that distribution of responses was sparse across several categories of race. Since this distribution makes statistical analysis challenging if not impossible, I made the decision to consolidate into non-white identified and white-identified participants. While this decision is not without its limitations, foremost among these being the loss of individual identity as expressed by participants, the grouping remains true to the spirit of the research question — this being the exploration of whether and how different ecosystem resource types may or may not offer equitable opportunities for STEM self-efficacy among learners of color. Table 9 presents the descriptive information across racial categories under this operationalization; again, small differences in means are evident (although seemingly smaller than in earlier descriptive analyses), and it appears that standard deviations are slightly higher among white-identified participants than non-white identified participants. The reclassification of race into a binary variable also suggested the implementation of a Mann-Whitney $\mathrm{U}$ test rather than a Kruskal-Wallis $\mathrm{H}$ test to assess distribution of means across groups in this case. The assumptions and usages of the Mann-Whitney $U$ test are nearly identical to those of the Kruskal-Wallis $\mathrm{H}$ test, but whereas the latter can be employed with two or more categorical groups, the former is specialized for usage with independent categorical variables including only two groups. 
Table 6

Self-Efficacy Descriptive Information by Gender and Ecosystem Resource Type

\begin{tabular}{|c|c|c|c|c|c|}
\hline & & $n$ & $M$ & $S D$ & $S E$ \\
\hline \multirow[t]{4}{*}{ Female } & Home & 24 & 1.48 & .42 & .08 \\
\hline & School & 21 & 1.48 & .46 & .10 \\
\hline & Science Center/Museum & 22 & 1.49 & .46 & .10 \\
\hline & Other Sites & 22 & 1.58 & .56 & .12 \\
\hline \multirow[t]{4}{*}{ Male } & Home & 23 & 1.59 & .56 & .12 \\
\hline & School & 20 & 1.55 & .66 & .15 \\
\hline & Science Center/Museum & 19 & 1.55 & .53 & .12 \\
\hline & Other Sites & 20 & 1.62 & .70 & .16 \\
\hline \multirow[t]{4}{*}{ Non-Binary } & Home & 2 & 1.75 & 1.06 & .75 \\
\hline & School & 2 & 1.50 & .71 & .50 \\
\hline & Science Center/Museum & 2 & 1.88 & .62 & .44 \\
\hline & Other Sites & 2 & 3.13 & 1.50 & 1.06 \\
\hline
\end{tabular}


Table 7

Self-Efficacy Descriptive Information by Race and Ecosystem Resource Type

\begin{tabular}{|c|c|c|c|c|c|}
\hline & & $n$ & $M$ & $S D$ & $S E$ \\
\hline \multirow{4}{*}{$\begin{array}{l}\text { American Indian/Alaska } \\
\text { Native }\end{array}$} & Home & 1 & 2.00 & -- & -- \\
\hline & School & 1 & 2.13 & -- & -- \\
\hline & Science Center/Museum & 1 & 2.00 & -- & -- \\
\hline & Other Sites & 1 & 2.00 & -- & -- \\
\hline \multirow[t]{4}{*}{ Black/African-American } & Home & 1 & 2.29 & -- & -- \\
\hline & School & 1 & 1.25 & -- & -- \\
\hline & Science Center/Museum & 1 & 1.63 & -- & -- \\
\hline & Other Sites & 1 & 2.00 & -- & -- \\
\hline \multirow[t]{4}{*}{ White } & Home & 20 & 1.56 & .65 & .15 \\
\hline & School & 18 & 1.59 & .71 & .17 \\
\hline & Science Center/Museum & 19 & 1.53 & .51 & .12 \\
\hline & Other Sites & 18 & 1.74 & .80 & .19 \\
\hline \multirow[t]{4}{*}{ More than One Race } & Home & 5 & 1.28 & .20 & .09 \\
\hline & School & 4 & 1.25 & .35 & .18 \\
\hline & Science Center/Museum & 4 & 1.50 & .41 & .20 \\
\hline & Other Sites & 5 & 1.58 & .46 & .21 \\
\hline \multirow[t]{4}{*}{ Other } & Home & 18 & 1.51 & .40 & .75 \\
\hline & School & 16 & 1.39 & .29 & .50 \\
\hline & Science Center/Museum & 17 & 1.43 & .44 & .44 \\
\hline & Other Sites & 16 & 1.44 & .50 & 1.06 \\
\hline
\end{tabular}


Table 8

Self-Efficacy Descriptive Information by Ethnicity and Ecosystem Resource Type

\begin{tabular}{|c|c|c|c|c|c|}
\hline & & $n$ & $M$ & $S D$ & $S E$ \\
\hline \multirow[t]{4}{*}{ Latin@ } & Home & 33 & 1.61 & .47 & .08 \\
\hline & School & 30 & 1.57 & .50 & .09 \\
\hline & Science Center/Museum & 29 & 1.59 & .50 & .09 \\
\hline & Other Sites & 30 & 1.65 & .61 & .11 \\
\hline \multirow[t]{4}{*}{ Asian } & Home & 1 & 2.29 & -- & -- \\
\hline & School & 1 & 1.25 & -- & -- \\
\hline & Science Center/Museum & 1 & 1.63 & -- & -- \\
\hline & Other Sites & 1 & 2.00 & -- & -- \\
\hline \multirow[t]{4}{*}{ Other } & Home & 3 & 1.29 & .07 & .04 \\
\hline & School & 2 & 1.25 & .18 & .13 \\
\hline & Science Center/Museum & 2 & 1.25 & .18 & .13 \\
\hline & Other Sites & 2 & 1.19 & .09 & .06 \\
\hline \multirow[t]{4}{*}{ None/Not Selected } & Home & 51 & 1.68 & .51 & .07 \\
\hline & School & 21 & 1.52 & .57 & .13 \\
\hline & Science Center/Museum & 17 & 1.52 & .43 & .11 \\
\hline & Other Sites & 14 & 1.80 & .70 & .19 \\
\hline
\end{tabular}


Table 9: Self-Efficacy Descriptive Information by Race (Binary) and Ecosystem Resource Type

\begin{tabular}{llllll}
\hline & & $n$ & $M$ & $S D$ & $S E$ \\
\hline Non-White Identified & Home & 33 & 1.57 & .42 & .07 \\
& School & 30 & 1.46 & .40 & .07 \\
& Science Center/Museum & 29 & 1.53 & .45 & .08 \\
& Other Sites & 30 & 1.57 & .50 & .09 \\
White Identified & Home & 20 & 1.56 & .65 & .15 \\
& School & 18 & 1.59 & .71 & .17 \\
& Science Center/Museum & 19 & 1.53 & .51 & .12 \\
& Other Sites & 18 & 1.74 & .80 & .19 \\
\hline
\end{tabular}

Building upon these descriptive results, I conducted Kruskal-Wallis H tests to assess the distribution of mean self-efficacy ranks across gender and ethnicity, followed by a Mann-Whitney $U$ test assessing the distribution of mean self-efficacy ranks between non-white identified and white-identified participants. The results of the Kruskal-Wallis $\mathrm{H}$ test across the three categories of self-identified participant gender indicated no statistically significant differences in self-efficacy between any of the categories for the four ecosystem resource areas of home $(\mathrm{H}[2]=.207, p=.902)$, school $(\mathrm{H}[2]=.014, p$ $=.993)$, science centers and museums $(\mathrm{H}[2]=.140, p=.932)$, or other sites $(\mathrm{H}[2]=.066$, $p=.968)$. Similarly, the results of the Kruskal-Wallis $\mathrm{H}$ test across the four categories of self-identified participant ethnicity likewise indicated no statistically significant differences in self-efficacy between any of the categories for the four ecosystem resource areas of home $(\mathrm{H}[3]=4.094, p=.252)$, school $(\mathrm{H}[3]=1.257, p=.739)$, science centers 
and museums $(\mathrm{H}[3]=1.158, p=.763)$, or other sites $(\mathrm{H}[3]=1.869, p=.600)$. Lastly, the results of Mann-Whitney $\mathrm{U}$ tests indicated no significant difference in self-efficacy between non-white identified and white-identified participants in the four ecosystem resource areas of home (NWI $M d n=1.50$, WI $M d n=1.38, U=284.0, p=.395$ ), school (NWI $M d n=1.44$, WI $M d n=1.44, U=269.5, p=.991$ ), science centers and museums (NWI $M d n=1.50$, WI $M d n=1.50, U=261.0, p=.756$ ), or other sites (NWI $M d n=1.50$, WI $M d n=1.50, U=263.0, p=.738)$. In sum, these statistical analyses suggest that among participants in this study, race, gender, and ethnicity are not statistically significant factors in the experience of self-efficacy across STEM ecosystem resource areas.

Research question 3. In order to address the third research question regarding the relationship between feelings of cultural sustenance and various ecosystem resource types, I intended to assess the degree to which the central tendencies of cultural sustenance scales were similar or different across ecosystem areas. In these analyses, ecosystem area served as the independent variable, while participant-reported levels of perceived cultural sustenance (a continuous variable comprised of multiple ordinal-level Likert-style items as outlined above) served as the dependent variable. Because more than two ecosystem area classifications existed, an independent samples $t$ test would not serve my purpose in this case; rather, the number, type, and configuration of these variables indicated a one-way analysis of variance (ANOVA) as an appropriate approach to exploring my questions regarding differences in mean self-efficacy levels across more than two ecosystem areas. Of course, as noted in the preceding sections, the five assumptions of ANOVAs must necessarily be met in order to implement this test; if these 
assumptions were not met, I intended to employ the Friedman test similarly to its usage with regard to self-efficacy and ecosystem resource areas. In keeping with generally accepted protocol in the social sciences, as with the analyses described above, the $\alpha$ level for all statistical tests I describe in the following pages was set at .05.

To begin, I assessed the descriptive characteristics of all participants in the full sample included in my analysis; the resulting descriptive statistical information is provided in Table 10 below, with corresponding histograms illustrating the distribution of cultural sustenance scores across each ecosystem area are provided in Figure 5. (Note that lower scores equate to a higher proportion of responses of "Strongly Agree," indicating higher levels of perceived cultural sustenance.) Reviewing the descriptive information provided in Table 10, it is clear that some slight degree of difference exists in mean culturally sustaining learning experience scores across groups (home $M=1.91$, school $M=1.77$, science center/museum $M=1.70$, other site $M=1.92$ ), as well as in standard deviation (home $S D=.49$, school $S D=.55$, science center/museum $S D=.51$, other site $S D=.63$ ) and standard error (home $S E=.05$, school $S E=.07$, science center/museum $S E=.07$, other site $S E=.09)$. These means and standard deviations, when paired with a confidence interval of $95 \%$, indicate that the raw culturally sustaining learning experience score for $95 \%$ of participants in home settings would fall between .95 $(1.91-[.49 \times 1.96])$ and $2.87(1.91+[.49 \times 1.96])$, while $95 \%$ of self-efficacy scores in school settings would fall between $.69(1.77-[.55 \times 1.96])$ and $2.85(1.77+[.55 \times$ 1.96]), $95 \%$ of self-efficacy scores in science center/museum settings would fall between $.70(1.70-[.51 \times 1.96])$ and $2.70(1.70+[.51 \times 1.96])$, and $95 \%$ of self-efficacy 
scores in other settings would fall between $.69(1.92-[.63 \times 1.96])$ and $3.15(1.92+[.63$ $\times 1.96])$. Likewise, the means and standard errors outlined in Table 1 indicate that the mean culturally sustaining learning experience scores of $95 \%$ of random subsamples pulled from the same population would fall between $1.81(1.91-[.05 \times 1.96])$ and 2.01 $(1.91+[.05 \times 1.96])$ for home settings, between $1.63(1.77-[.07 \times 1.96])$ and $1.91(1.77$ $+[.07 \times 1.96])$ for school settings, between $1.56(1.70-[.07 \times 1.96])$ and $1.84(1.70+$ $[.07 \times 1.96])$ for science center/museum settings, and between $1.74(1.92-[.09 \times 1.96])$ and $2.10(1.92+[.09 \times 1.96])$ for other settings.

Table 10

Culturally Sustaining Learning Experience Descriptive Information by Ecosystem Resource Type

\begin{tabular}{lcccc}
\hline & $n$ & $M$ & $S D$ & $S E$ \\
\hline Home & 93 & 1.91 & .49 & .05 \\
School & 55 & 1.77 & .55 & .07 \\
Science Center/Museum & 53 & 1.70 & .51 & .07 \\
Other Sites & 51 & 1.92 & .63 & .09 \\
\hline
\end{tabular}




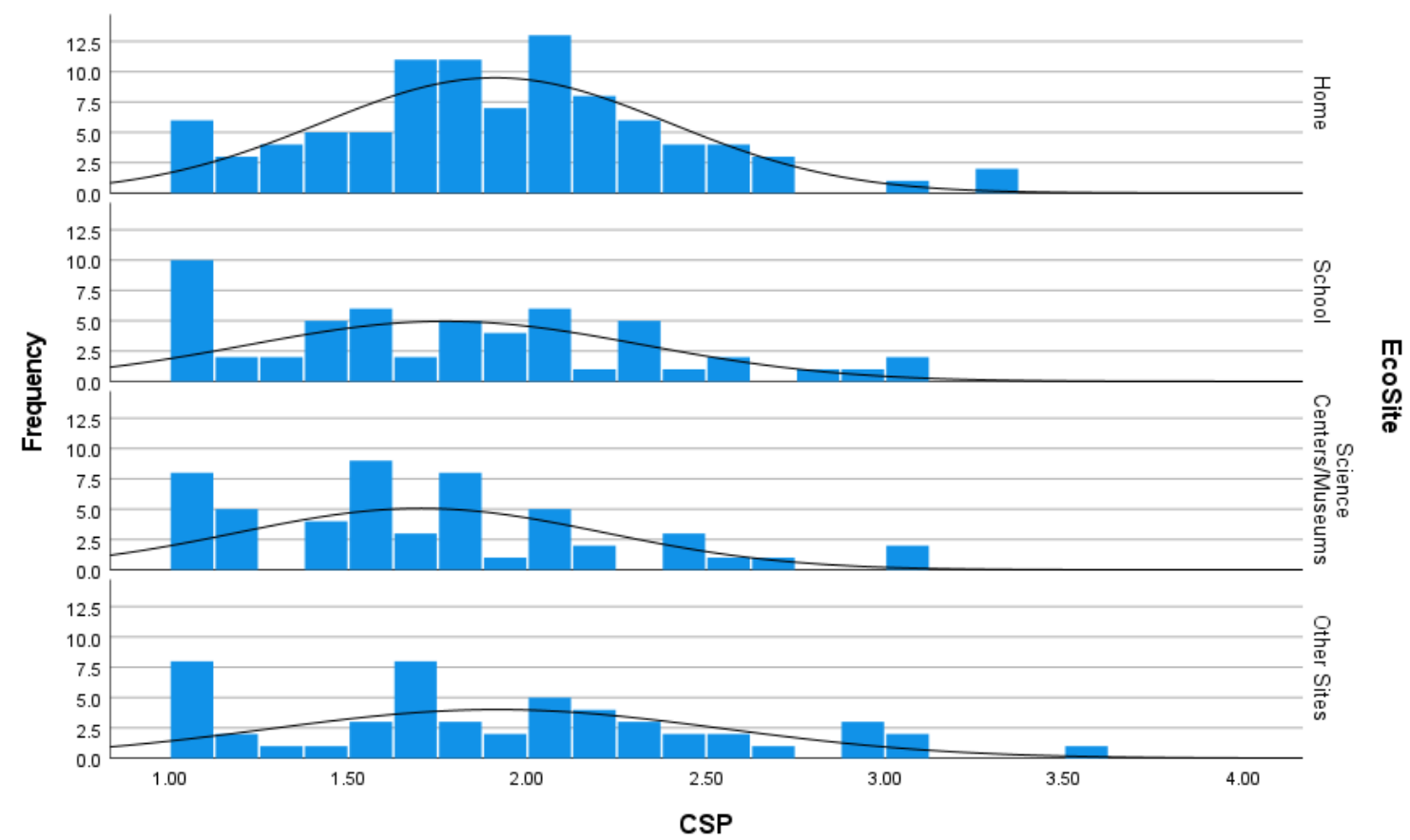

Figure 5. Overall distribution of culturally sustaining learning experience scale scores across ecosystem resource type

Building from this descriptive foundation, I recognized that as with my analyses of self-efficacy across ecosystem resource area, while the design of this study satisfies one assumption for the usage of ANOVA (Field, 2018) — that of accounting for extraneous sources of variability between groups - the lack of alignment between projected bell curves and actual distribution of data evident across all four groups in Figure 5 fails to satisfy a second assumption — that of normality. This being the case, I once again modified my statistical approach to instead include the Friedman test; as with my earlier self-efficacy analyses, unlike the assumptions underlying the usage of ANOVA, the distribution of my CSLE data meet these four assumptions for the Friedman test. The results of this test indicate that there was no statistically significant difference in expressed levels of cultural sustenance across ecosystem resource type, $\chi^{2}(3)=5.386$, 
$p=.146$, suggesting that the experience of cultural sustenance with regard to STEM learning and engagement is roughly consistent across ecosystem resource area among participants in this study.

Research question 4. In order to further explore the intricacies of participants' lived experiences with regard to feelings of cultural sustenance across their STEM ecosystems, I intended to incorporate an analysis of gender and race into my initial examination of these experiences. Therefore, in order to address the fourth research question regarding any mediating or moderating effect of race and gender on culturally sustaining learning experiences across various ecosystem resource types, I conducted additional analyses layering these identity-based variables into the statistical examination started above. In these analyses, race and genders served as the independent variables, while participant-reported levels of cultural sustenance in STEM learning (a continuous variable comprised of multiple ordinal-level Likert-style items as outlined above) served as the dependent variable, with analyses conducted across each of the four ecosystem areas.

While my initial intention, as with my earlier analyses of self-efficacy, was to employ linear regression analyses or one-way ANOVAs as the analytic approach to assess the relationship between the variables stated in my second research question, the results of my analysis for the third research question indicated that the distribution of culturally sustaining learning experience data, as with self-efficacy data earlier, are sufficiently irregular to preclude the usage of these tests. This being the case, and recognizing that both race and gender are categorical variables with three or more groups but that in this situation it cannot be claimed that the same participants respond across 
multiple instances (as each ecosystem area will be analyzed separately), I again employed the Kruskal-Wallis $\mathrm{H}$ test as a nonparametric alternative to the ANOVA. The four assumptions of the Kruskal-Wallis test described in the section regarding RQ2 are equally applicable, and equally well-fulfilled, in these analyses; as outlined in the histograms in Figure 5, CSLE scale data are not normally distributed, meaning the following analyses will only report on comparison of mean ranks among groups.

Before conducting Kruskal-Wallis $\mathrm{H}$ tests to assess the relationships between these variables, as with the preceding research question, I calculated descriptive information of CSLE scores by self-identified gender (Table 11), race (Table 12), and ethnicity (Table 13). (Again, note that lower scores equate to a higher proportion of responses of "Strongly Agree," indicating higher levels of perceived cultural sustenance.) The results of these descriptive analyses indicate small differences between participant groups, with generally smaller differences within participant groups across ecosystem sites. Standard deviations are modest and vary across groups; these deviations are most noticeable among groups with small numbers of participants, particularly those who reported their gender as non-binary, indicating notable variation across responses in these cases. On this note, these descriptive results illuminated the fact that distribution of responses was sparse across several categories of race. Since this distribution makes statistical analysis challenging if not impossible, I made the decision to consolidate into non-white identified and white-identified participants. While this decision is not without its limitations, foremost among these being the loss of individual identity as expressed by participants, the grouping remains true to the spirit of the research question - this being the exploration of whether and how different ecosystem resource types may or may not 
offer equitable opportunities for STEM self-efficacy among learners of color. Table 9 presents the descriptive information across racial categories under this operationalization; again, small differences in means are evident (although seemingly smaller than in earlier descriptive analyses), and it appears that standard deviations are slightly higher among white-identified participants than non-white identified participants. The reclassification of race into a binary variable also suggested the implementation of a Mann-Whitney U test rather than a Kruskal-Wallis $\mathrm{H}$ test to assess distribution of means across groups in this case. The assumptions and usages of the Mann-Whitney $U$ test are nearly identical to those of the Kruskal-Wallis H test, but whereas the latter can be employed with two or more categorical groups, the former is specialized for usage with independent categorical variables including only two groups. 
Table 11

Culturally Sustaining Learning Experience Descriptive Information by Gender and Ecosystem Resource Type

\begin{tabular}{|c|c|c|c|c|c|}
\hline & & $n$ & $M$ & $S D$ & $S E$ \\
\hline \multirow[t]{4}{*}{ Female } & Home & 24 & 1.96 & .60 & .12 \\
\hline & School & 21 & 1.88 & .61 & .13 \\
\hline & Science Center/Museum & 22 & 1.73 & .51 & .11 \\
\hline & Other Sites & 22 & 2.04 & .65 & .14 \\
\hline \multirow[t]{4}{*}{ Male } & Home & 23 & 1.79 & .53 & .11 \\
\hline & School & 19 & 1.60 & .56 & .13 \\
\hline & Science Center/Museum & 19 & 1.59 & .53 & .12 \\
\hline & Other Sites & 20 & 1.79 & .65 & .15 \\
\hline \multirow[t]{4}{*}{ Non-Binary } & Home & 2 & 1.61 & .86 & .61 \\
\hline & School & 2 & 1.89 & 1.26 & .89 \\
\hline & Science Center/Museum & 2 & 1.72 & 1.02 & .72 \\
\hline & Other Sites & 2 & 1.56 & .79 & .56 \\
\hline
\end{tabular}


Table 12

Culturally Sustaining Learning Experience Descriptive Information by Race and Ecosystem Resource Type

\begin{tabular}{|c|c|c|c|c|c|}
\hline & & $n$ & $M$ & $S D$ & $S E$ \\
\hline \multirow{4}{*}{$\begin{array}{l}\text { American Indian/Alaska } \\
\text { Native }\end{array}$} & Home & 1 & 1.89 & -- & -- \\
\hline & School & 1 & 2.22 & -- & -- \\
\hline & Science Center/Museum & 1 & 2.13 & -- & -- \\
\hline & Other Sites & 1 & 2.11 & -- & -- \\
\hline \multirow[t]{4}{*}{ Black/African-American } & Home & 1 & 2.33 & -- & -- \\
\hline & School & 1 & 1.78 & -- & -- \\
\hline & Science Center/Museum & 1 & 2.50 & -- & -- \\
\hline & Other Sites & 1 & 2.89 & -- & -- \\
\hline \multirow[t]{4}{*}{ White } & Home & 20 & 1.91 & .61 & .14 \\
\hline & School & 17 & 1.78 & .59 & .14 \\
\hline & Science Center/Museum & 19 & 1.68 & .46 & .11 \\
\hline & Other Sites & 18 & 1.87 & .57 & .13 \\
\hline \multirow[t]{4}{*}{ More than One Race } & Home & 5 & 1.58 & .49 & .22 \\
\hline & School & 4 & 1.42 & .46 & .23 \\
\hline & Science Center/Museum & 4 & 1.58 & .39 & .19 \\
\hline & Other Sites & 5 & 1.80 & .56 & .25 \\
\hline \multirow[t]{4}{*}{ Other } & Home & 18 & 1.81 & .56 & .13 \\
\hline & School & 16 & 1.69 & .57 & .14 \\
\hline & Science Center/Museum & 17 & 1.58 & .45 & .11 \\
\hline & Other Sites & 16 & 1.80 & .79 & .20 \\
\hline
\end{tabular}


Table 13

Culturally Sustaining Learning Experience Descriptive Information by Ethnicity and Ecosystem Resource Type

\begin{tabular}{|c|c|c|c|c|c|}
\hline & & $n$ & $M$ & $S D$ & $S E$ \\
\hline \multirow[t]{4}{*}{ Latin@ } & Home & 33 & 1.91 & .47 & .08 \\
\hline & School & 29 & 1.81 & .54 & .10 \\
\hline & Science Center/Museum & 29 & 1.78 & .53 & .10 \\
\hline & Other Sites & 30 & 1.96 & .66 & .12 \\
\hline \multirow[t]{4}{*}{ Asian } & Home & 1 & 2.33 & -- & -- \\
\hline & School & 1 & 1.78 & -- & -- \\
\hline & Science Center/Museum & 1 & 2.50 & -- & -- \\
\hline & Other Sites & 1 & 2.89 & -- & -- \\
\hline \multirow[t]{4}{*}{ Other } & Home & 3 & 1.85 & .74 & .43 \\
\hline & School & 2 & 1.33 & .16 & .11 \\
\hline & Science Center/Museum & 2 & 1.39 & .24 & .17 \\
\hline & Other Sites & 2 & 1.33 & .47 & .33 \\
\hline \multirow[t]{4}{*}{ None/Not Selected } & Home & 51 & 1.94 & .46 & .06 \\
\hline & School & 19 & 1.84 & .55 & .13 \\
\hline & Science Center/Museum & 16 & 1.63 & .40 & .10 \\
\hline & Other Sites & 14 & 1.96 & .52 & .14 \\
\hline
\end{tabular}


Table 14

Culturally Sustaining Learning Experience Descriptive Information by Race (Binary) and Ecosystem Resource Type

\begin{tabular}{llllll}
\hline \multirow{2}{*}{ Non-White Identified } & $n$ & $M$ & $S D$ & $S E$ \\
& Home & 33 & 1.84 & .52 & .09 \\
& School & 29 & 1.73 & .58 & .11 \\
& Science Center/Museum & 29 & 1.68 & .51 & .09 \\
Other Sites & 31 & 1.91 & .67 & .12 \\
& Home & 20 & 1.91 & .61 & .14 \\
& School & 17 & 1.78 & .59 & .14 \\
& Science Center/Museum & 19 & 1.68 & .46 & .11 \\
& Other Sites & 18 & 1.87 & .57 & .13 \\
\hline
\end{tabular}

Building upon these descriptive results, I conducted Kruskal-Wallis $\mathrm{H}$ tests to assess the distribution of mean culturally sustaining learning experience ranks across gender and ethnicity, followed by a Mann-Whitney $U$ test assessing the distribution of mean culturally sustaining learning experience ranks between non-white identified and white-identified participants. The results of the Kruskal-Wallis H test across the three categories of self-identified participant gender indicated no statistically significant differences in reported cultural sustenance between any of the categories for the four ecosystem resource areas of home $(\mathrm{H}[2]=1.16, p=.559)$, school $(\mathrm{H}[2]=2.54, p=.281)$, science centers and museums $(\mathrm{H}[2]=1.07, p=.587)$, or other sites $(\mathrm{H}[2]=1.81, p$ $=.404)$. Similarly, the results of the Kruskal-Wallis $\mathrm{H}$ test across the four categories of self-identified participant ethnicity likewise indicated no statistically significant 
differences in reported cultural sustenance between any of the categories for the four ecosystem resource areas of home $(\mathrm{H}[3]=1.42, p=.701)$, school $(\mathrm{H}[3]=2.07, p=.559)$, science centers and museums $(\mathrm{H}[3]=3.97, p=.265)$, or other sites $(\mathrm{H}[3]=4.16, p$ $=.245)$. Lastly, the results of Mann-Whitney U tests indicated no significant difference in reported cultural sustenance between non-white identified and white-identified participants in the four ecosystem resource areas of home (NWI $M d n=1.78$, WI $M d n=$ 1.78, $U=317.5, p=.818$ ), school (NWI $M d n=1.67$, WI $M d n=1.56, U=237.5, p$ $=.837$ ), science centers and museums (NWI $M d n=1.67$, WI $M d n=1.56, U=270.5, p$ $=.916)$, or other sites (NWI $M d n=1.78$, WI $M d n=1.83, U=275.5, p=.942)$. In sum, these statistical analyses suggest that as with self-efficacy before, among participants in this study, race, gender, and ethnicity are not statistically significant factors in the experience of cultural sustenance across STEM ecosystem resource areas. It is interesting to note, however, that while none of these analyses indicated a statistically significant relationship between variables, the $p$ values were generally lower than those observed for self-efficacy. Further exploration is necessary to continue assessing possible connections between experiences of cultural sustenance and the ways in which learners navigate their STEM ecosystems.

Research question 5. Lastly, as a conclusion to my analyses, I wished to assess the degree to which different STEM learning ecosystem areas may align, or not, with the outcomes articulated in the six strands of science learning, as well as with the six strands taken together. In order to conduct the former assessments, I intended to employ chisquare analyses with ecosystem resource area (a categorical variable) as one crosstabulation component and the six individual six strands survey items (each 
constructed ordinally) as the second component of each crosstabulation. For the latter assessment, meanwhile, I intended to conduct a one-way ANOVA with the same categorical variable of ecosystem resource area as the independent variable and the Six strands scale (a continuous variable comprised of multiple ordinal-level Likert-style items, in keeping with my self-efficacy and CSLE scales) serving as the dependent variable. My earlier analyses, however, made clear the importance of first conducting descriptive analyses in order to ensure the distribution of responses satisfy the assumptions underlying the parametric ANOVA (Field, 2018); in the event that these assumptions were not met, a Friedman test served as my nonparametric contingency plan.

Bearing this in mind, as a first step in this final round of analyses, I assessed the descriptive characteristics of all participants in the full sample with regard to the six strands; the resulting descriptive statistical information is provided in Table 15 below, corresponding histograms illustrating the distribution of composite six strands scale scores across each ecosystem area are provided in Figure 6. (Note that lower scores equate to a higher proportion of responses of "Strongly Agree," indicating higher levels of alignment with the six strands overall.) Reviewing the descriptive information provided in Table 15, it is clear that some slight degree of difference exists in mean six strands scale scores across groups (home $M=1.89$, school $M=1.65$, science center/museum $M=1.57$, other site $M=1.78$ ), as well as in standard deviation (home $S D$ $=.56$, school $S D=.55$, science center/museum $S D=.58$, other site $S D=.60)$ and standard error (home $S E=.06$, school $S E=.07$, science center/museum $S E=.08$, other site $S E=.08$ ). These means and standard deviations, when paired with a confidence interval of 95\%, indicate that the raw self-efficacy score for $95 \%$ of participants in home 
settings would fall between $.79(1.89-[.56 \times 1.96])$ and $2.99(1.89+[.56 \times 1.96])$, while $95 \%$ of self-efficacy scores in school settings would fall between $.57(1.65-[.55 \times 1.96])$ and $2.73(1.65+[.55 \times 1.96]), 95 \%$ of self-efficacy scores in science center/museum settings would fall between $.43(1.57-[.58 \times 1.96])$ and $2.71(1.57+[.58 \times 1.96])$, and $95 \%$ of self-efficacy scores in other settings would fall between $.60(1.78-[.60 \times 1.96])$ and $2.96(1.78+[.60 \times 1.96])$. Likewise, the means and standard errors outlined in Table 1 indicate that the mean self-efficacy scores of $95 \%$ of random subsamples pulled from the same population would fall between $1.77(1.89-[.06 \times 1.96])$ and $2.01(1.89+$ $[.06 \times 1.96])$ for home settings, between $1.51(1.65-[.07 \times 1.96])$ and $1.79(1.65+[.07 \times$ 1.96]) for school settings, between $1.41(1.57-[.08 \times 1.96])$ and $1.73(1.57+[.08 \times$ 1.96]) for science center/museum settings, and between $1.62(1.78-[.08 \times 1.96])$ and $1.94(1.78+[.08 \times 1.96])$ for other settings.

Table 15

Six Strands of Science Learning Scale Information by Ecosystem Resource Type

\begin{tabular}{lcccc}
\hline & $n$ & $M$ & $S D$ & $S E$ \\
\hline Home & 93 & 1.89 & .56 & .06 \\
School & 55 & 1.65 & .55 & .07 \\
Science Center/Museum & 52 & 1.57 & .58 & .08 \\
Other Sites & 51 & 1.78 & .60 & .08 \\
\hline
\end{tabular}




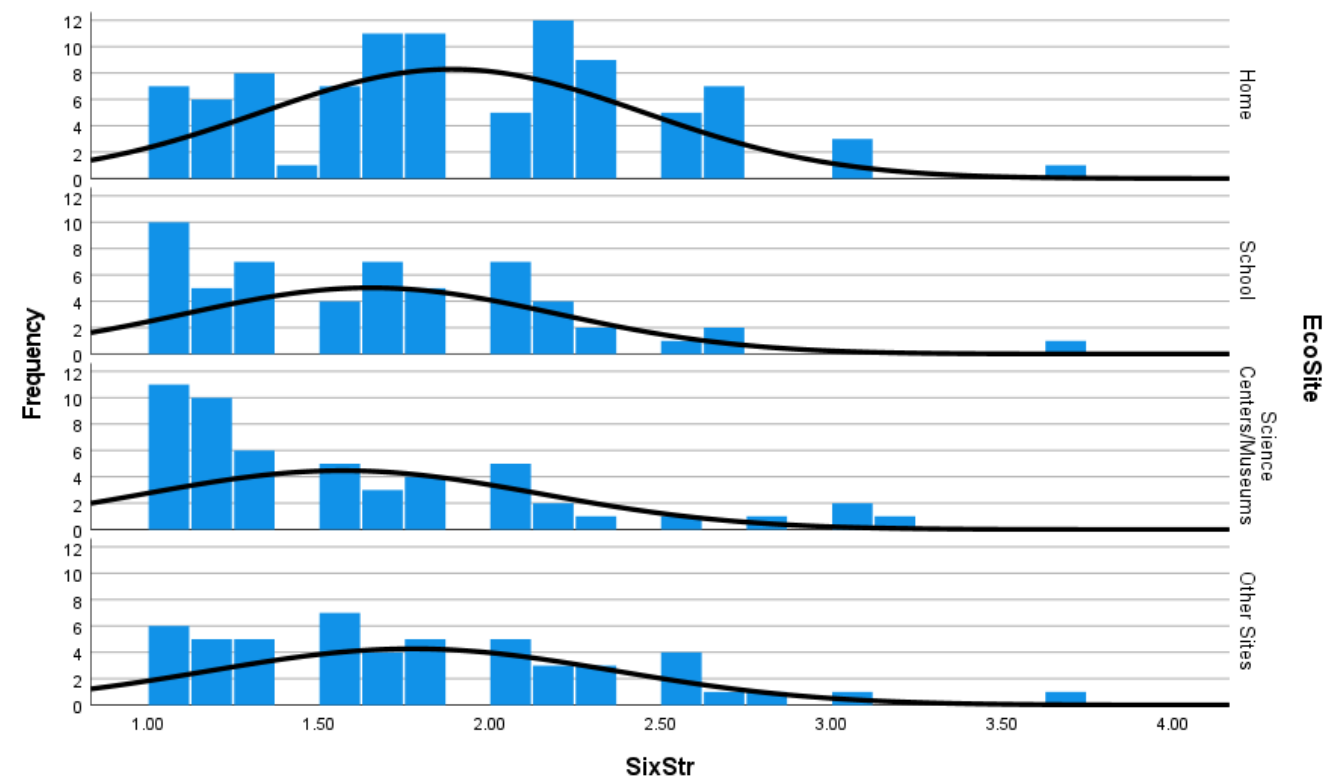

Figure 6. Overall distribution of six strands of science learning scale scores across ecosystem resource type

Building from this descriptive foundation, I recognized that as with my earlier analyses of self-efficacy and culturally sustaining learning experiences across ecosystem resource area, while the design of this study satisfies one assumption for the usage of ANOVA (Field, 2018) — that of accounting for extraneous sources of variability between groups - the lack of alignment between projected bell curves and actual distribution of data evident across all four groups in Figure 6 fails to satisfy the assumption of normality. This being the case, I once again modified my statistical approach to instead include the Friedman test; as with my earlier self-efficacy analyses, unlike the assumptions underlying the usage of ANOVA, the distribution of my six strands scale data meet these four assumptions for the Friedman test. The results of this test indicate a strongly statistically significant difference in expressed levels of alignment with the six strands of science learning across ecosystem resource type, $\chi^{2}(3)=19.73, p<.001$, suggesting that 
the experience of outcomes associated with the six strands varied significantly based on ecosystem resource area among participants in this study.

This significance warrants further examination to better understand precisely where the differences in mean ranks fell among the four ecosystem resource areas, and because the Friedman test does not directly permit the examination of such category-bycategory differences, I recognized that I would need to conduct a series of either Wilcoxon signed-rank tests or paired-samples sign tests assessing each of the six pairings between the four ecosystem categories (Morgan, 2002). The three underlying assumptions of the Wilcoxon signed-rank test (Field, 2018) are (a) that the dependent variable should be either ordinal or continuous in nature, (b) that the independent variable should be comprised of two categorical "matched pairs," and (c) that the distribution of differences between groups must be symmetrical. The first two of these three assumptions were met by the nature of the variables in question, but further examination is necessary to confirm that the third assumption is likewise satisfied. In order to assess the symmetry of differences between groups, I computed six new variables, one for each of the six pairings between the four ecosystem categories, and each subtracting one six strands ecosystem area scale from another (e.g., "SixStrHomeSchoolDiff" subtracted the value for the six strands school-site scale from the value for the six strands home-site scale). Figure 7 presents the Q-Q plots illustrating the distribution of differences across the six pairings; while some outliers exist in each case, the distributions of these data indicate that across all six comparisons, the assumption of symmetry is largely met, satisfying the third and final assumption required for the usage of Wilcoxon signed-rank tests. 
A MULTIPLICITY OF JOURNEYS
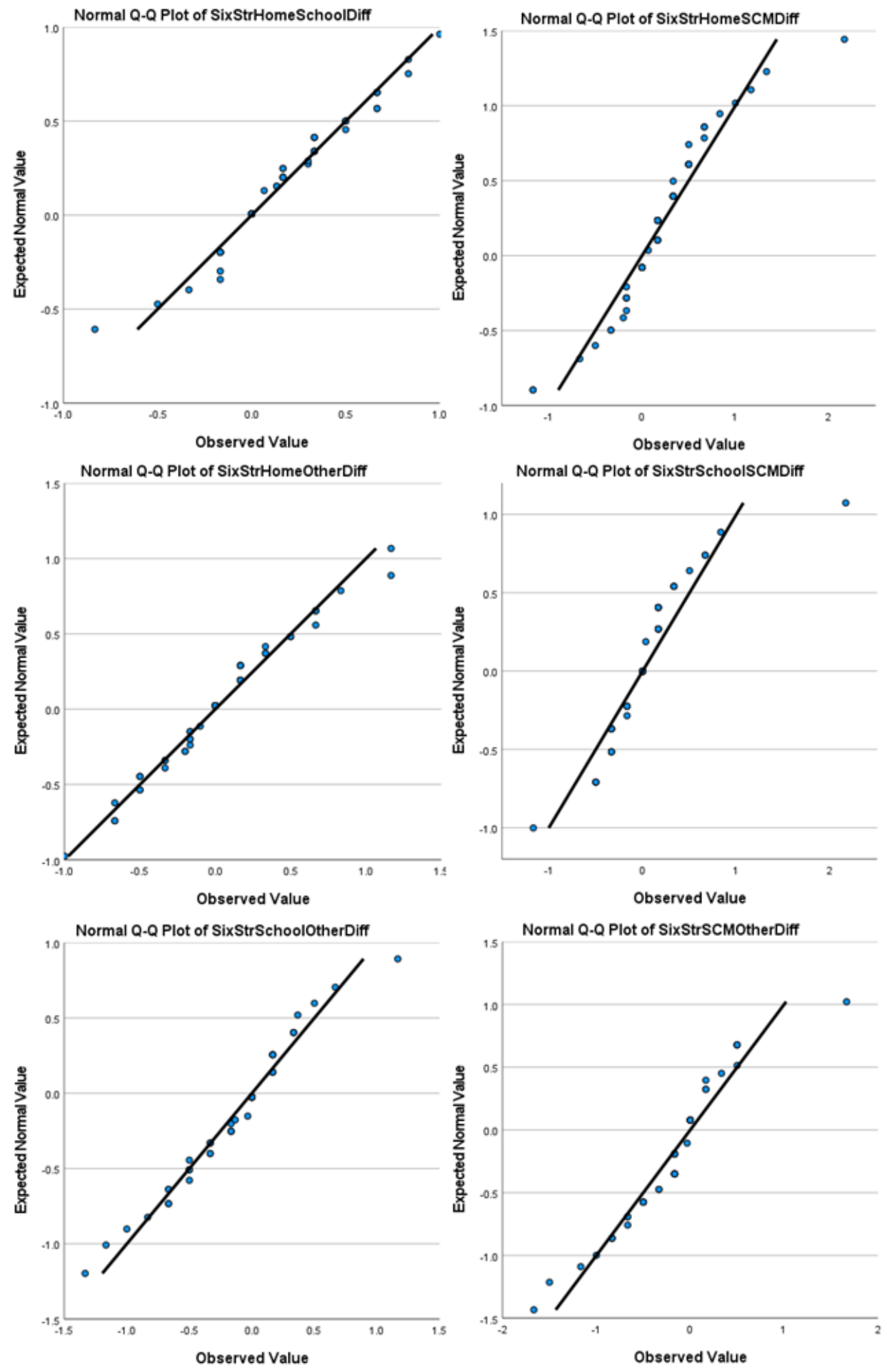

Figure 7. Q-Q Plots of Symmetry of Six Strands Scale Data Distribution, Differences Between Ecosystem Resource Area 
With the three underlying assumptions met, I proceeded to conduct Wilcoxon signed-rank tests assessing the specific differences in mean ranks between each of the four ecosystem resource areas. Before reporting the results of these assessments, it is important to note that per Field (2018), a Bonferroni adjustment is required to ensure that statistical significance is not inaccurately calculated through a Type I error. In order to calculate the appropriate Bonferroni adjustment, I divided the standard significance level of .05 by the number of tests I performed (in this case, six), for a revised significance threshold of .008 , meaning that any tests yielding a $p$ value of greater than .008 will not be considered statistically significant. Bearing in mind this adjusted significance value, the results of the six Wilcoxon signed-rank tests indicated that statistically significant differences do not exist between mean six strands scale ranks in home settings (mean rank $=21.36$ ) and other site settings (mean rank $=19.55), \mathrm{Z}=-.521, p=.603$; between ranks in school settings (mean rank $=15.61$ ) and science center/museum settings (mean rank $=18.67), \mathrm{Z}=-.009, p=.993$; or between school settings (mean rank $=15.96)$ and other site settings (mean rank $=21.56), \mathrm{Z}=-2.142, p=.032$. However, these analyses also indicated that statistically significant differences $d o$ exist between mean six strands scale ranks in home settings (mean rank $=22.55$ ) and school settings (mean rank $=$ 16.20), $\mathrm{Z}=-3.499, p<.001$; between ranks in home settings (mean rank $=23.58)$ and science center/museum settings (mean rank $=21.42$ ), $\mathrm{Z}=-2.959, p=.003$; and between science center/museum settings (mean rank $=18.78)$ and other site settings $($ mean rank = 19.07), $\mathrm{Z}=-2.775, p=.006$.

These results are intriguing, but only tell part of the story with regard to how, precisely, the six strands of science learning align with different resource areas in 
learners' STEM ecosystems. Therefore, in addition to the composite six strands scale described above, my analyses for my final research question require an examination of each of the six strands survey questions taken individually. Table 16 presents the descriptive information for each of these six survey items across the four ecosystem resource areas; because my planned analyses of these variables will treat all the survey items involved as ordinal rather than continuous, this table includes frequencies only, rather than central tendencies. 
Table 16

Six Strands of Science Learning Survey Item Information by Ecosystem Resource Type

Survey Response Count (\%)

\begin{tabular}{|c|c|c|c|c|c|c|}
\hline & & \multirow{2}{*}{\multicolumn{3}{|c|}{ Strongly }} & \multirow{2}{*}{\multicolumn{2}{|c|}{ Strongly }} \\
\hline & & & & & & \\
\hline & & $n$ & Agree & Agree & Disagree & \\
\hline \multirow{2}{*}{$\begin{array}{l}\text { Strand 1: } \\
\text { Developing } \\
\text { Interest in }\end{array}$} & Home & 93 & $43(46.2)$ & $43(46.2)$ & $7(7.5)$ & $0(0.0)$ \\
\hline & School & 55 & $37(67.3)$ & $16(29.1)$ & $1(1.8)$ & $1(1.8)$ \\
\hline \multirow[t]{2}{*}{ Science } & Science Center/Museum & 52 & $34(65.4)$ & $13(25.0)$ & $4(7.7)$ & $1(1.9)$ \\
\hline & Other Sites & 51 & $27(52.9)$ & $18(35.3)$ & $5(9.8)$ & $1(2.0)$ \\
\hline \multirow{2}{*}{$\begin{array}{l}\text { Strand 2: } \\
\text { Understanding } \\
\text { Science }\end{array}$} & Home & 92 & $32(34.8)$ & $45(48.9)$ & $14(15.2)$ & $1(1.1)$ \\
\hline & School & 55 & $21(38.2)$ & $27(49.1)$ & $5(9.1)$ & $2(3.6)$ \\
\hline \multirow[t]{2}{*}{ Knowledge } & Science Center/Museum & 52 & $29(55.8)$ & $16(30.8)$ & $7(13.5)$ & $0(0.0)$ \\
\hline & Other Sites & 51 & $25(49.0)$ & $19(37.3)$ & $6(11.8)$ & $1(2.0)$ \\
\hline \multirow{4}{*}{$\begin{array}{l}\text { Strand 3: } \\
\text { Engaging in } \\
\text { Scientific } \\
\text { Reasoning }\end{array}$} & Home & 92 & $23(25.0)$ & $51(55.4)$ & $14(15.2)$ & $4(4.3)$ \\
\hline & School & 55 & $25(45.5)$ & $26(47.3)$ & $3(5.5)$ & $1(1.8)$ \\
\hline & Science Center/Museum & 52 & $24(46.2)$ & $22(42.3)$ & $5(9.6)$ & $1(1.9)$ \\
\hline & Other Sites & 51 & $18(35.3)$ & $22(43.1)$ & $9(17.6)$ & $2(3.9)$ \\
\hline \multirow{4}{*}{$\begin{array}{l}\text { Strand 4: } \\
\text { Reflecting on } \\
\text { Science as a Way } \\
\text { of Knowing }\end{array}$} & Home & 93 & $37(39.8)$ & $46(49.5)$ & $8(8.6)$ & $2(2.2)$ \\
\hline & School & 54 & $29(53.7)$ & $20(37.0)$ & $4(7.4)$ & $1(1.9)$ \\
\hline & Science Center/Museum & 52 & $35(67.3)$ & $12(23.1)$ & $4(7.7)$ & $1(1.9)$ \\
\hline & Other Sites & 50 & $26(52.0)$ & $21(42.0)$ & $2(4.0)$ & $1(2.0)$ \\
\hline Strand 5: & Home & 93 & $19(20.4)$ & $36(38.7)$ & $29(31.2)$ & $9(9.7)$ \\
\hline $\begin{array}{l}\text { Engaging in } \\
\text { Scientific }\end{array}$ & School & 53 & $20(37.7)$ & $21(39.6)$ & $10(18.9)$ & $2(3.8)$ \\
\hline Practices & Science Center/Museum & 52 & $26(50.0)$ & $20(37.7)$ & $6(11.5)$ & $0(0.0)$ \\
\hline
\end{tabular}




\begin{tabular}{|c|c|c|c|c|c|c|}
\hline & Other Sites & 51 & $10(19.6)$ & $24(47.1)$ & 9 (17.6) & $8(15.7)$ \\
\hline Strand 6: & Home & 93 & $28(30.1)$ & 49 (52.7) & $14(15.1)$ & $2(2.2)$ \\
\hline $\begin{array}{l}\text { Identifying with } \\
\text { the Scientific }\end{array}$ & School & 55 & $26(47.3)$ & $22(40.0)$ & $6(10.9)$ & $1(1.8)$ \\
\hline \multirow[t]{2}{*}{ Enterprise } & Science Center/Museum & 52 & 27 (51.9) & $17(32.7)$ & $7(13.5)$ & $1(1.9)$ \\
\hline & Other Sites & 51 & $27(52.9)$ & $18(35.3)$ & $4(7.8)$ & $1(3.9)$ \\
\hline
\end{tabular}

At first glance, the distribution of responses across the six strands appears to suggest some variation by ecosystem resource type, but deeper analysis is required to determine whether statistically significant relationships exist between ecosystem area and science learning strand. In order to explore this possibility, I conducted chi-square analyses crosstabulating ecosystem resource area with each of the six strands. The results of these analysis indicate that no statistically significant relationships exist between ecosystem resource type and Strand $1, \chi^{2}(9, N=251)=13.531, p=.140$; between ecosystem resource type and Strand $2, \chi^{2}(9, N=250)=11.119, p=.268$; between ecosystem resource type and Strand $3, \chi^{2}(9, N=250)=12.841, p=.170$; between ecosystem resource type and Strand $4, \chi^{2}(9, N=249)=12.097, p=.208$; or between ecosystem resource type and Strand $6, \chi^{2}(9, N=251)=12.313, p=.196$. A strongly statistically significant relationship does exist, however, between ecosystem resource type and Strand 5 "Engaging in scientific practices," $\chi^{2}(9, n=249)=30.055, p<.001$. The detailed distribution of responses in this crosstabulation paint a picture of responses that are generally more positive and less negative than would be proportionally expected with regard to science center/museum settings and generally less positive and more negative than would be proportionally expected with regard to home settings. 
A MULTIPLICITY OF JOURNEYS

\section{Overall Interpretation of Findings}

Taken together, these findings begin to paint a picture of learners' engagement with different resources across their STEM learning ecosystems. While statistical significance was generally scarce with the exception of differences across ecosystem area in outcomes associated with the six strands of science learning, it is still possible to note some potential trends that warrant further exploration. In particular, while no significant differences were observed in experiences of cultural sustenance either across ecosystem resource area or across demographic category, a visual inspection of the mean scores for culturally sustaining learning experiences appears to suggest a generally greater degree of variability for this concept than for self-efficacy across both ecosystem area and demographic groups. Bearing in mind the tenets of quantitative methodologies, it is important to emphasize the absence of statistical significance associated with any such variations among groups. However, from a critical perspective, it is our responsibility as researchers to attend carefully and thoughtfully to the stories being told by the data entrusted to us by participants, and while this particular study does not provide evidence of significant relationships between these variables, the underlying visual inequities in the distribution of means may speak to phenomena that can be more deeply understood through further study.

I spiritedly reject the notion that only statistically significant results are deserving of consideration or distribution to broad audiences; this notion, and the attendant phenomenon of publication bias (i.e., the tendency of academic journals to disproportionately favor the publication of manuscripts featuring statistically significant analysis results), are well-documented in the literature (e.g., Ferguson \& Heene, 2012; 
Gerber \& Malhotra, 2008; Lin \& Chu, 2018) and have far-reaching negative ramifications for the robustness of learning made available to the field. I likewise, however, recognize within myself the desire to seek meaning where I believe it should be found - for I confess that I feel, both on the basis of my thorough examination of the literature and on a deeply personal level based on my own experiences, that experiences of cultural sustenance should, and perhaps must, vary across engagement with different elements of one's STEM learning ecosystem. Recognizing this internal bias, I am also mindful that I must be open to the possibility that my suppositions are incorrect; certainly they are not borne out at a statistically significant level in the present study, so while I do posit that the findings of this research project suggest potentially valuable avenues for continued scrutiny, I wish to avoid overstepping the bounds of ethical and scrupulous interpretation.

As noted above, the one point in my analyses where statistical significance was met was in regard to the distribution of mean scores associated with the Six strands of science learning. In reviewing these findings, I was strongly reminded of the complexity of educational and learning settings; as noted by Cochran-Smith et al. (2014), educational processes and environments constitute highly complex systems, with multidimensional and dynamic relationships and connections between the various resources, locations, and individuals therein. This being the case, it is particularly interesting to note that while (a) significant differences in composite six strands mean ranks were evident between science center/museum settings and both home settings and other sites and (b) the National Research Council originally proposed Strands 1 and 6 as being particularly relevant to and aligned with the nature of informal STEM learning 
environments (NRC, 2009), neither Strand 1 nor Strand 6 demonstrated any noteworthy differences in positivity or negativity of response distribution. These findings strongly suggest that further exploration is merited to assess the extent to which educators, administrators, and researchers working in informal STEM learning settings may need to reconsider their assumptions regarding the types of learning outcomes best supported by the environments in which we work and teach.

\section{Limitations of Study}

As is the case with all research efforts, several limitations existed that I have borne in mind when considering the interpretation of these findings and that I encourage readers to likewise incorporate into their critical lenses. First, it is inarguably the case that the size of the participating sample was not optimal, particularly for a quantitative study. This limitation was largely an artifact of the specific moment in history during which I conducted my study, as the COVID-19 pandemic resulted in massive disruption to formal education systems across the country and around the world; whatever the cause, however, the end result is that fewer schools (and, resultantly, fewer students) were able to find bandwidth to participate in a doctoral project initiated by someone unaffiliated with their particular districts. Reflecting on this limitation, I considered implementing bootstrapping where possible throughout my analyses in order to artificially extrapolate what the results may have looked like had larger sample sizes been possible, but I ultimately reached the conclusion that a thoughtful, thorough, and honest examination of the data as they are is a truer representation of a critical quantitative methodology. This being the case, while these findings do, I believe, offer intriguing pathways for future 
exploration, taken on their own, they are not as robust as I might originally have hoped when planning my study in a pre-COVID world.

A second and related limitation of this study is the inclusion of a single school rather than the multiple randomly selected schools originally planned in my proposed sampling approach. Again, this limitation is a result of the disruption caused by the COVID-19 pandemic throughout the field of formal education, with the vast majority of districts scrambling to adapt to a rapidly shifting teaching and learning environment and seeking to support students and families in crisis throughout these unprecedented times. Administrators and educators rightly dedicated every moment of time and ounce of energy to quickly identify and implement creative solutions, offer meaningful learning opportunities, and provide broad-spectrum support and resources for communities of learners who were suddenly thrust into a model of engagement unlike any they had experienced before. While these dynamics unavoidably caused limitations in participation across districts, I am inexpressibly grateful to the school district included in my study for offering me the opportunity to connect with students, to the teachers of participating STEM classrooms for extending the invitation to their students across multiple semesters, and to the students themselves for taking the time to share their thoughts and experiences in spite of all that was happening in their worlds. Fortunately, my sample of students did include a substantial number of learners who identified as students of color as well as a fairly equitable distribution across gender identity, offering some measure of insight into STEM learning experiences across demographic groups. Nevertheless, this limitation unavoidably bounded the extent of generalization made possible through this exploratory research study, and I again encourage further 
examination of the concepts and preliminary findings of this study in order to build upon the humble foundation I have offered herein.

Lastly, by my employment of a critical quantitative methodology, I recognize that I cannot offer the equally important body of findings that might be gleaned through the use of qualitative or mixed methods. This limitation is simply an expected and unavoidable result of the methodology I chose to employ, and is not intended to imply that any research project can or should seek to yield a truly comprehensive understanding of such a complex and multifaceted experience as the process of learning across the STEM ecosystem. Rather, I note this limitation in order to highlight the need for continued research efforts to advance the field of education broadly, formal and informal alike, through qualitative as well as quantitative inquiry. The stories of our lived experiences can — and should—be meaningfully told through numbers and words alike, and this particular quantitative study is intended to be only one thread of a much larger tapestry woven along with those who have come before and those who will follow. In the following chapter, I will further explore these limitations and the opportunities they suggest with regard to future research, alongside the overall ramifications of this exploratory research study. 


\section{Chapter 5: Discussion}

In the preceding four chapters, I have articulated a persistent problem of practice encountered by educators and leaders in the STEM fields, particularly but not exclusively those working in informal learning environments. Specifically, I have observed that learners are not engaging in STEM or being supported in fostering STEM interest at equitable rates, and we as STEM educators do not sufficiently understand the individual and cultural motivations, needs, and agentic behavior of learners. Furthermore, we do not fully grasp how learners engage with resources and providers within their holistic STEM ecosystems to avail themselves of culturally sustaining experiences and resources as they chart their own educational journeys. In grappling with this problem of practice and through a grounding in the scholarship that has been generated by past and contemporary researchers in the fields of formal and informal education, STEM, race and gender studies, and many others that bound and enriched my understanding of the dynamics at play, I was led to explore the following five research questions:

$\mathrm{RQ}_{1}$ : In what ways do the feelings of self-efficacy expressed by Portland-area learners correspond with the various resource types in their local STEM education ecosystem?

$\mathrm{RQ}_{2}:$ In what ways do race, gender, and ethnicity impact the relationship between expressed self-efficacy and STEM ecosystem resource types among Portland-area learners?

$\mathrm{RQ}_{3}$ : To what extent do Portland-area learners report feelings of cultural sustenance when engaging with the various resource types in their local STEM education ecosystem? 
$\mathrm{RQ}_{4}$ : In what ways do race, gender, and ethnicity impact the relationship between feelings of cultural sustenance and STEM ecosystem resource types among Portland-area learners?

$\mathrm{RQ}_{5}$ : In what ways do Portland-area learners indicate that their engagement in their various STEM ecosystem resources correspond to the learning outcomes proposed by the Six strands of science learning?

I was called to confront and critically examine this problem as a result of my personal positionality and lived experiences. From my childhood as a homeschooled learner whose STEM identity was strongly shaped by visits to science centers and museums in the Pacific Northwest, to my present role as an educational researcher at a large Portland-area ISE institution, the opportunities provided by spaces like these to foster STEM interest and self-efficacy have long been near and dear to my heart. My love and respect for ISE environments has, however, also begun to be troubled by the incontrovertible fact that even within such spaces, planned and staffed by dedicated and caring educators and administrators, we have in many cases struggled to engage in meaningful, culturally sustaining ways with learners from communities (particularly racial and ethnic communities) historically minoritized in STEM fields. Science centers and museums, as with the majority of institutions comprising the museum field, are steeped in heritages rife with colonialism, white supremacy, and inequitable access for and representation of women and communities of color (Dawson, 2014, 2018; Domínguez, Weffer, \& Embrick, 2020; Tolia-Kelly, 2016). If we are ever to redress these historical and contemporary harms and begin writing a new and more equitable future for the field of informal STEM education, it is fundamentally important that we 
begin by centering the voices, experiences, and agency of learners and that we seek to understand and honor the motivations that guide them through their exploration of the ecosystems in which they live.

This final chapter serves as a culmination and synthesis of the primary and secondary research I have undertaken in the course of this study, but far more than this, it should be recognized for what it truly is: a celebration of the experiences of those learners who chose to share their perspectives through their survey responses, and an invitation for us all, as educators, researchers, and leaders, to serve with humility and seek to elevate the voices that have far, far too often been systematically marginalized and excluded. The preceding chapters have grounded this exploration in my personal lived experiences as well as the body of literature that has emerged through the scholarship and contributions of the many educators, researchers, and community members who have paved the way with their own curiosity and drive to understand the perspectives and motivations of learners. I have offered critical interpretations of the results of my statistical analyses with a specific goal of addressing the five research questions guiding this study; in these last pages, I wish to turn my attention to a holistic consideration of my findings, particularly considering their implications for educational practice and future research.

\section{Synthesis of Findings}

First and foremost, I feel it is both necessary and intriguing to reiterate the fact that few of the analyses I undertook resulted in statistically significant results. What is often overlooked, as a result of the bias of academic journals to publish research findings that demonstrate statistical significance (Ferguson \& Heene, 2012; Lin \& Chu, 2018), is 
that findings of statistical non-significance can themselves hold as much conceptual significance as those at or below the established $p$ value threshold. In this case, while further exploration is inarguably merited to both validate and extend the findings from this initial foray into quantitative assessment of ecosystemic STEM learning, the absence of statistically significant variances in self-efficacy or feelings of cultural sustenance across ecosystem areas, even when taking into account race, ethnicity, and gender, is counterintuitive and therefore intriguing. I would be remiss in failing to confess that these findings may be due at least in part to the fact that my methodology, and particularly the instrumentation I employed to operationalize my variables of study, are largely newly developed and essentially untested beyond the bounds of the current exploration. I will address this point and other methodological considerations in greater detail below, but do wish to highlight that even bearing this in mind, in keeping with the tenets of complexity theory, there may be additional dynamics at play that require consideration beyond those that are immediately evident on the surface of our assessments of gendered and raced experiences of learning (Cochran-Smith et al., 2014).

One possibility that comes immediately to mind when I consider the outcomes of this study is that an operationalization of ecosystemic learning in the modern era may benefit from some incorporation of digital or e-learning environments. Over the past two decades, digital learning systems have grown from occasional anomalies to become a ubiquitous component of the educational landscape (Arguel, Lockyer, Lipp, Lodge, \& Kennedy, 2016; Grand-Clement, Devaux, Belanger, \& Manville, 2017; Mladenova, Kalmukov, \& Valova, 2020). While in-person learning experiences have largely remained "the norm," digital experiences have by their very nature contributed to the 
blurring of ecosystemic lines, as learners can now, for example, engage in an "einternship program" and gain industry experience from their school classrooms (Crusio, Rubino, \& Delprato, 2017), or perhaps visit a "digital museum" without ever leaving their homes (Gran, Vestberg, Booth, \& Ogundipe, 2019; Grincheva, 2014). It is also worth noting that I conducted all data collection for my study during the peak of the COVID-19 pandemic, a moment in history during which the majority of formal educational institutions (including K-12 schools) were operating using a model of comprehensive or partial distance learning (Alqahtani \& Rajkhan, 2020; Mladenova et al., 2020; Van Nuland, Hall, \& Langley, 2020); in other words, digital learning had, seemingly overnight, become the norm rather than the exception. Given these historical and, especially, contemporary dynamics, it seems possible that the demarcations between ecosystem regions may have grown less salient in learners' experiences of STEM selfefficacy and cultural sustenance - if nothing else, we must critically consider whether adjustments are necessary in how we conceive of and operationalize ecosystemic constructs when conducting research and planning educational experiences.

In addition to these possible ramifications, I feel it is worth returning to the series of analyses that did in fact yield statistically significant findings — specifically, those assessing the relationships between ecosystem areas and the outcomes associated with the Six strands of science learning. As noted earlier, the specific ecosystem region pairings that demonstrated statistically significant between mean six strands scale ranks were (a) home settings and school settings, (b) home settings and science center/museum settings, and (c) science center/museum settings and other site settings. Bearing in mind that the operationalization of the six strands that I employed in this study was, again, untested 
prior to the current implementation, these findings are nonetheless interesting to consider, as they suggest the possibility that the outcomes articulated by the six strands may be (roughly) equally achieved between the pairings not represented here. Of particular interest to me is the absence of statistical significance between science center/museum settings and school settings - in other words, between informal and formal built learning environments.

To reiterate, the six strands framework (NRC, 2009) was developed in response to an earlier framework of four strands of science learning that focused primarily upon learning occurring in formal education settings (NRC, 2007), with the goal of expanding the framework to include strands and outcomes that research and practice suggested would be particularly relevant to informal STEM learning environments. If, however, as this examination suggests, the six strands outcomes are holistically achieved at a relatively consistent level across formal and informal STEM learning spaces, it would necessitate a shift in thinking, certainly among informal STEM educators, and potentially among those operating in formal learning environments as well. Rather than imagining that formal and informal STEM education environments are varyingly supportive of strand outcomes (and developing learning experiences based on this assumption), educators and administrators would be called to consider the possibility that strand outcomes can be well-supported across the ecosystem and to teach and act accordingly. This possibility is further supported by the finding that the only one of the six strands items that demonstrated a statistically significant difference in distribution of responses across ecosystem type was strand 5 ("Engaging in scientific practices"), and then only between science center/museum settings and home settings. Perhaps, my research 
suggests, for all the time and thinking that has been dedicated to conceptualizing and exploring the differences between formal and informal learning spaces (and the outcomes associated with each), we may be more similar than not. This is not an altogether new idea - certainly there have been other researchers before me who have found evidence that both formal and informal learning settings have the potential to generate outcomes, albeit professional/adult outcomes, that can be linked to all six of the strands (Avraamidou, 2015; Carsten Conner \& Danielson, 2016). Given all this, it seems plausible, albeit in need of further research, that the conceptual divide between formal and informal STEM learning settings may be perceived (and, therefore, enacted and reified) primarily by educators, researchers, and administrators, rather than by learners themselves. It is possible, then, that from the perspective of learners as active and thoughtful agents who mindfully and intentionally navigate their STEM ecosystems, these distinctions may sometimes be relevant, but each ecosystem region has the potential to enrich and support STEM identity, engagement, and learning when the necessary conditions are met.

Lastly, with regard to the methodological and conceptual frameworks within which I conducted my research, I absolutely feel that the findings described above provide strong, albeit preliminary, support for the admittedly unconventional combinations and approaches I employed. The results of my statistical analyses highlight the complexity of teaching and learning within an ecosystemic framework, in the sense, to quote Mason (2009), that in the sense that "very large numbers of constituent elements or agents are connected to and interacting with each other in many different ways" (p. 118). Without the incorporation of a complexity lens, I might have been tempted to 
satisfy myself with overly simplistic operationalizations of variables and interpretations of findings, rather than seeking to illuminate the complex, systemic dynamics that are perpetually and inevitably at play in learners' experiences of agentic navigation of STEM ecosystems. At the same time, however, my analyses, and the entire foundations of the examination I have undertaken, would have been woefully inadequate without the inclusion of critical theory to foreground the ever-present influences of the systems of power and privilege that are woven throughout every aspect of STEM learning (and indeed of human existence, at least in the present day). Whereas complexity theory helps us to attend to the immense entanglement of people and systems, critical theory, as Peters (2005) stated,

must provide empirical and testable accounts of social conditions (focusing on the causes of oppression); it must aim toward change for the better, an alleviation of the human condition or 'emancipation'; and it must do so by providing a better self-understanding of the social agents who aim at transformation. (p. 38)

I have held these tenets of critical theory close to my heart while planning and undertaking this study, and while my contributions to our field's shared body of knowledge are admittedly modest, I am grateful that the inclusion of critical theory in my theoretical framework has allowed me, on some small scale, to advance each of these three goals. While I am far from the first researcher to recognize the complementariness of critical and complexity theory (see, for example, Cochran-Smith et al., 2014; Garlick, 2011; Marra, 2015), this pairing remains startlingly uncommon, and if this study can bring some measure of visibility to its immense potential, I consider this a meaningful outcome of my work. 
Just as my theoretical framework was a bit unorthodox, the same could be said for the methodology I employed, and likewise for my generation of largely untested instruments for the operationalization of my study variables. To the first of these two points, I am deeply grateful to my doctoral advisor and committee for so fully supporting my integration of a critical interpretive framework with my selected quantitative methodology to yield what I have termed a critical quantitative approach. This approach necessitated careful thought and continual self-reflection to ensure that I remained rigorous in my analysis while also honoring the legacy of critical methodologists before me by never losing sight of the human perspectives and experiences that the numbers in my dataset represent. As I noted earlier in this document, I fundamentally reject the notion that any methodology, quantitative or otherwise, is or can ever be divorced from the values, beliefs, assumptions, and perspectives of the researcher(s) who implement it. Rather, as eloquently stated by Ed Yong, Science Writer for the Atlantic, during his keynote address at the 2018 ASTC Conference in Hartford, Connecticut, "this is the beautiful lie of science: that it is an effective barrier against our own biases" (Yong, 2018). The belief that any number of procedures for "ensuring objectivity" can allow us as human beings to fully remove ourselves from the research we conduct is at best a wellintentioned falsehood, and at worst the cause of deep human pain and tragedy by virtue of its occlusion of questions left unanswered, paths left unexplored, and human experiences and voices inequitably silenced. By infusing my quantitative approach with the tenets of critical theorists and methodologists, my goal was to acknowledge and validate the inherently personal and value-laden nature of my (and all) research, and to use my own positionality and a visible starting point rather than presenting an imagined pseudo-reality 
in which it did not influence my asking of questions and interpretation of findings. This critical quantitative location is what I have grown to consider my methodological home, and while it will undoubtedly benefit from continued refinement over the years to come, I am gratified by richness and nuance it has offered me throughout this study.

In keeping with this, returning to my earlier point regarding instrumentation, I recognize that the scales I administered through my survey questionnaire were, with the exception of self-efficacy items, newly developed for my study and therefore untested in prior research. This being the case, in spite of my rigorous efforts to ground my new measures for culturally sustaining learning experiences (Appendix ) and the six strands of science learning in the respective bodies of literature related to these conceptual constructs, the potential undoubtedly existed for things to go horribly awry in the course of their inaugural implementation. Instead, I was deeply gratified to see that after some modifications to the CSLE scales to remove an item that appeared inconsistent with other item responses (potentially due to confusing wording), both scales, across all four ecosystem resource areas, demonstrated robust levels of internal consistency. It is my hope that I and other researchers will continue to test and refine these measures, and although I was unable to coordinate a review of the CSLE scale with Dr. Django Paris (as I hoped I might be able to do), this remains my goal in the months following the completion of the current study. Both the six strands and CSLE are deserving of further exploration in both formal and informal education settings-indeed, across the entire ecosystem — and the methodological approaches and resultant findings from my doctoral study will, I hope, offer a meaningful contribution to the field in service to such exploration. 


\section{Implications for Research and Practice}

To briefly reiterate the points introduced above, it is my hope that the present study provides relevant and useful findings as well as potential avenues for continued research by myself and others involved in the investigation of learning across STEM ecosystems, while concurrently suggesting changes in practice for STEM educators and administrators. First, speaking holistically, I urge my colleagues (and intend myself) to continue building and enriching the small but (hopefully) growing body of scholarship that pairs quantitative methodologies and associated methods with critical lenses for planning, administration, and interpretation of research. Such pairings unquestionably bring tensions and challenges, but to my mind, it is far better for us as researchers to be forced to confront, acknowledge, and carry these tensions with us through our work, rather than accepting the seductive but immensely harmful belief that any methodological approach can allow us to shed our positionality, biases and all. As Guba and Lincoln (2005) note, "the way in which we know is most assuredly tied up with both what we know and our relationships with our research participants (p. 209, emphasis in original), and these interconnections remain true and present irrespective of the methodologies we choose. It is my hope that my example, humble in scope though it may be, will encourage and empower others who recognize the potential and power of quantitative inquiry to offer insights into the human experience but are unwilling to align themselves with the unfortunate tenets of the quantitative tradition that suggest that true objectivity is achievable—or, indeed, desirable.

This study also holds both methodological and practical implications for STEM educators and leaders throughout the ecosystem in that my findings offer preliminary 
evidence that variation in outcomes can be observed and measured across ecosystem resource areas, as well as some possible avenues for instrumentation from a critical quantitative perspective. From national organizations (Stem Funders Network, 2016) to ISE researchers (Falk et al., 2016) to the federal government (NASA, 2021; National Science and Technology Council, 2018) and beyond, the past several years have seen exponential growth in the recognition of the fundamental importance of ecosystemic framing of STEM teaching and learning. However, my personal experience- and one of the primary motivators for my choice of STEM ecosystems as the focus of my doctoral research — has been that academic and practical ecosystemic explorations to date have been primarily if not exclusively approached with the implicit assumption that learners will passively react in certain ways if we, as educators and leaders, can simply find the right "triggers" to activate desired behavior. I wish to note here that I have the immense honor of holding deep, treasured personal and professional friendships with STEM educators and researchers working in both formal and informal settings, and these relationships lead me to feel certain that this framing, however problematic, is by no means malicious and is in fact employed with the best of intentions. By positioning the bulk of the agency with STEM educators and leaders, I acknowledge that we are called to recognize our own power and the significant ramifications of our choices and actions; this, I think, is all to the good, but it also unavoidably minimizes the agency of learners and the entirely legitimate reasons that might be held for navigating their STEM ecosystems in ways that, from the perspective of well-intentioned educators, may appear "sub-optimal." My study is intended to offer a reframing of this educator-centric approach, and to acknowledge and elevate learner voices and experiences from an agentic 
perspective, following in the tradition of dedicated, creative, and deeply caring

researchers and educators have come before (e.g., Cohen, 2020; Corin et al., 2017). From both a practical and theoretical standpoint, we require knowledge and tools that incorporate educator- and leader-centric perspectives as well as learner-centric narratives; my goal in this study is not to imply that only the latter hold value and utility, but to contribute to a rebalancing of what is, to date, a body of scholarship that is rather heavily skewed toward the former.

Returning to a point discussed in the preceding pages, it is worth reemphasizing the potential significance of these findings to suggest that further critical examination of the six strands of science learning framework may be warranted. In my personal experience as a member of the ISE field, I have collaborated on numerous projects and grant proposals that employed the six strands framework as an approach to organizing concepts, activities, and outcomes, and in nearly all (if not every) case, my colleagues and I accepted essentially without question the NRC's (2009) assertion that institutions such as ours are particularly well-positioned to support the first and sixth strands of science learning ("Developing interest in science" and "Identifying with the scientific enterprise," respectively). This is not to say, of course, that the outcomes associated with these strands are in any way anathema to science center and museum settings. There is, after all, ample evidence that ISE environments do encourage the development of interest related to STEM, as articulated in the first strand (Falk, Storksdieck, \& Dierking, 2007; Sample McMeeking et al., 2016), as well as fostering a sense of STEM identity, as articulated in the sixth strand (Pattison, Gontan, Ramos-Montañez, Shagott, Francisco, \& Dierking, 2020; Shein, Falk, \& Li, 2019). However, it is also fair and necessary to say 
that it is in many cases easier to abide by the generally accepted claims of strengths and weaknesses related to specific ecosystem resource areas than to critically question and engage in evidence-based assessments of the veracity and nuance of these claims. While I would certainly not position my current study as the authoritative examination of the application of the six strands of science learning across ecosystem areas, I do hope that my methodology and findings will offer new avenues for myself and others to continue exploring and refining this conceptual schema across future projects. As noted very early in this document, there are few frameworks that have been developed and implemented with informal STEM learning settings explicitly in mind, and I would never wish to suggest that this one is without utility; instead, with luck (and persistence), we will see it continue to evolve and be of use to educators and researchers for many years to come.

Finally, it is my great hope that this study will contribute to continued recognition of the value and promise of culturally sustaining pedagogy as an aspirational framework for educators and leaders working in both formal and informal STEM learning settings. Likewise, I hope that my choice of a critical quantitative methodology and my accompanying development of the Culturally Sustaining Learning Experiences Scale will motivate and energize others, researchers and practitioners alike, to continue applying and comprehensively assessing culturally sustaining approaches to STEM education. As I have likely made clear in the preceding pages, I feel strongly that culturally sustaining pedagogy holds immense promise as a framework to work toward the remedying of the deep historical and contemporary harms inflicted upon minoritized communities and learners by the educational system, and particularly by the STEM fields. In order to contribute to this educational framework reaching its full potential, however, it is 
incumbent upon us as educators, researchers, and leaders to develop and implement a range of methods and methodologies as we engage with learners across the ecosystem to attend to and elevate their voices and experiences. Perhaps unsurprisingly given its nature, much if not all of the small but growing body of scholarship related to culturally sustaining pedagogy (e.g., Kganetso, 2016; Kuttner, 2016; McCarty \& Lee, 2016; Nash et al., 2018; Weiland, 2015) has relied upon qualitative methodologies. Such methodologies, and the narratives of learning and cultural sustenance they illuminate, are vitally important and deeply meaningful. However, it is my heartfelt belief that in order for a more complete picture to be painted, we must begin to develop and critically refine accompanying quantitative approaches to explore and describe the cultural sustenance of learners in STEM fields. So long as we never allow ourselves to forget that every number, every quantitative datum, represents the perspective and experience of a person who has granted us the incredible privilege of telling their stories, the employment of critical quantitative methodologies and methods has great potential to support and enrich this burgeoning area of research and practice. I am grateful and humbled to have the opportunity to play some small part in this enrichment, and urge myself and others to pick up the torch and continue refining the instrument and methodological approach I piloted in my study.

\section{Conclusion}

As this research project draws to an end, I find myself overwhelmed with gratitude for the opportunity I have been granted to dedicate such deep and focused attention to the examination (and, hopefully, some small measure of redress) of a problem of educational practice that is close to my own heart. The field of STEM 
education, spanning informal and formal learning environments alike, is, in my experience, overwhelmingly populated by professionals who are fiercely dedicated to principles of equitable and sustaining pedagogy, and who strive to put these principles into practice every day of their lives. Yet, in spite of this, we all too often fail to conceive of learners at the center of their journeys across and throughout their ecosystems, and in so doing, we develop and reinforce educational models that unavoidably perpetuate systemic inequities in opportunity access and learning outcomes. By taking an ecosystemic perspective, we recognize that learners are making choices that are sensible and legitimate based on their own perspectives and lived experiences; the findings from my study lend some preliminary credence to the notion that when looking across ecosystem areas, learners may be availing themselves of resources and opportunities such that inequities in self-efficacy and cultural sustenance are minimized. Taking this as a cue, we have then before us a clear responsibility—no, not to attempt to draw every individual learner to engage with the experiences we design regardless of personal interests or desires, but rather to weave threads of cultural sustenance into all that we do; to build awareness of and eliminate barriers, whether financial, perceptual, or cultural, to access STEM throughout our ecosystems; and always, at every opportunity, to seek out, respond to, and honor the voices and experiences of learners, their families, and their communities.

Through my doctoral study, and in collaboration with learners and educators throughout Oregon's South Willamette Valley, it has been my great honor to offer a humble contribution to the efforts of scholars, educators, and activists who have come before, who work alongside me, and who will follow in the years ahead. As is often the 
case with research regarding the human experience, this exploration has yielded more questions than answers; many paths of inquiry lead not to satisfying and tidy conclusions, but to further forks in the road and trails of curiosity to explore. This is, to my mind, all to the good, for what would life be without curiosity, without unexplored turns in the road and uncharted corners of the map to compel us to further our understanding of this complex, flawed, but unimaginably beautiful world we share? Our goal should be always to contribute to a world that is, in some small way, richer in justice, equity, and light than we found it, but we cannot ever allow ourselves to forget that to achieve this goal requires that we work in community with and learn from each other; no one of us alone can hope to offer answers that benefit us all. The work is not done, and likely will never be so, but with each step that is taken to put ourselves in authentic and sustaining service to those who grant us the privilege and joy of being a part of their journeys of learning, we build the foundation for a hopeful future in which our ecosystems are free of barriers for engagement by those who seek knowledge and fulfillment. As we carry these ideals with us and allow them to guide our actions as educational leaders, we draw ever closer to a day on which we need no longer question whether our children, and other children we may never meet but whose care and sustenance are no less important, will find every pathway of learning they may ever wish to explore open to them, awaiting the impressions of eager feet set alight by the joy of new discoveries. 


\section{References}

Ackoff, R. L. (1974). Redesigning the future: A systems approach to societal problems. New York, NY: Wiley.

Adair, J. K. (2014). Agency and expanding capabilities in early grade classrooms: What it could mean for young children. Harvard Educational Review, 84(2), 217-242.

Affuso, G., Bacchini, D., \& Miranda, M. C. (2017). The contribution of school-related parental monitoring, self-determination, and self-efficacy to academic achievement. The Journal of Educational Research, 110(5), 565-574. doi:10.1080/00220671.2016.1149795

Afterschool Alliance. (n.d.). General lobbying rules for non profit organizations. Retrieved from http://www.afterschoolalliance.org/documents/Toolbox/General\%20Lobbying\%2 0Rules\%20for\%20NonProfit\%20Orgs\%2008\%2014\%2008.pdf

AL-Baddareen, G., Ghaith, S., \& Akour, M. (2015). Self-efficacy, achievement goals, and metacognition as predictors of academic motivation. Procedia-Social and Behavioral Sciences, 191, 2068-2073. doi:10.1016/j.sbspro.2015.04.345

Alim, H. S., \& Paris, D. (2017). What is culturally sustaining pedagogy and why does it matter? In D. Paris \& H. S. Alim (Eds.), Culturally sustaining pedagogies: Teaching and learning for justice in a changing world (pp. 1-21). New York, NY: Teachers' College Press.

Allan, E. J., Iverson, S. V., \& Roper-Huilman, R. (Eds., 2010). Reconstructing policy in higher education. New York, NY: Routledge. 
Allen, S. (2004). Designs for learning: Studying science museum exhibits that do more than entertain. Science Education, 88(4), S17-S33.

Alqahtani, A. Y., \& Rajkhan, A. A. (2020). E-learning critical success factors during the COVID-19 pandemic: A comprehensive analysis of e-learning managerial perspectives. Education Sciences, 10(9), 216-231. doi:10.3390/educsci10090216

Alvaro, C., Jackson, L. A., Kirk, S., McHugh, T. L., Hughes, J., Chircop, A., \& Lyons, R. F. (2011). Moving Canadian governmental policies beyond a focus on individual lifestyle: Some insights from complexity and critical theories. Health Promotion International, 26(1), 91-99. doi:10.1093/heapro/daq052

Anderson, G. (1989). Critical ethnography in education: Origins, current status and new directions. Review of Educational Research, 59(3), 249-270.

Arguel, A., Lockyer, L., Lipp, O. V., Lodge, J. M., \& Kennedy, G. (2016). Inside out: Detecting learners' confusion to improve interactive digital learning environments. Journal of Educational Computing, 55(4), 526-551. doi: $10.1177 / 0735633116674732$

Aronowitz, S., \& Giroux, H. (1985). Education under siege. South Hadley, MA: Bergin \& Harvey.

Association of Science-Technology Centers (ASTC). (2013). 2013 science center and museum indicators. Retrieved from http://www.astc.org/wpcontent/uploads/2014/10/2013-Science-Center-Statistics.pdf

Association of Science-Technology Centers (ASTC). (2016). About science centers. Retrieved from http://www.astc.org/about-astc/about-science-centers/ 
Avraamidou, L. (2015). Reconceptualizing elementary teacher preparation: A case for informal science education. International Journal of Science Education, 37(1), 108-135. doi:10.1080/09500693.2014.969358

Babbie, E. R. (1990). Survey research methods ( $2^{\text {nd }}$ ed.). Belmont, CA: Wadsworth, Cengage Learning.

Babbie, E. R. (2016). The practice of social research (14 ${ }^{\text {th }}$ ed.). Boston, MA: Cengage Learning.

Bandura, A. (1971). Social learning theory. New York, NY: General Learning Press.

Bandura, A. (1977). Self-efficacy: Toward a unifying theory of behavioral change. Psychological Review, 84(2), 191-215.

Bandura, A. (1982). Self-efficacy mechanism in human agency. American Psychologist, 37(2), 122-147. doi:10.1037/0003-066X.37.2.122

Bandura, A. (1986a). Social foundations of thought and action: A social cognitive theory. Englewood Cliffs, NJ: Prentice-Hall.

Bandura, A. (1986b). The explanatory and predictive scope of self-efficacy theory. Journal of Social and Clinical Psychology, 4, 359-373. doi:10.1521/jscp.1986.4.3.359

Bandura, A. (1992). Social cognitive theory. In R. Vasta (Ed.), Six theories of child development: Revised formulations and current issues (pp. 1-60). London, England: Jessica Kingsley.

Bandura, A. (2006a). Guide for constructing self-efficacy scales. In F. Pajares \& T. Urdan (Eds.), Self-efficacy beliefs of adolescents (Vol. 5., pp. 307-337). Greenwich, CT: Information Age. 
Bandura, A. (2006b). Toward a psychology of human agency. Perspectives on Psychological Science, 1(2), 164-180.

Bandura, A. (2011). Social cognitive theory. In P. A. M. van Lange, A. W. Kruglanski, \& E. T. Higgins (Eds.), Handbook of social psychological theories (pp. 349-373). London, England: SAGE.

Bandura, A., Barbaranelli, C., Caprara, G. V., \& Pastorelli, C. (1996). Multifaceted impact of self-efficacy beliefs on academic functioning. Child Development, 67(3), 1206-1222.

Bandura, A., Barbaranelli, C., Caprara, G. V., \& Pastorelli, C. (2001). Self-efficacy beliefs as shapers of children's aspirations and career trajectories. Child Development, 72(1), 187-206. doi:10.1111/1467-8624.00273.

Bandura, A., \& Locke, E. A. (2003). Negative self-efficacy and goal effects revisited. Journal of Applied Psychology, 88(1), 87-99. doi:10.1037/0021-9010.88.1.87

Barth, J. M., Guadagno, R. E., Rice, L., Eno, C. A., \& Minney, J. A. (2015). Untangling life goals and occupational stereotypes in men's and women's career interest. Sex Roles, 73(11), 502-518. doi:10.1007/s11199-015-0537-2

Basile, V., \& Lopez, E. (2015). And still I see no changes: Enduring views of students of color in science and mathematics education policy reports. Science Education, 99, 519-548. doi:10.1002/sce.21156

Bevan, D., \& Gitsham, M. (2009) Context, complexity and connectedness: Dimensions of globalization revealed. Corporate Governance: The International Journal of Business in Society, 9(4), 435-447. doi:10.1108/14720700910984981 
Bevc, C. A., Young, D., \& Peterman, K. (2016). Using social network analysis to document science festival partnerships. JCOM: Journal of Science Communication, 15(5), 1-15.

Bewick, V., Cheek, L., \& Ball, J. (2004). Statistics review 10: further nonparametric methods. Critical care (London, England), 8(3), 196-199. doi:10.1186/cc2857

Bjørnebekk, G., Diseth, Å., \& Ulriksen, R. (2013). Achievement motives, self-efficacy, achievement goals, and academic achievement at multiple stages of education: A longitudinal analysis. Psychological Reports: Human Resources \& Marketing, 112(3), 771-787. doi:10.2466/14.09.PR0.112.3.771-787

Blalock, N. R. (2013). Culturally sustaining pedagogies: Understanding school practices and the academic achievement of American Indian and Alaska Native students through a multidisciplinary lens (Doctoral dissertation). Retrieved from ProQuest. (UMI \#3602004)

Bong, M. (2001). Between- and within-domain relations of academic motivation among middle and high school students: Self-efficacy, task-value, and achievement goals. Journal of Educational Psychology, 93(1), 23-34.

Boon, H. J., \& Lewthwaite, B. (2015). Development of an instrument to measure a facet of quality teaching: Culturally responsive pedagogy. International Journal of Educational Research, 72, 38-58. doi:10.1016/j.ijer.2015.05.002

Bowen, M. L., \& Tillman, A. S. (2015). Developing culturally responsive surveys: Lessons in development, implementation, and analysis from Brazil's African descent communities. American Journal of Evaluation, 36(1), 25-41. doi:10.1177/1098214014539831 
Brack, G., Lassiter, P. S., Hill, M. B., \& Moore, S. A. (2011). Ecosystemic complexity theory of conflict: Understanding the fog of conflict. The Journal of Humanistic Counseling, 50, 3-15. doi:10.1002/j.2161-1939.2011.tb00102.x

Bronner, S. E. (2011).Critical theory: A very short introduction. Oxford, NY: Oxford University Press.

Bruce, B. (2002). A friendly, welcoming attitude toward change. Journal of Adolescent \& Adult Literacy, 45(7), 590-595.

Butt, G. (2010). Perspectives on research in geography education. International Research in Geographical and Environmental Education, 19(2), 79-82. doi:10.1080/10382046.2010.482179

Caprara, G. V., Vecchione, M., Alessandri, G., Gerbino, M., \& Barbaranelli, C. (2010). The contribution of personality traits and self-efficacy beliefs to academic achievement: A longitudinal study. British Journal of Psychology, 81(1), 78-96. doi:10.1348/2044-8279.002004

Carlile, A. (2012). 'Critical bureaucracy' in action: Embedding student voice into school governance. Pedagogy, Culture \& Society, 20(3), 393-412. doi:10.1080/14681366.2012.712053

Carsten Conner, L. D., \& Danielson, J. (2016). Scientist role models in the classroom: How important is gender matching? International Journal of Science Education, 38(15), 2414-2430. doi:10.1080/09500693.2016.1246780

Ceci, S. J., \& Williams, W. M. (2011). Understanding current causes of women's underrepresentation in science. Proceedings of the National Academy of Sciences, 108(8), 3157-3162. doi:10.1073/pnas 
Ceci, S. J., Williams, W. M., \& Barnett, S. M. (2009). Women's underrepresentation in science: Sociocultural and biological considerations. Psychological Bulletin, 135(2), 218-261. doi:10.1037/a0014412

Chase, M. M., Dowd, A. C., Pazich, L. B., \& Bensimon, E. M. (2014). Transfer equity for "minoritized" students: A critical policy analysis of seven states. Educational Policy, 28(5), 669-717. doi:10.1177/0895904812468227

Chen, G., Gully, S. M., \& Eden, D. (2001). Validation of a new general self-efficacy scale. Organizational Research Methods, 4(1), 62-83. doi: $10.1177 / 109442810141004$

Cochran-Smith, M., Ell, F., Grudnoff, L., Ludlow, L., Haigh, M., \& Hill, M. (2014). When complexity theory meets critical realism: A platform for research on initial teacher education. Teacher Education Quarterly, 41(1), 105-122.

Cody, S. (2010). Fostering a lifelong love of science outside the classroom. Retrieved from https://www.nasw.org/article/fostering-lifelong-love-science-outsideclassroom

Cohen, B. J. (2020). Amplifying Latinx voice through interview study: Highlighting mathematics identity as a pathway to student self-empowerment and academic agency (Doctoral dissertation). Retrieved from ProQuest. (UMI \#27832403)

Cook, L., Mason, M., Morse, R., \&Neuhauser, A. (2015, June 29). The 2015 U.S. news/Raytheon STEM index. U.S. News and World Report. Retrieved from http://www.usnews.com/news/stem-index/articles/2015/06/29/the-2015-us-newsraytheon-stem-index 
Corin, E. N., Gail, J. M., Andre, T., Childers, G. M., \& Stevens, V. (2017). Science hobbyists: Active users of the science-learning ecosystem. International Journal of Science Education, 7(2), 161-180.

Cornelius, L. J., \& Harrington, D. (2014). A social justice approach to survey design and analysis. New York, NY: Oxford University Press.

Coutinho, S. A., \& Neuman, G. (2008). A model of metacognition, achievement goal orientation, learning style and self-efficacy. Learning Environments Research, 11, 131-151. doi:10.1007/s10984-008-9042-7

Creswell, J. W. (2014). Research design: Qualitative, quantitative, and mixed methods approaches. Los Angeles, CA: SAGE.

Cross, J. (2006). Informal learning: Rediscovering the natural pathways that inspire innovation and performance. San Francisco, CA: Wiley.

Crowl, M., Devitt, A., Jansen, H., Zee, E., \& Winograd, K. (2013). Encouraging prospective teachers to engage friends and family in exploring physical phenomena. Journal of Science Teacher Education, 24(1), 93-110. doi:10.1007/s10972-012-9310-3

Crusio, W. E., Rubino, C., \& Delprato, A. (2017). Engaging high school students in systems biology through an e-internship program. F1000 Research, 6, 20-26. doi:10.12688/f1000research.10570.1

Cudworth, E., \& Hobden, S. (2012). The foundations of complexity, the complexity of foundations. Philosophy of the Social Sciences, 42(2), 163-187. doi: $10.1177 / 0048393110388038$ 
Davis, B., \& Sumara, D. (2008). The death and life of great educational ideas: Why we might want to avoid a critical complexity theory. Journal of the Canadian Association for Curriculum Studies, 6(1), 163-176.

Dawson, E. (2014). "Not designed for us": How science museums and science centers socially exclude low-income, minority ethnic groups. Science Education, 98(6), 981-1008. doi:10.1002/sce.21133

Dawson, E. (2018). Reimagining publics and (non)participation: Exploring exclusion from science communication through the experiences of low-income, minority ethnic groups. Public Understanding of Science, 27(7), 772-786. doi:10.1177/0963662517750072

De Laat, M., \& Lally, V. (2003). Complexity, theory and praxis: Researching collaborative learning and tutoring processes in a networked learning community. Instructional Science, 31, 7-39. doi:10.1023/A:1022596100142

Delgado Bernal, D., \& Villalpando, O. (2016). An apartheid of knowledge in academia: The struggle over the "legitimate" knowledge of faculty of color. In E. Taylor, D. Gillborn, \& G. Ladson-Billings (Eds.), Foundations of critical race theory in education ( $2^{\text {nd }}$ ed., pp. 77-92). New York, NY: Routledge.

Diekman, A. B., Brown, E. R., Johnston, A. M., \& Clark, E. K. (2010). Seeking congruity between goals and roles: A new look at why women opt out of science, technology, engineering, and math careers. Psychological Science, 21(8), 10511057. doi:10.1177/0956797610377342. 
Diekman, A. B., Clark, E. K., Johnston, A. M., Brown, E. R., \& Steinberg, M. (2011). Malleability in communal goals and beliefs influences attraction to STEM careers: Evidence for a goal congruity perspective. Journal of Personality and Social Psychology, 101(5), 902-918. doi:10.1037/a0025199.

Diem, S., Young, M. D., Welton, A. D., Mansfield, K. C., \& Lee, P.-L. (2014). The intellectual landscape of critical policy analysis. International Journal of Qualitative Studies in Education, 27(9), 10681090.doi:10.1080/09518398.2014.916007

Dimick, A. S. (2016). Exploring the potential and complexity of a critical pedagogy of place in urban science education. Science Education, 100, 814-836. doi:10.1002/sce.21233

Domínguez, S., Weffer, S. E., \& Embrick, D. G. (2020). White sanctuaries: White supremacy, racism, space, and fine arts in two metropolitan museums. American Behavioral Scientist, 64(14), 2028-2043. doi:10.1177/0002764220975077

Doran, P. R. (2017). Teachers' self-reported knowledge regarding English learners: Perspectives on culturally and linguistically inclusive instruction and intervention. International Journal of Inclusive Education, 21(5), 557-572. doi:10.1080/13603116.2016.1218949

Doucet, F. (2017). What does a culturally sustaining learning climate look like? Theory Into Practice, 56(3), 195-204. doi:10.1080/00405841.2017.1354618

Duffy, K., \& Scott, A. P. (1998). Viewing an old issue through a new lens: A critical theory insight into the education-practice gap. Nurse Education Today, 18, 183189. 
Eagly, A. H., Wood, W., \& Diekman, A. B. (2000). Social role theory of sex differences and similarities: A current appraisal. In T. Eckes \& H. M. Trautner (Eds.), The developmental psychology of gender (pp. 123-173). Mahwah, NJ: Erlbaum.

Eoyang, G., \& Holladay, R. (2013). Adaptive action: Leveraging uncertainty in your organization. Redwood City, CA: Stanford Business Books.

Evans, C. D., \& Diekman, A. B. (2009). On motivated role selection: Gender beliefs, distant goals, and career interest. Psychology of Women Quarterly, 33(2), 235249. doi:10.1111/j.1471-6402.2009.01493.x.

Falk, J., \& Dierking, L. (2010). The 95 percent solution: School is not where most Americans learn most of their science. The American Scientist, 98(6), 486-493.

Falk, J. H., Dierking, L. D., Swanger, L. P., Staus, N., Back, M., Barriault, C.,...Verheyden, P. (2016). Correlating science center use with adult science literacy: An international, cross-institutional study. Science Education, 100(5), 849-876. doi:10.1002/sce.21225

Falk, J., Randol, S., \& Dierking, L. (2012). Mapping the informal science education landscape: An exploratory study. Public Understanding of Science, 21(7), 865874.

Fenwick, T. (2012) Complexity science and professional learning for collaboration: A critical reconsideration of possibilities and limitations. Journal of Education and Work, 25(1), 141-162. doi:10.1080/13639080.2012.644911

Ferguson, C. J., \& Heene, M. (2012). A vast graveyard of undead theories: Publication bias and psychological science's aversion to the null. Perspectives on Psychological Science, 7(6), 555-561. doi:10.1177/1745691612459059 
Field, A. P. (2018). Discovering statistics using IBM SPSS statistics $\left(5^{\text {th }}\right.$ ed., North American edition). Thousand Oaks, CA: SAGE.

Firth, R., \& Morgan, J. (2010). What is the place of radical/critical research in geography education? International Research in Geographical and Environmental Education, 19(2), 109-113. doi:10.1080/10382046.2010.482193

Fleet Science Center. (2018). STEM ecosystem. Retrieved from http://www.rhfleet.org/stem-ecosystem

Ford, D. J., Brickhouse, N.W., Lottero-Perdue, P., \& Kittleson, J. M. (2006). Elementary girls' science reading at home and school. Science Education, 90(2), 270-288. doi:10.1002/sce.20139

Fouad, N. A., \& Santana, M. C. (2017). SCCT and underrepresented populations in STEM fields: Moving the needle. Journal of Career Assessment, 25(1), 24-39. doi: $10.1177 / 1069072716658324$

Fowler, F. J. (2014). Survey research methods (5 $5^{\text {th }}$ ed.). Thousand Oaks, CA: SAGE. Gair, S. (2018). Upholding social justice: Obligation or optional extra in social work? Critical reflections on a classroom-based inquiry. Journal of Ethnic \& Cultural Diversity in Social Work, 27(2), 140-156. doi:10.1080/15313204.2017.1409674

Garlick, S. (2011). Complexity, masculinity and critical theory: Revisiting Marcuse on technology, Eros, and nature. Critical Sociology, 39(2), 223-238. doi:10.1177/0896920511421032

Gerber, A. S., \& Malhotra, N. (2008). Publication bias in empirical sociological research: Do arbitrary significance levels distort published results? Sociological Methods \& Research, 37(1), 3-30. doi:10.1177/0049124108318973 
Gillborn, D. (2016). Education policy as an act of white supremacy. In E. Taylor, D. Gillborn, \& G. Ladson-Billings (Eds.), Foundations of critical race theory in education ( $2^{\text {nd }}$ ed., pp. 43-59). New York, NY: Routledge.

Gliem, J. A., \& Gliem, R. R. (2017). Calculating, interpreting, and reporting Cronbach's alpha reliability coefficient For Likert-type scales [Paper presentation]. 2003 Midwest Research to Practice Conference in Adult, Continuing, and Community Education, Columbus, OH. Retrieved from https://scholarworks.iupui.edu/bitstream/handle/1805/344/Gliem\%20\%26\%20Gli em.pdf

Goodkind, S. (2013). Single-sex public education for low-income youth of color: A critical theoretical review. Sex Roles, 69, 393-402. doi:10.1007/s11199-0120219-2

Gould, J. A. (2011). Does it really take a village to raise a child (or just a parent?): An examination of the relationship between the members of the residence of a middle-school student and the student's satisfaction with school. Education, 132(1), 28-38.

Gran, A.-B., Vestberg, N. L., Booth, P., \& Ogundipe, A. (2019). A digital museum's contribution to diversity - a user study. Museum Management and Curatorship, 34(1), 58-78. doi:10.1080/09647775.2018.1497528

Grand-Clement, S., Devaux, A., Belanger, J., \& Manville, C. (2017). Digital learning: Education and skills in the digital age. Santa Monica, CA: RAND Corporation and Corsham Institute. Retrieved from https://www.rand.org/pubs/conf_proceedings/CF369.html 
Greenwood, D. (2010). A critical analysis of sustainability education in schooling's bureaucracy: Barriers and small openings in teacher education. Teacher Education Quarterly, 37(4), 139-154.

Grice, J. W., Craig, D. P. A., \& Abramson, C. I. (2015). A simple and transparent alternative to repeated measures ANOVA. SAGE Open, 5(3), 1-13. doi: $10.1177 / 2158244015604192$

Grincheva, N. (2014). The online museum: A 'placeless' space of the 'civic laboratory.' Museum Anthropology Review, 8(1), 1-21. doi:10.14434/mar.v8i1.3187

Guba, E. G., \& Lincoln, Y. S. (2005). Paradigmatic controversies, contradictions, and emerging confluences. In N.K. Denzen \& Y.S. Lincoln (Eds.), The SAGE handbook of qualitative research ( $3^{\text {rd }}$ ed.), (pp. 191-215). Thousand Oaks, CA: SAGE.

Han, H. S. (2010). Sociocultural influence on children's social competence: A close look at kindergarten teachers' beliefs. Journal of Research in Childhood Education, 24(1), 80-96. doi:10.1080/02568540903439425

Hanson, S. L. (2004). African American women in science: Experiences from high school through the postsecondary years and beyond. NWSA Journal, 16, 96-115.

Hardin, E. E., \& Longhurst, M. O. (2016). Understanding the gender gap: Social cognitive changes during an introductory STEM course. Journal of Counseling Psychology, 63(2), 233-239. doi:10.1037/cou0000119

Harding, S. G. (1998). Is science multicultural?: Postcolonialisms, feminisms, and epistemologies. Bloomington: Indiana University Press. 
Hardy, I., \& Woodcock, S. (2015). Inclusive education policies: Discourses of difference, diversity and deficit. International Journal of Inclusive Education, 19(2), 141164. doi:10.1080/13603116.2014.908965

Henze, R., \& Arriaza, G. (2006). Language and reforming schools: A case for a critical approach to language in educational leadership. International Journal of Leadership in Education, 9(2), 157-177. doi:10.1080/13603120600697387

Horn, J. (2008). Human research and complexity theory. Educational Philosophy and Theory, 40(1), 130-143. doi:10.1111/j.1469-5812.2007.00395.x

Hsiao, Y.-J. (2015). The culturally responsive teacher preparedness scale: An exploratory study. Contemporary Issues in Education Research, 8(4), 241-250.

Huang, C. (2016). Achievement goals and self-efficacy: A meta-analysis. Educational Research Review, 19, 119-137. doi:10.1016/j.edurev.2016.07.002

Hudson, R. C., Duncan, S., \& Reeve, C. (2016). Affinity spaces for informal science learning: Developing a research agenda. St. Paul, MN: TPT Twin Cities Public Television.

Internal Revenue Service. (2016). The restriction of political campaign intervention by section 501(c)(3) tax-exempt organizations. Retrieved from https://www.irs.gov/charities-non-profits/charitable-organizations/the-restrictionof-political-campaign-intervention-by-section-501-c-3-tax-exempt-organizations Jeffers, C. S. (2003). Museum as process. The Journal of Aesthetic Education, 37(1), 107-119. doi:10.1353/jae.2003.0005 
Jiang, S., Simpkins, D. S., \& Eccles, J. S. (2020). Individuals' math and science motivation and their subsequent STEM choices and achievement in high school and college: A longitudinal study of gender and college generation status differences. Developmental Psychology, 56(11), 2137-2151. doi:10.1037/dev0001110

Jiang, Y., Song, J., Lee, M., \& Bong, M. (2014) Self-efficacy and achievement goals as motivational links between perceived contexts and achievement. Educational Psychology, 34(1), 92-117. doi:10.1080/01443410.2013.863831

Jones, M. G., Howe, A., \& Rua, M. J. (2000). Gender differences in students' experiences, interests, and attitudes toward science and scientists. Science Education, 84(2), 180-192. doi:10.1002/(SICI)1098237X(200003)84:2<180::AID-SCE3>3.0.CO;2-X.

Jörg, T., Davis, B., \& Nickmans, G. (2007).Towards a new, complexity science of learning and education. Educational Research Review, 2(2), 145-156. doi:10.1016/j.edurev.2007.09.002

Journal Staff. (2017). States add more STEM learning ecosystems. Retrieved from https://thejournal.com/articles/2017/05/25/states-add-17-more-stem-learningecosystems.aspx

Kganetso, L. M. W. (2016). Creating and using culturally sustaining informational texts. The Reading Teacher, 70(4), 445-455. doi:10.1002/trtr.1546 
Kitchen, J. A., Sonnert, G., \& Sadler, P. M. (2018). The impact of college- and university-run high school summer programs on students' end of high school STEM career aspirations. Science Education, 102(3), 529-547. doi:10.1002/sce.21332

Klingner, J. K., \& Boardman, A. G. (2011). Addressing the "research gap" in special education through mixed methods. Learning Disability Quarterly, 34(3), 208218. doi:10.1177/07319487114I7S59

Krishnamurthi, A., \& Rennie, L. J. (n.d.). Informal science learning and education: Definitions and goals. Retrieved from http://afterschoolalliance.org/documents/STEM/Rennie_Krishnamurthi.pdf

Kuttner, P. J. (2016). Hip-hop citizens: Arts-based, culturally sustaining civic engagement pedagogy. Harvard Educational Review, 86(4), 527-555.

Ladson-Billings, G. (1995). Toward a theory of culturally relevant pedagogy. American Educational Research Journal, 32(3), 465-491.

Ladson-Billings, G. (2014). Culturally relevant pedagogy 2.0: a.k.a. the remix. Harvard Educational Review, 84(1), 74-84.

Lee, C. D. (2017). An ecological framework for enacting culturally sustaining pedagogy. In D. Paris \& H. S. Alim (Eds.), Culturally sustaining pedagogies: Teaching and learning for justice in a changing world (pp. 261-273). New York, NY: Teachers' College Press.

LeGrand, J. (2013). Exploring gender differences across elementary, middle, and high school students' science and math attitudes and interest (Doctoral dissertation). Retrieved from ProQuest. (UMI \#3556932) 
Lin, L., \& Chu, H. (2018). Quantifying publication bias in meta-analysis. Biometrics, 74(3), 785-794. doi:10.1111/biom.12817

Malcom, L. E. (2010). Charting the pathways to STEM for Latina/o students: The role of community colleges. New Directions for Institutional Research, 148, 29-40. doi:10.1002/ir.359

Maltese, A. V., \& Tai, R. H. (2010). Eyeballs in the fridge: Sources of early interest in science. International Journal of Science Education, 32(5), 669-685. doi:10.1080/09500690902792385

Mansfield, K. C., Welton, A. D., \& Grogan, M. (2014). "Truth or consequences”: A feminist critical policy analysis of the STEM crisis. International Journal of Qualitative Studies in Education, 27(9), 1155-1182.

doi:10.1080/09518398.2014.916006

Marra, M. (2015). Cooperating for a more egalitarian society: Complexity theory to evaluate gender equity. Evaluation, 21(1), 32-46.

doi: $10.1177 / 1356389014564856$

Marsh, H. W. (2007). Self-concept theory, measurement and research into practice: The role of self-concept in educational psychology. Leicester, England: British Psychological Society.

Mason, M. (2008). What is complexity theory and what are its implications for educational change? Educational Philosophy and Theory, 40(1), 35-49. doi:10.1111/j.1469-5812.2007.00413.x 
Mason, M. (2009). Making educational development and change sustainable: Insights from complexity theory. International Journal of Educational Development, 29(2), 117-124. doi:10.1016/j.ijedudev.2008.09.005

Mason, M. (2014). Complexity theory in education governance: Initiating and sustaining systemic change. Paper presented at the Governing Complex Education Systems (GCES): Understanding complexity: The future of education governance, Ministry of Education and Research, Oslo, Norway.

Mason, M. (2016). Is thorough implementation of policy change in education actually possible? What complexity theory tells us about initiating and sustaining change. European Journal of Education, 51(4), 437-440. doi:10.1111/ejed.12193

McCarty, T. L., \& Lee, T. S. (2014). Critical culturally sustaining/revitalizing pedagogy and indigenous education sovereignty. Harvard Educational Review, 84(1), 101124.

McQuillan, P. J. (2008). Small-school reform through the lens of complexity theory: It's “good to think with.” Teachers College Record, 110(9), 1772-1801.

Merolla, D. M. (2017). Self-efficacy and academic achievement: The role of neighborhood cultural context. Sociological Perspectives, 60(2), 378-393. doi:10.1177/0731121416629993

Mertens, D. M. (2010a). Philosophy in mixed methods teaching: The transformative paradigm as illustration. International Journal of Multiple Research Approaches, 4(1), 9-18.

Mertens, D. M. (2010b). Transformative mixed methods research. Qualitative Inquiry, 16(6), 469-474. doi:10.1177/1077800410364612 
Midgley, C., Maehr, M. L., \& Urdan, T. (1993). Manual for the patterns of adaptive learning survey (PALS). Ann Arbor: University of Michigan.

Mincarelli, P. (2015). Building a STEM education ecosystem in Baltimore. Retrieved from https://www.huffingtonpost.com/paul-mincarelli/building-a-stemeducation_b_8401706.html

Mittwede, S. K. (2012). Research paradigms and their use and importance in theological inquiry and education. Journal of Education \& Christian Beliefs, 16(1), 23-40.

Mladenova, T., Kalmukov, Y., \& Valova, I. (2020). Covid 19 - A major cause of digital transformation in education or just an evaluation test. TEM Journal, 9(3), 11631170. doi:10.18421/TEM93-42

Moll, L. C., Amanti, C., Neff, D., \& Gonzalez, N. (1992). Funds of knowledge for teaching: Using a qualitative approach to connect homes and classrooms. Theory Into Practice, $\mathrm{XXXI(2),132-141.}$

Morçöl, G. (2010). Issues in reconceptualizing public policy from the perspective of complexity theory. E:CO, 12(1), 52-60.

Morgan, S. E. (2002). From numbers to words: Reporting statistical results for the social sciences. Boston, MA: Allyn and Bacon.

Nardi, P. M. (2018). Doing survey research: A guide to quantitative methods $\left(4^{\text {th }}\right.$ Ed.). New York, NY: Routledge.

Nash, K., Panther, L., \& Arce-Boardman, A. (2018). La historia de mi nombre: A culturally sustaining early literacy practice. The Reading Teacher, 71(5), 605609. doi:10.1002/trtr.1665 
National Aeronautics and Space Administration (NASA). (2021). STEM ecosystems.

NASA Science. Retrieved from https://science.nasa.gov/science-activationteam/stem-ecosystems

National Research Council (NRC). (2007). Taking science to school: Learning and teaching science in grades $K-8$. Washington, DC: The National Academies Press.

National Research Council (NRC). (2009). Learning science in informal environments: People, places, and pursuits. Washington, DC: The National Academies Press. National Science and Technology Council (NSTC). (2018). Charting a course for success: America's strategy for STEM education. Executive Office of the President of the United States, Office of Science and Technology Policy. Retrieved from https://www.energy.gov/sites/default/files/2019/05/f62/STEMEducation-Strategic-Plan-2018.pdf

National Science Board (NSB). (2014). Science and engineering indicators 2014 (NSD 14-01). Retrieved from https://www.nsf.gov/statistics/seind14/

National Science Board (NSB). (2016). Science and engineering indicators 2016(NSB2016-1). Retrieved from https://www.nsf.gov/statistics/2016/nsb20161/

National Science Foundation (NSF). (2015). Women, minorities, and persons with disabilities in science and engineering: 2015 digest. Retrieved from https://www.nsf.gov/statistics/2015/nsf15311/digest/nsf15311-digest.pdf 
National Science Foundation, National Center for Science and Engineering Statistics (NSF NCSES). (2017). Women, minorities, and persons with disabilities in science and engineering: 2017.Special Report NSF 17-310. Arlington, VA: Author. Retrieved from https://www.nsf.gov/statistics/2017/nsf17310/static/downloads/nsf17310digest.pdf

Ngo, H. V. (2012). Cultural competence in Alberta schools: Perceptions of ESL families in four major school boards. TESL Canada Journal, 29(6), 204-223.

Nordin, A. (2014). Crisis as a discursive legitimation strategy in educational reforms: A critical policy analysis. Education Inquiry, 5(1), 109-126. doi:10.3402/edui.v5.24047

Oregon Museum of Science and Industry (OMSI). (n.d.) History \& mission. Retrieved from http://www.omsi.edu/history-and-mission

Oregon Museum of Science and Industry (OMSI). (2017). Lenses on the Sky educator guide. Portland, OR: Oregon Museum of Science and Industry. Retrieved from https://omsi.edu/sites/default/files/LOTS\%20Educator\%20Guide\%20Intro.pdf

Osberg, D., \& Biesta, G. J. J. (2007). Beyond presence: Epistemological and pedagogical implications of "strong" emergence. Interchange: A Quarterly Review of Education, 38(1), 31-51. doi:10.1007/s10780-007-9014-3

Paris, D. (2012). Culturally sustaining pedagogy: A needed change in stance, terminology, and practice. Educational Researcher, 41(3), 93-97. doi:10.3102/0013189X12441244 
Paris, D. (2016). On educating culturally sustaining teachers. University of Michigan

TeachingWorks papers. Retrieved from http://www.teachingworks.org/images/files/TeachingWorks_Paris.pdf

Paris, D., \& Alim, H. S. (2014). What are we seeking to sustain through culturally sustaining pedagogy? A loving critique forward. Harvard Educational Review, 84(1), 85-100.

Paris, D., \& Ball, A. (2009). Teacher knowledge in culturally and linguistically complex classrooms: Lessons from the golden age and beyond. In L. M. Morrow, R. Rueda, \& D. Lapp (Eds.), Handbook of research on literacy instruction: Issues of diversity, policy, and equity (pp. 379-395). New York, NY: Guilford Press.

Parker, P. D., Marsh, H. W., Ciarrochi, J., Marshall, S., \& Abduljabbar, A. S. (2014). Juxtaposing math self-efficacy and self-concept as predictors of long-term achievement outcomes. Educational Psychology, 34(1), 29-48. doi:10.1080/01443410.2013.797339

Pattison, S., Gontan, I., Ramos-Montañez, S., Shagott, T., Francisco, M., \& Dierking, L. (2020). The identity-frame model: A framework to describe situated identity negotiation for adolescent youth participating in an informal engineering education program. Journal of the Learning Sciences, 29(4), 550-597. doi:10.1080/10508406.2020.1770762

Peralta, C., Caspary, M., \& Boothe, D. (2013). Success factors impacting Latina/o persistence in higher education leading to STEM opportunities. Cultural Studies of Science Education, 8(4), 905-918. doi:10.1007/s11422-013-9520-9 
Peters, M. (2005). Critical pedagogy and the futures of critical theory. In Ilan-Gur-Ze'ev (Ed.), Critical theory and critical pedagogy today: Toward a new critical language in education (pp. 35-38). Haifa: Faculty of Education, University of Haifa.

Phillips, D. C. (1990). Postpositivistic science: Myths and realities. In E. G. Guba (Ed), The paradigm dialog (pp. 31-45). Newbury Park, CA: SAGE.

Pintrich, P. R., Smith, D. A. F., Garcia, T., \& McKeachie, W. J. (1993). Reliability and predictive validity of the Motivated Strategies for Learning Questionnaire (MSLQ). Educational and Psychological Measurement, 53(3), 801-813. doi:10.1177/0013164493053003024

Portland State University. (2014). Graduate school of education doctor of education in educational leadership final dissertation paper rubric: 5-part dissertation. Retrieved from https://www.pdx.edu/education/sites/www.pdx.edu.education/files/doctoraldissertation-rubric.pdf

Price, G. (2017). Trump's budget proposal eliminates funding for IMLS, NEA, NEH, CPB, and more. Retrieved from http://www.infodocket.com/2017/03/16/trumpsfederal-budget-proposal-eliminates-funding-for-imls-nea-neh-cpb-and-more

Puzio, K., Newcomer, S., Pratt, K., McNeely, K., Jacobs, M., \& Hooker, S. (2017). Creative failures in culturally sustaining pedagogy. Language Arts, 94(4), 223233.

Ramos, B. M. (2012). Psychosocial stress, social inequality, and mental health in Puerto Rican women in upstate New York. Centro Journal, 249(2), 48-67. 
Ravn, I. (2016). Transformative theory in social and organizational research. World Futures, 72(7-8), 327-341. doi:10.1080/02604027.2014.989782

Reid, K. W. (2013). Understanding the relationships among racial identity, self-efficacy, institutional integration and academic achievement of Black males attending research universities. The Journal of Negro Education, 82(1), 75-93.

Rhodes, C. M. (2017). A validation study of the culturally responsive teaching survey. Universal Journal of Educational Research, 5(1), 45-53. doi:10.13189/ujer.2017.050106

Ricca, B. (2012). Beyond teaching methods: A complexity approach. Complicity: An International Journal of Complexity and Education, 9(2), 31-51.

Riedinger, K. (2012). Family conversations in informal learning environments. Childhood Education, 88(2), 125-127.

Robinson, V. M. J. (1994).The practical promise of critical research in educational administration. Educational Administration Quarterly, 30(1), 56-76. doi:10.1177/0013161X94030001005

Roots of Wisdom Project Team. (2016). Reflections and ideas about collaboration with integrity. Portland, OR: Oregon Museum of Science and Industry. Retrieved from https://omsi.edu/exhibitions/row/docs/ROW_LegacyDoc.pdf

Ryan, G. S. (2018). Introduction to positivism, interpretivism and critical theory. Nurse Researcher, 25(4), 13-19.

Ryan, J. (1998). Critical leadership for education in a postmodern world: Emancipation, resistance and communal action. International Journal of Leadership in Education, 1(3), 257-278. doi:10.1080/1360312980010303 
Sahin, A., Ekmekci, A., \& Waxman, H. C. (2017). The relationships among high school STEM learning experiences, expectations, and mathematics and science efficacy and the likelihood of majoring in STEM in college. International Journal of Science Education, 39(11), 1549-1572. doi:10.1080/09500693.2017.1341067

Sample McMeeking, L. S., Weinberg, A. E., Boyd, K. J., \& Balgopal, M. M. (2016). Student perceptions of interest, learning, and engagement from an informal traveling science museum. School Science and Mathematics, 116(5), 253 - 264. doi:10.1111/ssm.12176

Savahl, S., Adams, S., Isaacs, S., September, R., Hendricks, G., \& Noordien, Z. (2015). Subjective well-being amongst a sample of South African children: A descriptive study. Child Indicators Research, 8(1), 211-226.

Sayman, D. M. (2013). Quinceañeras and quadratics: Experiences of Latinas in statesupported residential schools of science and math. Journal of Latinos and Education, 12(3), 215-230. doi:10.1080/15348431.2013.765805

Schwarzer, R., \& Jerusalem, M. (1995). Generalized self-efficacy scale. In J. Weinman, S. Wright, \& M. Johnston (Eds.), Measures in health psychology: A user's portfolio. Causal and control beliefs (pp. 35-37). Windsor, UK: NFER-NELSON.

Sherer, M., Maddux, J. E., Mercandante, B., Prentice-Dunn, S., Jacobs, B., \& Rogers, R. W. (1982). The self-efficacy scale: Construction and validation. Psychological Reports, 51(2), 663-671. doi:10.2466/pr0.1982.51.2.663

Shein, P. P., Falk, J. H., \& Li, Y.-Y. (2019). The role of science identity in science center visits and effects. Science Education, 103(6), 1478-1492. doi:10.1002/sce.21535 
Siwatu, K. O. (2007). Preservice teachers' culturally responsive teaching self-efficacy and outcome expectancy beliefs. Teaching and Teacher Education, 23(7), 10861101. doi:10.1016/j.tate.2006.07.011

Southern California Grantmakers. (2016). The STEM funders network: First year findings of 27 learning ecosystems. Retrieved from https://www.socalgrantmakers.org/events/stem-funders-network-first-yearfindings-27-learning-ecosystems

Southern Education Foundation. (2016). Race and ethnicity in a new era of public funding of private schools: Private school enrollment in the south and the nation. Retrieved from http://www.southerneducation.org/getattachment/be785c57-6ce74682-b80d-04d89994a0b6/Race-and-Ethnicity-in-a-New-Era-of-PublicFunding.aspx

Steele, C. M., \& Aronson, J. (1995). Stereotype threat and the intellectual test performance of African Americans. Journal of Personality and Social Psychology, 69(5), 797-811.

STEM Funders Network. (2016). STEM ecosystems: Design principles. Retrieved from http://stemecosystems.org/design-principles/

Tang, M., Pan, W., \& Newmeyer, M. D. (2008). Factors influencing high school students' career aspirations. Professional School Counseling, 11(5), 285-295.

Taylor, E. (2016). Critical race theory and interest convergence in the backlash against affirmative action: Washington State and Initiative 200. In E. Taylor, D. Gillborn, \& G. Ladson-Billings (Eds), Foundations of critical race theory in education (pp. 113-122). New York, NY: Routledge. 
Taylor, S. (1997). Critical policy analysis: Exploring contexts, texts and consequences. Discourse: Studies in the Cultural Politics of Education, 18(1), 23-35.

Tavakol, M., \& Dennick, R. (2011). Making sense of Cronbach's alpha. International Journal of Medical Education, 2, 52-55. doi:10.5116/ijme.4dfb.8dfd

Teclaw, R., Price, M. C., \& Osatuke, K. (2012). Demographic question placement: Effect on item response rates and means of a Veterans Health Administration survey. Journal of Business and Psychology, 27(3), 281-290. doi:10.1007/s10869-0119249-y

Thom, M. (2001). Balancing the equation: Where are women and girls in science, engineering and technology. New York, NY: The National Council for Research on Women.

Tolia-Kelly, D. P. (2016). Feeling and being at the (postcolonial) museum: Presencing the affective politics of 'race' and culture. Sociology, 50(5), 896-912. doi:10.1177/0038038516649554

Tolliver, D. E. (2015). Africentrism-Standing on its own cultural ground. New Directions for Adult and Continuing Education, 2015(147), 59-70. doi:10.1002/ace.20142

Tunnicliffe, S. D. (2008). Conversations of family and primary school groups at robotic dinosaurs in a museum? What do they talk about? Journal of Elementary Science Education, 20(3), 17-33. doi:10.1007/BF03174706

Tushnet, M. V. (2016). The "we've done enough" theory of school desegregation. In E. Taylor, D. Gillborn, \& G. Ladson-Billings (Eds.), Foundations of critical race theory in education ( $2^{\text {nd }}$ ed., pp. 97-105). New York, NY: Routledge. 
Van Nuland, S. E., Hall, E., \& Langley, N. R. (2020). STEM crisis teaching: Curriculum design with e-learning tools. FASEB BioAdvances, 2(11), 631 - 637. doi:10.1096/fba.2020-00049

Varelas, M., Kane, J. M., \& Wylie, C. D. (2012). Young Black children and science: Chronotopes of narratives around their science journals. Journal of Research in Science Teaching, 49(5), 568-596. doi:10.1002/tea.21013

Varelas, M., Tucker-Raymond, E., \& Richards, K. (2015). A structure-agency perspective on young children's engagement in school science: Carlos's performance and narrative. Journal of Research in Science Teaching, 52(4), 516529. doi:10.1002/tea.21211

Vedder-Weiss, D., \& Fortus, D. (2011). Adolescents' declining motivation to learn science: Inevitable or not? Journal of Research in Science Teaching, 48(2), 199216.

Visser, P. S., Krosnick, J. A., \& Lavrakas, P. J. (2000). Survey research. In H. T. Reis \& C. M. Judd (Eds.), Handbook of research methods in social and personality psychology (pp. 223-252). New York, NY: Cambridge University Press.

Walsh, D. (2018). Youth participatory action research as culturally sustaining pedagogy. Theory Into Practice. doi:10.1080/00405841.2018.1433939

Walther, B., Cardiel, C., \& Reyes, N. (2014). Evaluation summary report for $\$ 2$ Days 2013-2014. Unpublished internal document, Oregon Museum of Science and Industry, Portland, OR. 
Wang, M-T., \& Degol, J. L. (2017). Gender gap in science, technology, engineering, and mathematics (STEM): Current knowledge, implications for practice, policy, and future directions. Educational Psychology Review, 29(1), 119-140. doi:10.1007/s10648-015-9355-X

Wang, X. (2013). Why students choose STEM majors: Motivation, high school learning, and postsecondary context of support. American Educational Research Journal, 50(5), 1081-1121. doi:10.3102/0002831213488622

Weiland, I. (2015). An exploration of Hispanic mothers' culturally sustaining experiences at an informal science center. Journal of Research in Science Teaching, 52(1), 84-106. doi:10.1002/tea.21190

Weis, L., Eisenhart, M., Cipollone, K., Stich, A. E., Nikischer, A. B.,...Dominguez, R. (2015). In the guise of STEM education reform: Opportunity structures and outcomes in inclusive STEM-focused high schools. American Educational Research Journal, 52(6), 1024-1059. doi:10.3102/0002831215604045

Whitaker, M. C., \& Valtierra, K. M. (2018). The dispositions for culturally responsive pedagogy scale. Journal for Multicultural Education, 12(1), 10-24. doi:10.1108/JME-11-2016-0060

Wolters, C. A. (2004). Advancing achievement goal theory: Using goal structures and goal orientations to predict students' motivation, cognition, and achievement. Journal of Educational Psychology, 96(2), 236-250. doi:10.1037/00220663.96 .2 .236 
Wood, P., \& Butt, G. (2014). Exploring the use of complexity theory and action research as frameworks for curriculum change. Journal of Curriculum Studies, 46(5), 676696. doi:10.1080/00220272.2014.921840

Yong, E. (2018). Opening plenary session and keynote presentation [Keynote presentation]. Association of Science-Technology Centers 2018 Annual Conference, Hartford, CT.

Young, M. D., \& Diem, S. (2014). Putting critical theoretical perspectives to work in educational policy. International Journal of Qualitative Studies in Education, 27(9), 1063-1067. doi:10.1080/09518398.2014.916015

Zellermayer, M., \& Margolin, I. (2005). Teacher educators’ professional learning described through the lens of complexity theory. Teachers College Record, 107(6), 1275-1304.

Zimmerman, B. J., Bandura, A., \& Martinez-Pons, M. (1992). Self-motivation for academic attainment: The role of self-efficacy beliefs and personal goal setting. American Educational Research Journal, 29(3), 663-676. doi:10.3102/00028312029003663 
Appendix A:

\section{Parent/Guardian Informed Consent Letter}

\section{蛋 Portland State}

What is this all about?

Thank you for considening this request for your child to participate in my study of leaning in and out of school! My name is Chris Cardiel, and this research is part of my doctoral work in the Portland State University Graduate School of Education under the instruction of Dr. Anita Bright.

All students in your child's science class are invited to participate in a survey to leam about Portland-area students' perceptions and experiences regarding science learning in and out of school. This survey will be available in Spanish and English and will be handed out during an upcoming class period at your child's school, during which I will be present to answer any questions. School and school district administrators have approved this survey, but if your child wishes to participate, their participation requires your consent.

What are the risks?

There are no major risks associated with participation in this study. I will know the school from which each survey is collected, but your child's name and other identifying information will not be included and their responses will be anonymous and will only be analyzed along with the responses of other students at their school, as well as students at other Portland-area schools.

What are the benefits?

Because the survey process requires classroom time, I am offering each participating class a free field trip to the Oregon Museum of Science and Industry as thanks to the students for taking the time to share their perspectives. Additionally, while (and after) completing the survey, your child may have a chance to think in new ways about how they connect with science topics in and out of school. Once the study is completed, I will make available (a) the dissertation produced as a result of this study and (b) the overall aggregated survey responses for your child's school.

What are my rights?

Your consent for your child to participate in this study and your child's assent to participate are voluntary and you or your child may withdraw consent at any time with no repercussions. If you have any questions prior to, during, or after my visit to your child's classroom, please contact Chris Cardiel (ccardiel_apdx.edu, 360-909-7273) or Anita Bright (abrightapdx.edu, 503-7254797). If you have concerns about your or your child's rights as a research participant, please contact the PSU Institutional Review Board Office of Research Integrity at 1600 SW 4th Ave., Market Center Building, Ste. 620 Portland, OR 97201 or (503) 725-2227.

By signing below, you signify your understanding of the information provided above and your consent for your child to participate in the survey. (Again, you or your child may withdraw this consent at any time and for any reason.) 
Appendix B:

Survey Instrument (Spanish)

\section{Encuesta estudiantil STEM Ecosystems (ecosistemas de ciencia, tecnología, ingeniería y matemáticas)}

¡Hola! Mi nombre es Chris Cardiel. Me encanta tener la oportunidad de conectar contigo hoy e invitarte a compartir tus opiniones y experiencias sobre cómo y dónde aprendes sobre la ciencia, la tecnología, la ingeniería y las matemáticas (STEM, por sus siglas en inglés).

Este estudio es parte de mi doctorado en la universidad Portland State University (PSU), a través del Graduate School of Education (escuela de posgrado en educación) y bajo la instrucción de la Dra. Anita Bright, una profesora de PSU. Como parte de mi estudio, quiero invitarte a participar en una encuesta para saber qué percepciones y experiencias tienen los estudiantes de secundaria sobre el aprendizaje de la ciencia, la tecnología, la ingeniería y las matemáticas tanto dentro como fuera de la escuela. Como agradecimiento por tomarte el tiempo de contestar esta encuesta y compartir tus opiniones y perspectivas, le estoy ofreciendo a todos quienes completen la encuesta una entrada gratis para visitar el museo de ciencia e industria Oregon Museum of Science and Industry (OMSI).

Esta encuesta está disponible en inglés y en español, y puedes completarla en el idioma que quieras. Esta encuesta tomará entre quince y veinte minutos para completar. Tu participación no causará mayores riesgos o molestias, aparte de cualquier incomodidad emocional o psicológica que te pueda provocar contestar preguntas sobre tu opinión, percepción y experiencias relacionadas con la educación. A pesar de que sabré de qué clase vino cada encuesta, tus respuestas serán anónimas y tu nombre nunca será asociado con la encuesta que completaste.

En cuanto a beneficios, durante y después de completar la encuesta tendrás la oportunidad de pensar en nuevas formas de conectar con temas científicos tanto dentro como fuera de tu escuela. Una vez que finalice mi estudio, pondré a disposición lo que escriba como resultado de este estudio y el resumen de las respuestas de tu escuela en particular, por si quieres recibir una o ambas. Tu participación es completamente voluntaria y puedes dejar de participar en cualquier momento sin consecuencia alguna. Tu decisión de participar o no participar en esta encuesta no tendrá ningún impacto en tus calificaciones o rendimiento académico.

Si tienes preguntas (ahora o más adelante), por favor ponte en contacto conmigo (ccardiel@pdx.edu, 360-909-7273) o con Anita Bright (abright@pdx.edu, 503-725-4797). Nos encantaría hablar contigo. Si tienes alguna inquietud sobre tus derechos como participante en esta investigación, por favor ponte en contacto con PSU Institutional Review Board Office of Research Integrity (1600 SW 4th Ave., Market Center Building, Ste. 620 Portland, OR 97201, (503) 725-2227). Si estás de acuerdo y quieres participar en esta encuesta, por favor presiona "Next" a continuación. Si no deseas participar, simplemente cierra esta página.

¡Muchas gracias! 
Piensa en las veces que aprendes o haces cosas relacionadas con la ciencia, la tecnología o las matemáticas (que abajo solo llamo "ciencias") en casa, y selecciona la opción que identifica cuánto estás de acuerdo o en desacuerdo con cada afirmación.

\section{Muy de acuerdo}

(1)
Algo de acuerdo

\section{Algo en \\ desacuerdo (3)}

Muy en

desacuerdo (4)

Podré alcanzar la mayor parte de las metas que me he propuesto.

Cuando me enfrento a retos difíciles, estoy seguro/a que los podré completar.

En casi todos los casos, creo que puedo obtener los resultados que busco si son importantes para mí.

Creo que puedo alcanzar el éxito en casi todo lo que me proponga.

Podré superar mis desafíos con éxito.

Estoy seguro/a que puedo realizar diferentes funciones de manera eficaz.

Cuando me comparo con otras personas de mi edad, puedo hacer la mayoría de las cosas muy bien.

Incluso cuando las cosas se ponen difíciles, me puedo desempeñar bien.

Me interesan y emocionan temas e ideas de la ciencia y quiero aprender más.

Puedo entender, recordar y usar la información que aprendo sobre la ciencia.

Puedo probar las ideas científicas que tengo y usar lo que he aprendido para entender mejor el mundo.

Tengo la oportunidad de pensar en cómo la ciencia me permite ver el mundo.

Hago actividades de ciencia con otros usando lenguaje científico y herramientas científicas.

Considero que soy alguien con una facilidad de aprender ciencia y hacer cosas científicas.

Puedo aprender sobre la ciencia en formas que se conectan a mi cultura.

Mi voz es escuchada y respetada cuando es hora de decidir cómo aprender sobre la ciencia.

Mi éxito en la ciencia es evaluado de una manera que demuestra respeto y atención a mi

cultura.

Las cosas que leo o veo cuando aprendo sobre la ciencia utilizan ejemplos y un lenguaje que refleja y valora mi cultura y mis experiencias personales.

Al aprender sobre la ciencia, tengo que ignorar mi cultura si quiero tener éxito.

Aprecio que mi familia y mis amistades se involucren en mi aprendizaje sobre la ciencia si es de una manera apropiada y que me haga sentir cómodo/a.

Puedo ser quien soy sin sentirme avergonzado/a de mi cultura o que se burlen de mí.

Puedo aprender sobre la ciencia de una manera que me ayuda a entender cómo tener éxito en la cultura predominante.

Puedo aprender sobre la ciencia en el idioma que me es más cómodo.

Puedo aprender sobre la historia y los logros científicos de personas de mi cultura. 
A continuación, piensa en las veces que aprendes o haces cosas relacionadas con la ciencia, la tecnología o las matemáticas (que abajo solo llamo "ciencias") en la escuela, y selecciona la opción que identifica cuánto estás de acuerdo o en desacuerdo con cada afirmación.

Muy de acuerdo

(1)
Algo de acuerdo

(2)
Algo en

desacuerdo (3)
Muy en

desacuerdo (4)

Podré alcanzar la mayor parte de las metas que me he propuesto.

Cuando me enfrento a retos difíciles, estoy seguro/a que los podré completar.

En casi todos los casos, creo que puedo obtener los resultados que busco si son importantes para mí.

Creo que puedo alcanzar el éxito en casi todo lo que me proponga.

Podré superar mis desafíos con éxito.

Estoy seguro/a que puedo realizar diferentes funciones de manera eficaz.

Cuando me comparo con otras personas de mi edad, puedo hacer la mayoría de las cosas muy bien.

Incluso cuando las cosas se ponen difíciles, me puedo desempeñar bien.

Me interesan y emocionan temas e ideas de la ciencia y quiero aprender más.

Puedo entender, recordar y usar la información que aprendo sobre la ciencia.

Puedo probar las ideas científicas que tengo y usar lo que he aprendido para entender mejor el mundo.

Tengo la oportunidad de pensar en cómo la ciencia me permite ver el mundo.

Hago actividades de ciencia con otros usando lenguaje científico y herramientas científicas.

Considero que soy alguien con una facilidad de aprender ciencia y hacer cosas científicas.

Puedo aprender sobre la ciencia en formas que se conectan a mi cultura.

Mi voz es escuchada y respetada cuando es hora de decidir cómo aprender sobre la ciencia.

Mi éxito en la ciencia es evaluado de una manera que demuestra respeto y atención a mi

cultura.

Las cosas que leo o veo cuando aprendo sobre la ciencia utilizan ejemplos y un lenguaje que refleja y valora mi cultura y mis experiencias personales.

Al aprender sobre la ciencia, tengo que ignorar mi cultura si quiero tener éxito.

Aprecio que mi familia y mis amistades se involucren en mi aprendizaje sobre la ciencia si es de una manera apropiada y que me haga sentir cómodo/a.

Puedo ser quien soy sin sentirme avergonzado/a de mi cultura o que se burlen de mí.

Puedo aprender sobre la ciencia de una manera que me ayuda a entender cómo tener éxito en la cultura predominante.

Puedo aprender sobre la ciencia en el idioma que me es más cómodo.

Puedo aprender sobre la historia y los logros científicos de personas de mi cultura. 
Ahora, piensa en las veces que aprendes o haces cosas relacionadas con la ciencia, la tecnología o las matemáticas (que abajo solo llamo "ciencias") en museos o centro de ciencia, y selecciona la opción que identifica cuánto estás de acuerdo o en desacuerdo con cada afirmación.

Muy de acuerdo

(1)
Algo de acuerdo
Algo en

desacuerdo (3)
Muy en desacuerdo (4)

Podré alcanzar la mayor parte de las metas que me he propuesto.

Cuando me enfrento a retos difíciles, estoy seguro/a que los podré completar.

En casi todos los casos, creo que puedo obtener los resultados que busco si son importantes para mí.

Creo que puedo alcanzar el éxito en casi todo lo que me proponga.

Podré superar mis desafíos con éxito.

Estoy seguro/a que puedo realizar diferentes funciones de manera eficaz.

Cuando me comparo con otras personas de mi edad, puedo hacer la mayoría de las cosas muy bien.

Incluso cuando las cosas se ponen difíciles, me puedo desempeñar bien.

Me interesan y emocionan temas e ideas de la ciencia y quiero aprender más.

Puedo entender, recordar y usar la información que aprendo sobre la ciencia.

Puedo probar las ideas científicas que tengo y usar lo que he aprendido para entender mejor el mundo.

Tengo la oportunidad de pensar en cómo la ciencia me permite ver el mundo.

Hago actividades de ciencia con otros usando lenguaje científico y herramientas científicas.

Considero que soy alguien con una facilidad de aprender ciencia y hacer cosas científicas.

Puedo aprender sobre la ciencia en formas que se conectan a mi cultura.

Mi voz es escuchada y respetada cuando es hora de decidir cómo aprender sobre la ciencia.

Mi éxito en la ciencia es evaluado de una manera que demuestra respeto y atención a mi

cultura.

Las cosas que leo o veo cuando aprendo sobre la ciencia utilizan ejemplos y un lenguaje que

refleja y valora mi cultura y mis experiencias personales.

Al aprender sobre la ciencia, tengo que ignorar mi cultura si quiero tener éxito.

Aprecio que mi familia y mis amistades se involucren en mi aprendizaje sobre la ciencia si es de una manera apropiada y que me haga sentir cómodo/a.

Puedo ser quien soy sin sentirme avergonzado/a de mi cultura o que se burlen de mí.

Puedo aprender sobre la ciencia de una manera que me ayuda a entender cómo tener éxito en la cultura predominante.

Puedo aprender sobre la ciencia en el idioma que me es más cómodo.

Puedo aprender sobre la historia y los logros científicos de personas de mi cultura. 
Finalmente, piensa en las veces que aprendes o haces cosas relacionadas con la ciencia, la tecnología o las matemáticas (que abajo solo llamo "ciencias") en otros lugares fuera de la escuela, como parques, centros comunitarios, etc., y selecciona la opción que identifica cuánto estás de acuerdo o en desacuerdo con cada afirmación.

Muy de acuerdo

(1)
Algo de acuerdo
Algo en

desacuerdo (3)
Muy en desacuerd o (4)

Podré alcanzar la mayor parte de las metas que me he propuesto.

Cuando me enfrento a retos difíciles, estoy seguro/a que los podré completar.

En casi todos los casos, creo que puedo obtener los resultados que busco si son importantes para mí.

Creo que puedo alcanzar el éxito en casi todo lo que me proponga.

Podré superar mis desafíos con éxito.

Estoy seguro/a que puedo realizar diferentes funciones de manera eficaz.

Cuando me comparo con otras personas de mi edad, puedo hacer la mayoría de las cosas muy bien.

Incluso cuando las cosas se ponen difíciles, me puedo desempeñar bien.

Me interesan y emocionan temas e ideas de la ciencia y quiero aprender más.

Puedo entender, recordar y usar la información que aprendo sobre la ciencia.

Puedo probar las ideas científicas que tengo y usar lo que he aprendido para entender mejor el mundo.

Tengo la oportunidad de pensar en cómo la ciencia me permite ver el mundo.

Hago actividades de ciencia con otros usando lenguaje científico y herramientas científicas. Considero que soy alguien con una facilidad de aprender ciencia y hacer cosas científicas.

Puedo aprender sobre la ciencia en formas que se conectan a mi cultura.

Mi voz es escuchada y respetada cuando es hora de decidir cómo aprender sobre la ciencia.

Mi éxito en la ciencia es evaluado de una manera que demuestra respeto y atención a mi cultura.

Las cosas que leo o veo cuando aprendo sobre la ciencia utilizan ejemplos y un lenguaje que refleja y valora mi cultura y mis experiencias personales.

Al aprender sobre la ciencia, tengo que ignorar mi cultura si quiero tener éxito.

Aprecio que mi familia y mis amistades se involucren en mi aprendizaje sobre la ciencia si es de una manera apropiada y que me haga sentir cómodo/a.

Puedo ser quien soy sin sentirme avergonzado/a de mi cultura o que se burlen de mí.

Puedo aprender sobre la ciencia de una manera que me ayuda a entender cómo tener éxito en la cultura predominante.

Puedo aprender sobre la ciencia en el idioma que me es más cómodo.

Puedo aprender sobre la historia y los logros científicos de personas de mi cultura. 
¿Con qué género te identificas?

¿Con qué grupo racial te identificas? (Por favor marca todos los que sean necesarios)

Indígena americano o nativo de Alaska Negro o afroamericano Blanco

Asiático o asiático americano

Nativo de Hawai o isleño del Pacífico

Prefiero no decir

Otro (por favor detallar)

¿Te consideras parte de alguno de estos grupos étnicos y culturales? (Por favor marca todos los que sean necesarios)

Colombiano/a Cubano/a Guatemalteco/a Hondureño/a Mexicano/a

Salvadoreño/a

Asiático/a de India Chino/a Filipino/a Japonés/a Coreano/a Vietnamita

Hawaiano/a Guameño/a Chamorro/a Samoano/a Otro (por favor detallar)

Ninguno de los anteriores Prefiero no decir

Si quieres recibir una entrada de admisión general a OMSI como agradecimiento por participar en esta encuesta, por favor escribe tu nombre y correo electrónico aquí. Cabe señalar que esta información NO será asociada con tus respuestas en la encuesta y SÓLO se usará para enviarte tu entrada a OMSI.

Muchas gracias por tu tiempo y tu contribución a este estudio. ¡Se te agradece profundamente! 
Appendix C:

Survey Instrument (English)

\section{STEM Ecosystems Student Survey}

Hello! My name Chris Cardiel, and I am so excited to connect with you today to invite you to share your thoughts and experiences regarding how and where you learn about science, technology, engineering, and math (STEM).

This research is part of my doctoral work at Portland State University through the Graduate School of Education under the instruction of Dr. Anita Bright, one of the professors at PSU. As part of my study, I want to invite you to participate in a survey to learn about high school students' perceptions and experiences regarding (STEM) learning in and out of school. Because I am taking some of your time to ask you to complete this survey, I am offering everyone who completes the survey a ticket good for a free visit to the Oregon Museum of Science and Industry (OMSI) as thanks for taking the time to share your thoughts and perspectives.

This survey is available in Spanish and English, and you are welcome to use whichever version you would like. The survey should take between fifteen and twenty minutes to complete, and aside from the minimal risk of psychological or emotional discomfort that might result from taking a survey asking about educational opinions, perceptions, and experiences, there are no major risks or discomforts connected to participating. While I will know which class each survey came from, your responses will be anonymous and your name will never be connected with the survey you complete.

In terms of benefits, during and after your time completing the survey, you may have a chance to think in new ways about how you connect with science topics in and out of school. Once my study is completed, I will make available both the paper I write as a result of this study and the overall survey responses for your school, you would like to receive either or both. Your choice to participate is completely voluntary and you can change your mind at any time with no negative consequences. Also, your choice about whether or not to participate will have no impact on your grades or academic standing in your class.

If you have any questions, now or later, please contact me (ccardiel@pdx.edu, 360-909-7273) or Anita Bright (abright@pdx.edu, 503-725-4797) and we will be happy to chat with you. If you have concerns about your rights as a research participant, please contact the PSU Institutional Review Board Office of Research Integrity (1600 SW 4th Ave., Market Center Building, Ste. 620 Portland, OR 97201, (503) 725-2227). If you agree and would like to participate in this survey, please click the "Next" button below, otherwise please just close this page.

Thank you so much again! 
Think about times when you learn about or do things related to science, technology, or math (which I'm just calling "science" below") at home, then select the option that shows how much you agree or disagree with each statement.

$$
\text { Agree a lot (1) Agree slightly (2) } \begin{gathered}
\text { Disagree slightly } \\
\text { (3) }
\end{gathered} \quad \text { Disagree a lot (4) }
$$

I will be able to achieve most of the goals that I have set for myself.

When facing difficult tasks, I am certain that I will complete them.

In most cases, I think that I can get the results I want if they are important to me.

I believe I can succeed at most anything I put my mind to.

I will be able to successfully overcome many challenges.

I am confident that I can perform effectively on many different tasks.

Compared to other people my age, I can do most tasks very well.

Even when things are tough, I can perform quite well.

I feel excited about and interested in science topics or ideas and want to learn more about them.

I am able to understand, remember, and use the information I learn about science.

I can test the science ideas I have and use what I learn to better understand the world.

I am given a chance to think about how science lets me see the world.

I do science activities with others using science language and tools.

I think of myself as someone who is good at learning and doing science-related things.

I can learn about science in ways that connect to my cultural heritage.

My voice is heard and respected in deciding how I learn about science.

My success in science is judged in ways that show respect and caring for my culture.

The things I read or watch when I learn about science use language and examples that reflect and value my culture and personal experiences.

I need to turn my back on my cultural heritage if I want to be successful at learning about science.

My family and friends are welcomed to be involved in my science learning in ways that feel comfortable and appropriate to me.

I am able to be myself without being made fun of or ashamed of my cultural heritage.

I can learn about science in ways that help me understand how to succeed in mainstream culture.

I can learn about science in the language that is most comfortable for me.

I am able to learn about the science history and accomplishments of people from my culture. 
Next, think about times when you learn about or do things related to science, technology, or math (which I'm just calling "science" below") at school, then select the option that shows how much you agree or disagree with each statement.

$$
\text { Agree a lot (1) Agree slightly (2) } \begin{array}{ccc}
\text { Disagree slightly } & \text { (3) }
\end{array} \text { Disagree a lot (4) }
$$

I will be able to achieve most of the goals that I have set for myself.

When facing difficult tasks, I am certain that I will complete them.

In most cases, I think that I can get the results I want if they are important to me.

I believe I can succeed at most anything I put my mind to.

I will be able to successfully overcome many challenges.

I am confident that I can perform effectively on many different tasks.

Compared to other people my age, I can do most tasks very well.

Even when things are tough, I can perform quite well.

I feel excited about and interested in science topics or ideas and want to learn more about them.

I am able to understand, remember, and use the information I learn about science.

I can test the science ideas I have and use what I learn to better understand the world.

I am given a chance to think about how science lets me see the world.

I do science activities with others using science language and tools.

I think of myself as someone who is good at learning and doing science-related things.

I can learn about science in ways that connect to my cultural heritage.

My voice is heard and respected in deciding how I learn about science.

My success in science is judged in ways that show respect and caring for my culture.

The things I read or watch when I learn about science use language and examples that reflect and value my culture and personal experiences.

I need to turn my back on my cultural heritage if I want to be successful at learning about science.

My family and friends are welcomed to be involved in my science learning in ways that feel comfortable and appropriate to me.

I am able to be myself without being made fun of or ashamed of my cultural heritage.

I can learn about science in ways that help me understand how to succeed in mainstream culture.

I can learn about science in the language that is most comfortable for me.

I am able to learn about the science history and accomplishments of people from my culture. 
Now, think about times when you learn about or do things related to science, technology, or math (which I'm just calling "science" below") at science centers or museums, then select the option that shows how much you agree or disagree with each statement.
Agree a lot (1)
Agree slightly (2)
Disagree slightly
Disagree a lot (4)

I will be able to achieve most of the goals that I have set for myself.

When facing difficult tasks, I am certain that I will complete them.

In most cases, I think that I can get the results I want if they are important to me.

I believe I can succeed at most anything I put my mind to.

I will be able to successfully overcome many challenges.

I am confident that I can perform effectively on many different tasks.

Compared to other people my age, I can do most tasks very well.

Even when things are tough, I can perform quite well.

I feel excited about and interested in science topics or ideas and want to learn more about them.

I am able to understand, remember, and use the information I learn about science.

I can test the science ideas I have and use what I learn to better understand the world.

I am given a chance to think about how science lets me see the world.

I do science activities with others using science language and tools.

I think of myself as someone who is good at learning and doing science-related things.

I can learn about science in ways that connect to my cultural heritage.

My voice is heard and respected in deciding how I learn about science.

My success in science is judged in ways that show respect and caring for my culture.

The things I read or watch when I learn about science use language and examples that reflect and value my culture and personal experiences.

I need to turn my back on my cultural heritage if I want to be successful at learning about science.

My family and friends are welcomed to be involved in my science learning in ways that feel comfortable and appropriate to me.

I am able to be myself without being made fun of or ashamed of my cultural heritage.

I can learn about science in ways that help me understand how to succeed in mainstream culture.

I can learn about science in the language that is most comfortable for me.

I am able to learn about the science history and accomplishments of people from my culture. Lastly, think about times when you learn about or do things related to science, technology, or math (which I'm just calling "science" below") in other places outside of school, like parks, 
A MULTIPLICITY OF JOURNEYS

community centers, etc., then select the option that shows how much you agree or disagree with each statement.

$$
\text { Agree a lot (1) Agree slightly (2) } \quad \begin{gathered}
\text { Disagree slightly } \\
(3)
\end{gathered} \quad \text { Disagree a lot (4) }
$$

I will be able to achieve most of the goals that I have set for myself.

When facing difficult tasks, I am certain that I will complete them.

In most cases, I think that I can get the results I want if they are important to me.

I believe I can succeed at most anything I put my mind to.

I will be able to successfully overcome many challenges.

I am confident that I can perform effectively on many different tasks.

Compared to other people my age, I can do most tasks very well.

Even when things are tough, I can perform quite well.

I feel excited about and interested in science topics or ideas and want to learn more about them.

I am able to understand, remember, and use the information I learn about science.

I can test the science ideas I have and use what I learn to better understand the world.

I am given a chance to think about how science lets me see the world.

I do science activities with others using science language and tools.

I think of myself as someone who is good at learning and doing science-related things.

I can learn about science in ways that connect to my cultural heritage.

My voice is heard and respected in deciding how I learn about science.

My success in science is judged in ways that show respect and caring for my culture.

The things I read or watch when I learn about science use language and examples that reflect and value my culture and personal experiences.

I need to turn my back on my cultural heritage if I want to be successful at learning about science.

My family and friends are welcomed to be involved in my science learning in ways that feel comfortable and appropriate to me.

I am able to be myself without being made fun of or ashamed of my cultural heritage.

I can learn about science in ways that help me understand how to succeed in mainstream culture.

I can learn about science in the language that is most comfortable for me.

I am able to learn about the science history and accomplishments of people from my culture. 
What is your gender?

How do you identify in terms of race? (Please select all that apply.)

American Indian or Alaska Native Black or African-American White

Asian or Asian-American Native Hawaiian or Other Pacific Islander

Prefer not to say Other (please describe)

Do you consider yourself part of these ethnic/cultural groups? (Please select as many as you like.)

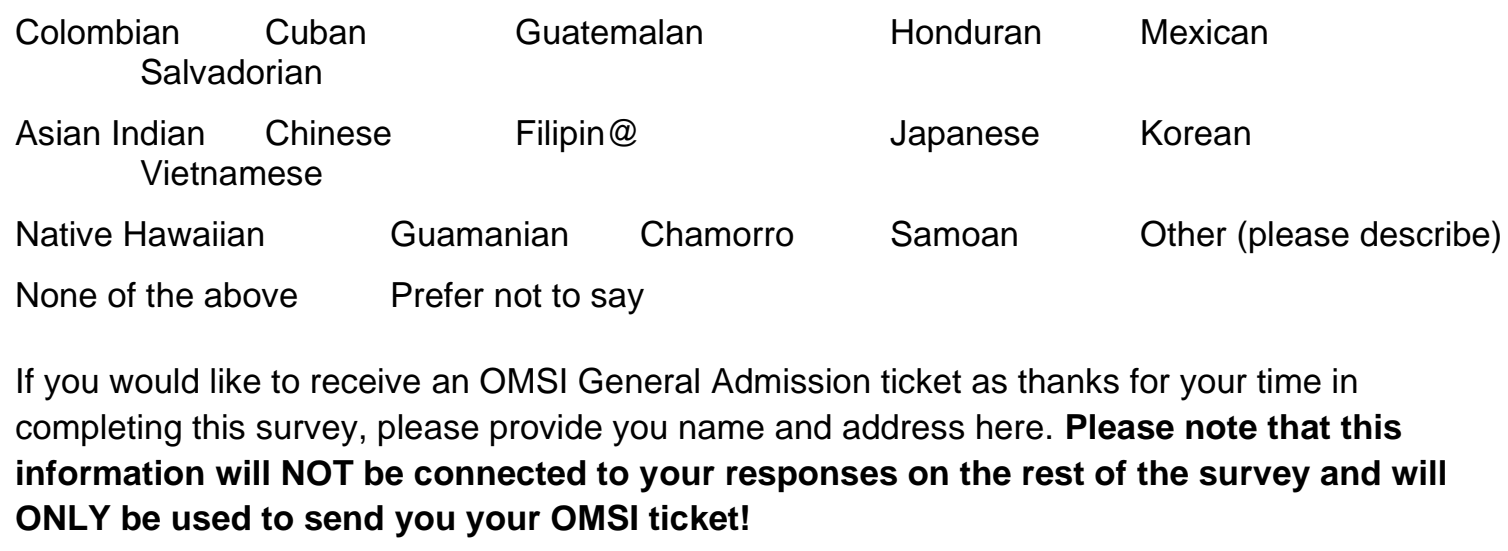

Thank you so much for your time and contributions to this study--it is deeply appreciated! 
Appendix D:

\section{Established Self-Efficacy Measures}

1. I will be able to achieve most of the goals that I have set for myself.

2. When facing difficult tasks, I am certain that I will accomplish them.

3. In general, I think that I can obtain outcomes that are important to me.

4. I believe I can succeed at most any endeavor to which I set my mind.

5. I will be able to successfully overcome many challenges.

6. I am confident that I can perform effectively on many different tasks.

7. Compared to other people, I can do most tasks very well.

8. Even when things are tough, I can perform quite well. General Self-Efficacy Scale (Chen, Gully, \& Eden, 2001)

1. I'm certain I can master the skills taught in class this year.

2. I'm certain I can figure out how to do the most difficult class work.

3. I can do almost all the work in class if I don't give up.

4. Even if the work is hard, I can learn it.

5. I can do even the hardest work in this class if I try. Academic Efficacy Scale (Midgley et al., 1993)

1. I believe I will receive an excellent grade in this class.

2. I'm certain I can understand the most difficult material presented in the readings for this course.

3. I'm confident I can understand the basic concepts taught in this course.

4. I'm confident I can understand the most complex material presented by the instructor in this course.

5. I'm confident I can do an excellent job on the assignments and tests in this course.

6. I expect to do well in this class.

7. I'm certain I can master the skills being taught in this class.

8. Considering the difficulty of this course, the teacher, and my skills, I think I will do well in this class.

Self-Efficacy for Learning and Performance Scale (Pintrich, Smith, Garcia, \& McKeachie, 1993)

1. I can always manage to solve difficult problems if I try hard enough.

2. If someone opposes me, I can find the means and ways to get what I want.

3. It is easy for me to stick to my aims and accomplish my goals.

4. I am confident that I could deal efficiently with unexpected events. 
5. Thanks to my resourcefulness, I know how to handle unforeseen situations.

6. I can solve most problems if I invest the necessary effort.

7. I can remain calm when facing difficulties because I can rely on my coping abilities.

8. When I am confronted with a problem, I can usually find several solutions.

9. If I am in trouble, I can usually think of a solution

10. I can usually handle whatever comes my way.

General Self-Efficacy Scale (Schwarzer \& Jerusalem, 1995)

1. When I make plans, I am certain I can make them work.

2. One of my problems is that I cannot get down to work when I should.

3. If I can't do a job the first time, I keep trying until I can.

4. When I set important goals for myself, I rarely achieve them.

5. I give up on things before completing them.

6. If something looks too complicated, I will not even both to try it.

7. When I have something unpleasant to do, I stick to it until I finish it.

8. When I decide to do something, I go right to work on it.

9. When trying to learn something new, I soon give up if I am not initially successful.

10. When unexpected problems occur, I don't handle them well.

11. I avoid trying to learn new things when they look too difficult.

12. Failure just makes me try harder.

13. I feel insecure about my ability to do things.

14. I am a self-reliant person.

15. I give up easily.

16. I do not seem capable of dealing with most problems that come up in life.

General Self-Efficacy Scale (Sherer, Maddux, Mercandante, Prentice-Dunn, Jacobs, \& Rogers, 1982)

\section{How well can you:}

\section{Self-efficacy for self-regulated learning}

1. finish homework assignments by deadlines?

2. study when there are other interesting things to do?

3. concentrate on school subjects?

4. take class notes of class instruction?

5. use the library to get information for class assignments?

6. plan your schoolwork?

7. organize your schoolwork?

8. remember information presented in class and textbooks? 
9. arrange a place to study without distractions?

10. motivate yourself to do schoolwork?

11. participate in class discussions?

\section{Self-efficacy for academic achievement}

1. learn general mathematics?

2. learn algebra?

3. learn science?

4. learn biology?

5. learn reading and writing language skills?

6. learn to use computers?

7. learn foreign languages?

8. learn social studies?

9. learn English grammar?

Academic Self-Efficacy Scales (Zimmerman, Bandura, \& Martinez-Pons, 1992) 
Appendix E:

Established Culturally Responsive Teaching Measures

\section{Indigenous cultural value}

1. Students specific cultural identities are valued in this classroom

2. I communicate personally with families

3. Resources with local Indigenous content are provided

4. Cultural values are verbally endorsed

5. Relatives and community Elders are invited to contribute to or observe classroom learning

6. Contemporary aboriginal and Torres Strait Islander perspectives are included in all subject areas

7. Local community has input into curriculum content and process

\section{Explicitness}

8. Individual scaffolding is provided to all students so each can perform required learning tasks

9. I ensure my explanations are succinct

10. The learning priorities of the classroom are made clear

11. Learning objectives are displayed and articulated

12. I give constructive individual feedback

13. The learning focus for lessons is orally communicated throughout lessons

\section{Self-regulation support}

14. Students are given time to think things through in their own minds

15. I use individual student's strengths to support individual and collective learning

16. Lessons are paced to allow students time for task completion

17. Students reflect on their goal achievement

18. Time is given for students to respond to questions or during discussion

19. Individual goals for student achievement are established

20. Students work together and help others on activities and problems

21. Students are given choices about work e.g. modes content timing order of tasks where to work

22. Students conduct self-assessments of work completed

23. I act as a learning facilitator

24. Students are provided with time to ensure mastery of ideas

\section{Ethic of care}

25. I ensure that students know that their success and value is not determined only by academic achievement

26. I have a warm respectful manner to all students 
27. I spend individual time with all students in matters pertaining to their learning

28. I communicate high academic expectations for students

29. I engage with all students in positive conversation in matters that display evidence of my interest in the student

30. I explicitly encourage learner development in the broad sense not just academic learning

31. I positively acknowledge all students verbally or non-verbally outside the classroom

32. Learning success is celebrated

33. I display positive gestures e.g. smiles towards all students

\section{Literacy teaching}

34. Buddy reading occurs

35. The vocabulary and language of each curriculum area are explicitly taught

36. Literacy skills are taught and practiced in the context of modelled age appropriate text

37. ESL strategies are used when teaching students learning English as a second or additional language

38. Basic literacy skills are regularly revised

39. I orientate students to the vocabulary background knowledge and features of a text before reading

40. Oral language is used to develop literacy competence in SAE

\section{Behaviour support}

41. Skills and behaviours are modelled for students

42. I address off task behaviour with less intrusive correction skills such as non verbal cues and proximity

43. Students are able to contribute to the setting of the behavioural expectations for the classroom

44. Routines provide students with foreknowledge of activities and expectations

45. Consequences for student behaviour are made clear

46. I communicate and follow through on expectations about expected classroom behaviour

47. I communicate high behavioural expectations for students

\section{Pedagogical expertise}

48. Many examples are provided to support students in their learning

49. Tasks carried out encourage student Creativity and independent thinking

50. I use multiple strategies to assist students in their learning

51. Intervention is provided for those students not achieving the expected attainment for their age cohort

52. Students show their learning in various ways not just written 
53. Learning and assessment are placed within the broader contexts of what is familiar to students

54. Learning Experiences that cater for a variety of learning preferences are provided

55. Learning is chunked into short teaching segments

56. Hands on experiential activities are provided to support learning

57. I model thinking processes aloud

58. Multiple methods are used to explain abstract ideas

59. Students are provided with many opportunities to master skills

60. Narrative and story are used across the content areas

61. Open ended learning activities are provided

62. Visual images are used to support understanding of ideas

Culturally Responsive Pedagogy Scale (Boon \& Lewthwaite, 2015)

\section{I am able to...}

1. infuse the curriculum and thematic units with the culture of students represented in the classroom.

2. review and assess curricula and instructional materials to determine their multicultural strengths and weakness, and relevance to students' interest and instructional needs, and revise them if necessary.

3. develop a repertoire of instructional examples that are culturally familiar to students to serve as a scaffold for learning.

4. find ways to support language acquisition and enhance culturally and linguistically diverse students' comprehension of classroom tasks.

5. use a variety of assessment techniques, such as self-assessment, portfolios, and so on, to evaluate students' performance in favor of cultural diversity.

6. design assessments to complement the culturally responsive pedagogical strategies that were employed during instruction.

7. assess culturally diverse students' readiness, intellectual and academic strengths and weaknesses, and development needs.

8. utilize a variety of instructional methods to match students' learning preferences in learning the subject matter, and maintaining their attention and interest in learning.

9. know how to communicate with culturally diverse students and their parents or guardians.

10. structure classroom-based meetings that are comfortable for parents.

11. foster meaningful and supportive relationships with parents and families, and actively involve them in their students' learning.

12. use non-traditional discourse styles with culturally diverse students in an attempt to communicate in culturally responsive ways. 
13. communicate expectations of success to culturally diverse students.

14. establish expectations for appropriate classroom behavior in considering students' cultural backgrounds to maintain a conducive learning environment.

15. develop and maintain positive, meaningful, caring, and trusting relationships with students.

16. create a warm, supporting, safe, and secure classroom environment for culturally diverse students.

17. create a community of learners by encouraging students to focus on collective work, responsibility, and cooperation.

18. provide students with knowledge and skills needed to function in mainstream culture.

Culturally Responsive Teacher Preparedness Scale (Hsiao, 2015)

1. I include lessons about the acculturation process.

2. Examine class materials for culturally appropriate images and themes

3. I ask students to compare their culture with American culture.

4. I make an effort to get to know my students' families and backgrounds.

5. I learn words in my students' native languages.

6. I use mixed-language and mixed-cultural pairings in group work.

7. I use peer tutors or student-led discussions.

8. I use surveys to find out about my students' classroom preferences.

9. I elicit students' experiences in pre-reading and pre-listening activities.

10. I encourage students to speak their native languages with their children.

11. I have students work independently, selecting their own learning activities.

12. I spend time outside of class learning about the cultures and languages of my students.

13. I include lessons about anti-immigrant discrimination or bias.

14. I supplement the curriculum with lessons about international events.

15. I ask for student input when planning lessons and activities.

16. I encourage students to use cross-cultural comparisons when analyzing material.

17. I provide rubrics and progress reports to students. Culturally Responsive Teaching Practices Survey (Rhodes, 2017)

1. A positive teacher-student relationship can be established by building a sense of trust in my students.

2. Incorporating a variety of teaching methods will help my students to be successful.

3. Students will be successful when instruction is adapted to meet their needs. 
4. Developing a community of learners when my class consists of students from diverse cultural backgrounds will promote positive interactions between students.

5. Acknowledging the ways that the school culture is different from my students' home culture will minimize the likelihood of discipline problems.

6. Understanding the communication preferences of my students will decrease the likelihood of student-teacher communication problems.

7. Connecting my students' prior knowledge with new incoming information will lead to deeper learning.

8. Matching instruction to the students' learning preferences will enhance their learning.

9. Revising instructional material to include a better representation of the students' cultural group will foster positive self-images.

10. Providing English Language Learners with visual aids will enhance their understanding of assignments.

11. Students will develop an appreciation for their culture when they are taught about the contributions their culture has made over time.

12. Conveying the message that parents are an important part of the classroom will increase parent participation.

13. The likelihood of student-teacher misunderstandings decreases when my students' cultural background is understood.

14. Changing the structure of the classroom so that it is compatible with my students' home culture will increase their motivation to come to class.

15. Establishing positive home-school relations will increase parental involvement.

16. Student attendance will increase when a personal relationship between the teacher and students has been developed.

17. Assessing student learning using a variety of assessment procedures will provide a better picture of what they have learned.

18. Using my students' interests when designing instruction will increase their motivation to learn.

19. Simplifying the language used during the presentation will enhance English Language Learners' comprehension of the lesson.

20. The frequency that students' abilities are misdiagnosed will decrease when their standardized test scores are interpreted with caution.

21. Encouraging students to use their native language will help to maintain students' cultural identity.

22. Students' self-esteem can be enhanced when their cultural background is valued by the teacher.

23. Helping students from diverse cultural backgrounds succeed in school will increase their confidence in their academic ability. 
24. Students' academic achievement will increase when they are provided with unbiased access to the necessary learning resources.

25. Using culturally familiar examples will make learning new concepts easier.

26. When students see themselves in the pictures that are displayed in the classroom, they develop a positive self-identity.

Culturally Responsive Teaching Outcome Expectations Scale (Siwatu, 2007)

1. Adapt instruction to meet the needs of my students

2. Obtain information about my students' academic strengths

3. Determine whether my students like to work alone or in a group

4. Determine whether my students feel comfortable competing with other students

5. Identify ways that the school culture (e.g., values, norms, and practices) is different from my students' home culture

6. Implement strategies to minimize the effects of the mismatch between my students' home culture and the school culture

7. Assess student learning using various types of assessments

8. Obtain information about my students' home life

9. Build a sense of trust in my students

10. Establish positive home-school relations

11. Use a variety of teaching methods

12. Develop a community of learners when my class consists of students from diverse backgrounds

13. Use my students' cultural background to help make learning meaningful

14. Use my students' prior knowledge to help them make sense of new information

15. Identify ways how students communicate at home may differ from the school norms

16. Obtain information about my students' cultural background

17. Teach students about their cultures' contributions to science

18. Greet English Language Learners with a phrase in their native language

19. Design a classroom environment using displays that reflects a variety of cultures

20. Develop a personal relationship with my students

21. Obtain information about my students' academic weaknesses

22. Praise English Language Learners for their accomplishments using a phrase in their native language

23. Identify ways that standardized tests may be biased towards linguistically diverse students

24. Communicate with parents regarding their child's educational progress

25. Structure parent-teacher conferences so that the meeting is not intimidating for parents 
26. Help students to develop positive relationships with their classmates

27. Revise instructional material to include a better representation of cultural groups

28. Critically examine the curriculum to determine whether it reinforces negative cultural stereotypes

29. Design a lesson that shows how other cultural groups have made use of mathematics

30. Model classroom tasks to enhance English Language Learners' understanding

31. Communicate with the parents of English Language Learners regarding their child's achievement

32. Help students feel like important members of the classroom

33. Identify ways that standardized tests may be biased towards culturally diverse students

34. Use a learning preference inventory to gather data about how my students like to learn

35. Use examples that are familiar to students from diverse cultural backgrounds

36. Explain new concepts using examples that are taken from my students' everyday lives

37. Obtain information regarding my students' academic interests

38. Use the interests of my students to make learning meaningful for them

39. Implement cooperative learning activities for those students who like to work in groups

40. Design instruction that matches my students' developmental needs Culturally Responsive Teaching Self-Efficacy Scale (Siwatu, 2007)

\section{Disposition for Praxis}

1. I value assessing my teaching practices.

2. I am open to feedback about my teaching practices.

3. I am aware of my cultural background.

4. I am willing to be vulnerable.

5. I am willing to examine my own identities.

6. I am willing to take advantage of professional development opportunities focused on issues of diversity.

\section{Disposition for Community}

7. I value collaborative learning.

8. I value collaborating with families.

9. I view myself as a member of the learning community along with students.

10. I value student input into classroom rules.

11. I value developing personal relationships with students.

12. I value dialog as a way to learn about students' out of school lives. 
13. I am comfortable with conflict as an inevitable part of the teaching and learning processes.

14. I value student differences.

15. I value collaborating with colleagues.

\section{Disposition for Social Justice}

16. I believe that hot topic conversations (e.g., race, gender, sexuality, religion, etc.) should be had in class when necessary and/or relevant.

17. I believe that schools can reproduce social inequities.

18. I believe it is important to acknowledge how issues of power are enacted in schools.

19. I value equity (giving each student what they individually need) over equality (giving each student the same thing).

Dispositions for Culturally Responsive Pedagogy Scale (Whitaker \& Valtierra, 2018) 
Appendix F:

\section{Culturally Sustaining STEM Learning Experiences Scale}

1. I can learn about science in ways that connect to my cultural heritage.

2. My voice is heard and respected in deciding how I learn about science.

3. My success in science is judged in ways that show respect and caring for my culture.

4. The things I read or watch when I learn about science use language and examples that reflect and value my culture and personal experiences.

5. I need to turn my back on my cultural heritage if I want to be successful at learning about science.*

6. My family and friends are welcomed to be involved in my science learning in ways that feel comfortable and appropriate to me.

7. I am able to be myself without being made fun of or ashamed of my cultural heritage.

8. I can learn about science in ways that help me understand how to succeed in mainstream culture.

9. I can learn about science in the language that is most comfortable for me.

10. I am able to learn about the science history and accomplishments of people from my culture.

*Note that Item 5 was removed from analyses due to negative impact on Cronbach's $\alpha$ 